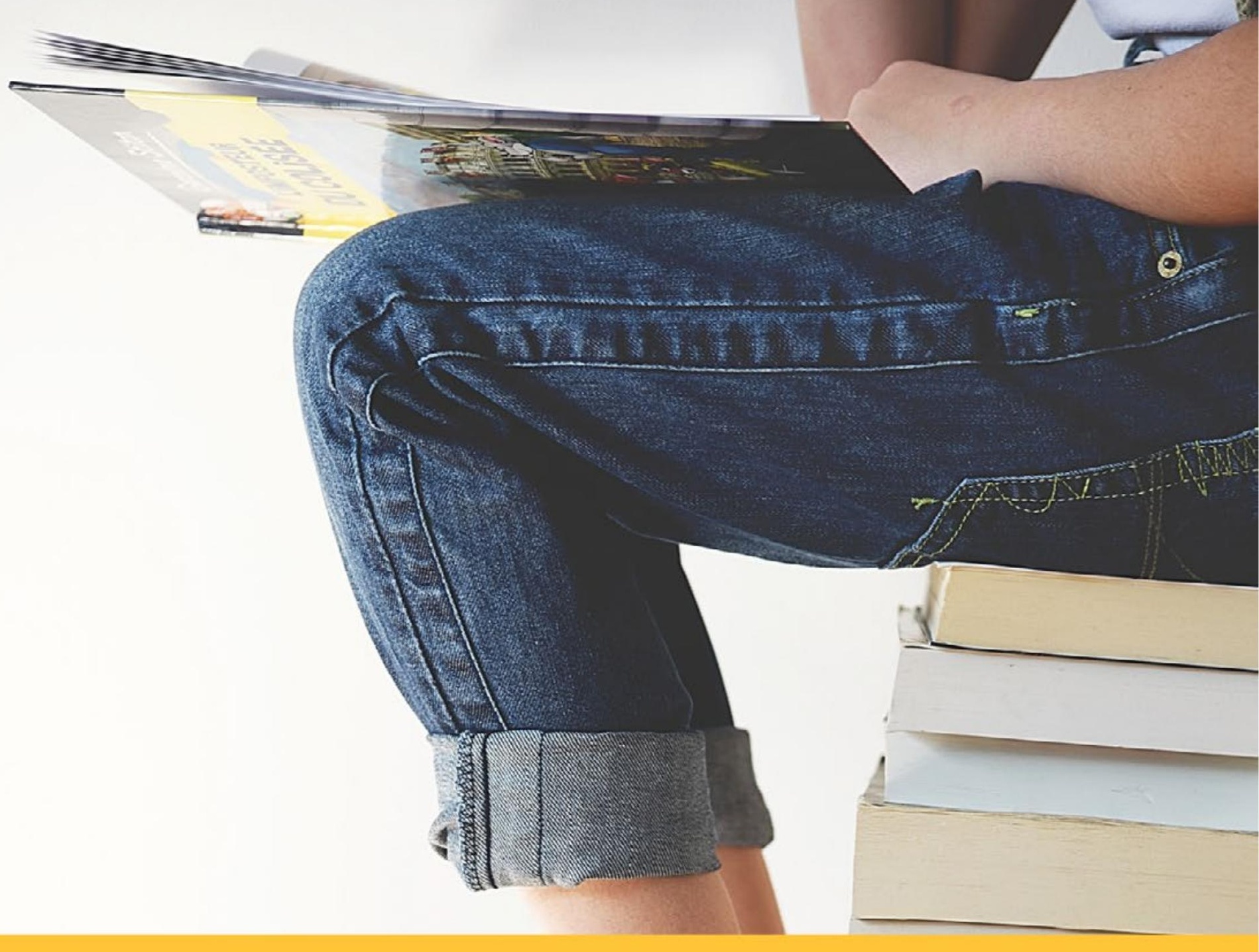

La recherche-action et la recherche-développement au service de la littératie

Sous la direction d'Isabelle Carignan, Marie-Christine Beaudry et François Larose

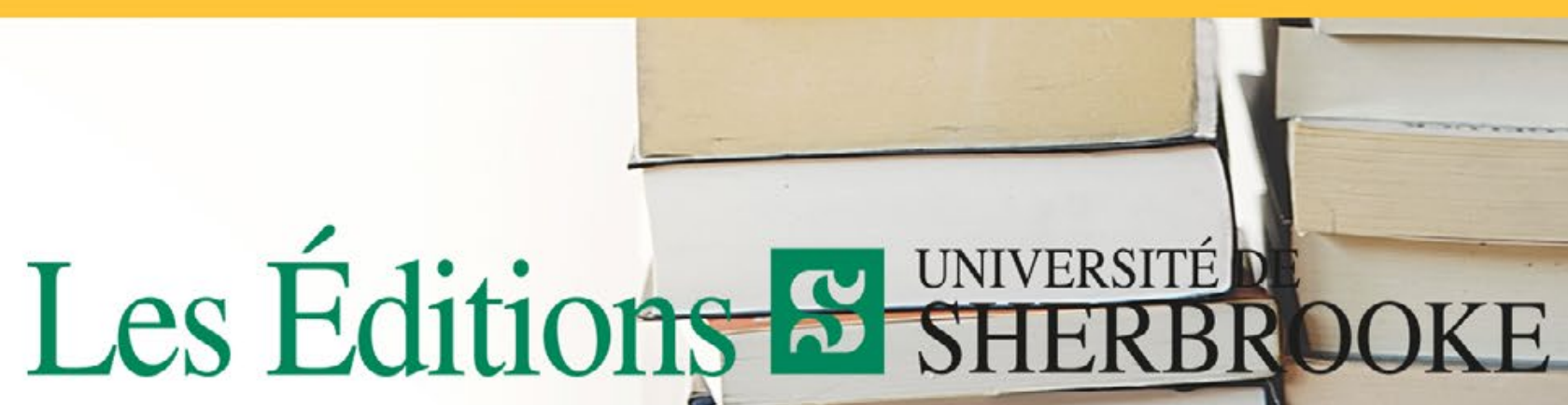




\section{Remerciements}

NOUS AIMERIONS REMERCIER CHALEUREUSEMENT LES AUTEURES POUR LEUR TRAVAIL DE QUALITÉ ET, SURTOUT, POUR LEUR PATIENCE PENDANT TOUT LE PROCESSUS D'ÉVALUATION PAR LES PAIRS. NOUS TENONS ÉGALEMENT À EXPRIMER TOUTE NOTRE RECONNAISSANCE AUX PERSONNES QUI ONT AGI À TITRE D'ARBITRE EN DONNANT DE LEUR TEMPS ET EN METTANT À PROFIT LEUR EXPERTISE À TRAVERS CET OUVRAGE.

LES ÉDITIONS DE L'UNIVERSITÉ DE SHERBROOKE, 2016.

ISBN: 978-2-7622-0354-7

URI: HTTP://HDL.HANDLE.NET/11143/8815

DOI: HTTP://DX.DOI.ORG/10.17118/11143/8815 


\section{Table des matières}

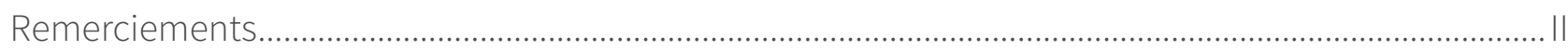

Introduction:

pour une plus grande reconnaissance de la recherche innovante en éducation...................................2

Assurer la rigueur scientifique de la recherche-action ..........................................................................

Enseigner la lecture au primaire :

un aperçu des pratiques d'enseignants des 2e et 3e cycles du primaire 22

Recherche-action-formation au préscolaire et au 1er cycle du primaire:

pistes méthodologiques.....

Intégration de la multimodalité dans les projets d'innovation de stagiaires en formation 69

Dispositif didactique pour développer la lecture littéraire au deuxième cycle du secondaire...... .98 
TITRE: INTRODUCTION : POUR UNE PLUS GRANDE RECONNAISSANCE DE LA RECHERCHE INNOVANTE EN ÉDUCATION.

Auteur(s): Isabelle Carignan, Ph.D., TÉlé-université (TÉlUQ), Marie-Christine Beaudry, Ph.D., Université du Québec à Montréal (UQAM), François Larose, Ph.D., Université de SherbroOke

PuBLICATION: LA RECHERCHE-ACTION ET LA RECHERCHE-DÉVELOPPEMENT AU SERVICE DE LA LITTÉRATIE.

PAGES: $1-5$

Directeurs: ISABelle Carignan, Marie-Christine Beaudry et François LaRose

ÉdiTEUR: LES ÉdITIONS DE L'UNIVERSITÉ DE SHERBROOKE, 2016.

ISBN: 978-2-7622-0354-7

URI: HTTP://HDL.HANDLE.NET/11143/8806

DOI: HTTP://DX.DOI.ORG/10.17118/11143/8806 


\title{
INTRODUCTION : pour une plus grande reconnaissance de la recherche innovante en éducation
}

\author{
Isabelle Carignan, Ph.D., Télé-université (TÉLUQ) \\ Marie-Christine Beaudry, Ph.D., Université du Québec à Montréal (UQAM) \\ François Larose, Ph.D., Université de Sherbrooke
}

Les recherches en didactique du français, menées par des chercheurs issus de domaines disciplinaires divers, donnent lieu à des approches quantitatives et qualitatives tout aussi variées. Si la recherche-action est bien documentée, autant en éducation en général qu'en didactique du français en particulier, la recherche-développement le semble beaucoup moins (Loiselle et Harvey, 2007). Pourtant, toutes deux sont des méthodologies employées pour notamment mener à terme des recherches visant à développer la littératie au primaire et au secondaire. Le premier volume de ce collectif présente cinq articles qui s'attardent à la recherche-développement et à la recherche-action : l'un porte sur les critères de scientificité de la recherche-action, un autre fait état d'une recherche-action, deux présentent des recherches-actions-formations et, enfin, un article porte sur une recherche-développement.

Si la notion de littératie est fréquemment employée dans le milieu franco-ontarien (MÉO, 2006), elle le demeure relativement peu au Québec et dans la francophonie (Hébert et Lépine, 2013). Ce constat a d'ailleurs conduit Hébert et Lépine à dresser l'état de cette notion dans les écrits scientifiques francophones qui en proposent une définition. Il ressort de leur recension des écrits que la littératie constitue une "notion multidimensionnelle, dont les contours restent encore flous » (Hébert et Lépine, 2013, p. 27). Elle ne serait plus considérée, selon Kirsch (2005), comme « [...] une capacité acquise à l'école primaire, mais plutôt comme un ensemble évolutif de compétences, de connaissances et de stratégies qu'une personne met en œuvre tout au long de sa vie dans divers contextes ainsi que dans ses relations avec ses pairs et avec sa collectivité » (p. 11). En d'autres mots, la notion de littératie « est [donc] en lien avec le développement, chez les individus, de l'habileté à lire, à écrire, à parler et à écouter. [...] [c] es pratiques [dites] « littératiées » peuvent être utilisées dans la vie courante, à la maison, au travail, à l'école ou dans la collectivité en fonction des objectifs visés par chacun, et ce, en interaction avec ses propres valeurs et sa propre culture. » (Beauregard, Carignan et Létourneau, 2011, p. 8). 
Selon Hébert et Lépine (2013), l'emploi de la notion de littératie revêtirait plusieurs intérêts. Parmi ceux-ci, mentionnons que la littératie se développe à travers une variété de textes, qu'ils soient sur supports papier ou numérique, et qu'elle se travaille au travers de tâches «les plus authentiques possible» (Hébert et Lépine, 2013, p. 38). La littératie se développe tout au long de la vie et permet de s'adapter aux différentes situations quotidiennes auxquelles sont confrontés les individus. C'est en ce sens que s'inscrivent les textes de ce collectif en s'intéressant notamment aux apprenants du primaire et du secondaire qui ont été amenés à développer différentes compétences en lien avec la littératie, et ce, sur des supports variés.

Le premier article de Louise Bourgeois de l'Université Laurentienne porte sur les critères de scientificité de la recherche-action. L'auteure fait part, dans un premier temps, des critères de rigueur méthodologique et des critères relationnels s'appliquant à toutes les recherches de type qualitatif. Dans un deuxième temps, elle fait ressortir les critères qui ont pour but de soutenir la crédibilité des résultats de la recherche-action. Un tableau synthèse à la toute fin permet d'avoir un regard global sur ces critères et de juger adéquatement la rigueur scientifique de la recherche-action.

Le deuxième article, de Pascale Thériault et Nadine Laurin de l'Université du Québec à Chicoutimi (UQAC), est la présentation d'une recherche-action qui a été réalisée au primaire. Celle-ci est née à la suite d'un besoin manifesté par des enseignants de réfléchir à l'amélioration de leurs pratiques pédagogiques en lecture. Les objectifs de cette recherche sont, d'une part, d'identifier les stratégies de lecture enseignées par des enseignants des $2^{\mathrm{e}}$ et $3^{\mathrm{e}}$ cycles du primaire et, d'autre part, d'expérimenter des pistes d'amélioration des pratiques pédagogiques de ces enseignants en regard de l'enseignement de stratégies de lecture tout en réfléchissant à leur mise en place.

Le troisième article de Lizanne Lafontaine de l'Université du Québec en Outaouais (UQO) est très novateur, car il fait part d'un type de recherche encore peu documenté : la recherche-action-formation. En effet, cette chercheure a souhaité définir ce qu'est la recherche-action-formation et en présenter le désign méthodologique. Celui-ci est notamment illustré par la présentation d'une recherche-action-formation ayant comme objectif général d'offrir des séminaires de formation et de recherche à des praticiens œuvrant dans le milieu scolaire afin qu'ils puissent créer et expérimenter des situations d'apprentissage et d'évaluation (SAÉ) intégrant la littératie.

Le quatrième article, écrit par Nathalie Lacelle et Amal Boultif de l’Université du Québec à Montréal (UQAM), a pour but de faire évoluer les pratiques d'enseignement en littératie médiatique mutimodale par l'entremise des stagiaires en formation initiale des maitres. Deux questions fondamentales sous-tendent cette recherche. Dans un premier temps, ces chercheures se questionnent à savoir comment la recherche-action-formation peut favoriser le transfert de connaissances issues de la recherche à la pratique de stagiaires en formation. Dans un deuxième temps, la question posée va comme suit: quelles sont les incidences de ce transfert sur le développement de nouvelles pratiques didactiques? Un article innovant sur la manipulation des savoirs des enseignants stagiaires à l'aide de la multimodalité et des technologies. 
Enfin, le cinquième article de Marie-Christine Beaudry de l'Université du Québec à Montréal (UQAM) est une recherche-développement. Dans la foulée des travaux sur la didactique de la lecture littéraire, elle s'est intéressée à l'établissement d'un rapport vivant entre le sujet lecteur et le texte littéraire. Cette recherche a pour buts 1 ) d'élaborer un dispositif didactique en trois volets (le questionnement réciproque, l'écrit de travail et l'écriture d'invention) susceptible de développer la lecture littéraire chez des élèves de la cinquième secondaire à partir d'un roman contemporain québécois; 2) d'évaluer ce dispositif didactique et; 3) d'améliorer le dispositif didactique créé. Grâce à cet article inédit, nous pouvons constater que l'élaboration d'outils didactiques pour soutenir adéquatement le développement de la lecture littéraire s'avère encore crucial pour le milieu scolaire.

Enfin, le but de ce collectif est de redonner ses lettres de noblesse à des types de recherche «mal aimés » ou « moins valorisés » que sont la recherche-action et la recherche-développement. Pourtant, ces types de recherche sont nécessaires, voire primordiaux, dans la formation pratique. En effet, il s'agit de recherches qui mettent à l'épreuve la théorie (Guichon, 2007; Poussard, 2000) et qui créent des liens entre les théories de l'enseignement, les théories de l'apprentissage ainsi que les pratiques réelles du milieu scolaire. 


\section{Références}

Beauregard, F., Carignan, I. et Létourneau, M.-D. (2011). Recension des écrits scientifiques sur la littératie familiale et communautaire (Rapport). Québec: Ministère de l'Éducation, du Loisir et du Sport (MELS). Repéré à https://depot.erudit.org/bitstream/003789dd/1/Beauregard_Carignan_MELS_litteratie_familiale.pdf

Gouvernement de l'Ontario (2006). Le curriculum de l'Ontario de la 1're à la 8e année. Français. Ottawa, Ontario : ministère de l'Éducation.

Hébert, M. et M. Lépine (2013). De l'intérêt de la notion de littératie en francophonie : un état des lieux en sciences de l'éducation. Globe. Revue internationale d'études québécoises, 16(1), 25-43.

Kirsch, I. (2005). Comprendre ce qui a été mesuré dans EIACA (2005). L’Enquête internationale sur l'alphabétisation des adultes (EIAA).

Loiselle, J. et Harvey, S. (2007). La recherche développement en éducation : fondements, apports et limites. Recherches qualitatives, 27(1), 40-59.

Poussard, C. (2000). La compréhension de l'anglais oral et les technologies éducatives. (Thèse de doctorat). Université Paris Diderot - Paris 7, Paris.

Cet ouvrage est conforme à la nouvelle orthographe.

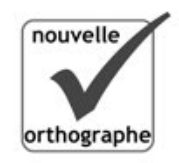


TITRE: ASSURER LA RIGUEUR SCIENTIFIQUE DE LA RECHERCHE-ACTION

Auteur(s): LOUISE BOURGEOIS, UNIVERSITÉ LAURENTIENNE

PublicATION: LA RECHERCHE-ACTION ET LA RECHERCHE-DÉVELOPPEMENT AU SERVICE DE LA LITTÉRATIE.

PAGES: $6-20$

DiRecteurs: ISABELle CARIgnAN, MARIE-Christine BeAudry ET FrançOIS LAROSE ÉdiTEUR: LES ÉdITIONS DE L'UNIVERSITÉ DE SHERBROOKE, 2016.

ISBN: 978-2-7622-0354-7

URI: HTTP://HDL.HANDLE.NET/11143/8807

DOI: HTTP://DX.DOI.ORG/10.17118/11143/8807 


\section{Assurer la rigueur scientifique de la recherche-action}

Louise Bourgeois, EAO, M.Éd., doctorante à l'Université d'Ottawa

Professeure à l'Université Laurentienne

Résumé : Les critères auxquels on peut se référer pour juger de la qualité et de la rigueur d'une recherche varient selon la posture épistémologique des chercheurs. Alors que la recherche positiviste s’appuie sur des critères de validité scientifique et que la recherche interprétative est soutenue par des critères méthodologiques et relationnels, qu'en est-il de modèles de recherche comme la recherche-action? Cet article vise d'abord à rappeler les critères de rigueur de la recherche interprétative, à faire ressortir les critères qui s'appliquent spécifiquement à la recherche-action et, enfin, à proposer des moyens pour satisfaire à chacun de ces critères.

Mots-clés : critères de scientificité, rigueur, recherche-action, moyens. 
La question de la valeur scientifique d'une recherche préoccupe tous les chercheurs, et ce, peu importe leur posture épistémologique. Alors que les critères de scientificité de la recherche positiviste sont bien ancrés dans la validité scientifique, ceux de la recherche interprétative relèvent davantage de la rigueur méthodologique et relationnelle (Anadón, 2006; Gohier, 2004; Lincoln, 1995). Mais qu'en est-il de modèles de recherche comme la recherche-action qui, bien qu'elle vise la production de savoir, entretient également des visées de changement dans la pratique professionnelle? Cet article vise deux objectifs : 1) il cherche à rappeler les critères de rigueur méthodologique et les critères relationnels qui s'appliquent à toutes les recherches qui se situent dans le giron qualitatif et 2) il a également pour but de faire ressortir les critères qui visent spécifiquement à soutenir la crédibilité des résultats de la recherche-action. Par ailleurs, pour chacun de ces objectifs, il est également question d'évoquer des exemples de mécanismes à mettre en place dans la recherche pour satisfaire a) les critères de rigueur méthodologiques (crédibilité, transférabilité, fiabilité et constance interne), b) les critères de rigueur relationnels (équilibre et authenticité) et c) les critères propres à la rechercheaction en fonction des modèles adoptés (respect des valeurs et des principes démocratiques, faisabilité, appropriation et cohérence systémique).

\section{Critères de rigueur méthodologique}

C'est dans le contexte du renouveau du paradigme interprétatif des années 1950 qu'ont commencé à émerger des critères de scientificité propres aux méthodes qualitatives (Laperrière, 1997). Le milieu du $20^{e}$ siècle a marqué une période pendant laquelle les tenants du paradigme interprétatif cherchaient à redéfinir les critères de validité du positivisme conventionnel dans le but de tenir compte des caractéristiques particulières de la recherche interprétative. En effet, le but positiviste de chercher à neutraliser la subjectivité dans la recherche n'était pas pertinent à la recherche interprétative justement parce que celle-ci adhère à une position selon laquelle la connaissance est subjective puisqu'elle est liée au vécu du sujet (Guba et Lincoln, 2005). Ainsi, Lincoln et Guba (Guba, 1981; Lincoln et Guba, 1985) ont élaboré des critères méthodologiques parallèles aux critères de validité du positivisme qui visent avant tout à tenir compte de la subjectivité de la recherche interprétative et des effets de l'interaction entre le chercheur et le sujet (Laperrière, 1997). Laperrière (1997) explique que:

[...] les chercheurs qualitatifs voulaient maximiser la validité de leurs résultats en balisant ou en exploitant les ressources de la subjectivité plutôt qu'en essayant de l'exclure des processus de recherche, puis en encourageant une étude des phénomènes sociaux en contexte naturel plutôt que dans un contexte qu'on tentait de neutraliser au départ pour en arriver à établir rapidement des généralisations. (p. 367)

Quatre critères liés aux méthodes et aux procédures d'analyse de la recherche interprétative ont été définis en parallèle à ceux du paradigme positiviste. Il s'agit des critères de crédibilité, de transférabilité, de fiabilité et de constance interne (Lincoln et Guba, 1985). 


\section{Crédibilité}

Parallèlement à la validité interne qui vise à vérifier la capacité de la recherche positiviste à produire des données attribuables à l'intervention plutôt qu'à d'autres phénomènes (Cohen, Manion et Morrison, 2011), le critère de crédibilité en recherche interprétative s'intéresse à vérifier la congruence entre le sens véhiculé par le sujet et le sens dégagé par le chercheur, notamment dans la collecte, l'analyse et l'interprétation des données (Gohier, 2004; Lincoln et Guba 1985; Savoie-Zajc, 2011). Au bout du compte, le critère de crédibilité vise à répondre à la question : sommes-nous devant un portrait authentique de ce qui a été observé? Alors que l'engagement prolongé du chercheur sur le terrain, la triangulation des méthodes et le journal réflexif du chercheur sont toutes des techniques qui permettent de soutenir la crédibilité des résultats de la recherche, la vérification du texte de la recherche par les sujets est considérée par Lincoln et Guba (1985) comme la plus importante stratégie pour établir la crédibilité.

\section{Transférabilité}

Une importante visée du paradigme positiviste est de développer et de vérifier des règles généralisables à un grand nombre de contextes différents (Cohen, Manion et Morrison, 2011). Il s'agit de la validité externe. En recherche interprétative toutefois, l'échantillon d'étude n'est généralement pas statistiquement représentatif et, par conséquent, les résultats ne mènent pas à des généralisations (Guba, 1981). Ainsi, le critère de transférabilité vise à déterminer si les conclusions d'une recherche peuvent avoir un sens dans un autre contexte que celui étudié (Gohier, 2004; Pourtois et Desmet, 2007). Ce critère se partage entre le chercheur et celui qui cherche à utiliser les résultats de la recherche dans son milieu. II revient alors au chercheur de fournir des descriptions riches du contexte et de l'échantillon de l'étude et à l'utilisateur de s'interroger sur la pertinence et la ressemblance entre le contexte de la recherche et son propre milieu (Savoie-Zajc, 2011).

\section{Fiabilité}

L'objectivité en recherche quantitative, qui est liée à la neutralité des données observables et mesurables, revient en recherche interprétative à la fiabilité qui vise à démontrer la transparence du chercheur et, par le fait même, l'indépendance de ses interprétations par rapport à ses biais (Gohier, 2004; Pourtois et Desmet, 2007). À la base de ce critère « se trouve la lucidité du chercheur à l'égard de ses jugements et la reconnaissance de ceux-ci en tant qu'éléments influençant ses analyses et interprétations (triangulation interne du chercheur) (Mucchielli, 2009, p. 60).

Pour assurer la fiabilité de la recherche, la triangulation s'avère encore une fois la stratégie la plus utile, notamment la triangulation des observateurs ainsi que la triangulation interne du chercheur (Pourtois et Desmet, 2007). Le chercheur doit démontrer qu'il est conscient de sa subjectivité (Miles et Huberman, 2003) et prendre des mesures pour attester que les résultats émergent des données 
et non de ses intérêts ou de ses motivations. La collaboration de plusieurs chercheurs dans la recherche qui peuvent comparer leurs observations et leurs interprétations des données peut soutenir la fiabilité de la recherche. La vérification interjuges, par exemple, peut être utilisée pour assurer la fiabilité des résultats où les divergences entre les codeurs sont analysées et résolues par l'intermédiaire d'une analyse conjointe du matériel. À cet effet, Miles et Huberman (2003) recommandent un indice de fidélité d'au moins 70 \% avant les ajustements et de 90 \% après. Bien qu'il n'y ait pas d'indicateurs dans la littérature quant au nombre de codeurs à utiliser dans le processus, Kolbe et Burnette (1991) ont remarqué, dans une recension de recherches, qu'il s'agit le plus souvent de deux codeurs. De la même façon, il n'y a pas d'indicateurs dans la littérature quant au pourcentage du matériel qui devrait être recodé par différents codeurs pour assurer la fiabilité. Quand cette donnée est fournie dans les recherches interprétatives, on peut observer qu'elle peut varier entre $10 \%$ jusqu'à la totalité du corpus du matériel qui est recodé par au moins deux codeurs. Dans la littérature sur la fiabilité du codage, la formation et l'indépendance des juges semblent être une plus grande préoccupation.

\section{Constance interne}

Le critère de fidélité en recherche quantitative vise la stabilité et la reproductibilité des résultats (Cohen, Manion et Morrison, 2011); il a peu de sens dans un cadre qualitatif où le changement est fondamental. Si la fidélité, en recherche quantitative, vise la persistance d'une procédure de mesure à procurer la même réponse, la constance interne, en recherche interprétative, revient plutôt à "l'indépendance des observations et des interprétations par rapport à des variations accidentelles ou systématiques » (Gohier, 2004, p.7). En effet, la constance interne cherche à fournir un degré d'assurance que les résultats de la recherche n'ont pas été influencés, par exemple, par la personnalité du chercheur, les instruments utilisés ou les conditions de collecte de données (Mucchielli, 2009). Pour augmenter la constance interne de la recherche, Pourtois et Desmet (2007) citent de nouveau la triangulation, plus précisément la triangulation des chercheurs, où au moins deux chercheurs collaborent à toutes les étapes de la recherche, ainsi que la triangulation temporelle, où le chercheur tient compte de l'évolution du phénomène dans le temps. À défaut d'autres chercheurs participant à la recherche, la vérification par un chercheur externe peut servir aux fins de la triangulation des chercheurs (Creswell, 2007; Miles et Huberman, 2003).

Enfin, ces quatre critères (crédibilité, transférabilité, fiabilité et constance interne) visent à soutenir la rigueur méthodologique de la recherche interprétative, mais ils ne tiennent pas compte du caractère « dynamique, collaboratif et socioconstructiviste de l'approche interprétative » (Savoie-Zajc, 2000, p.171). Pour cette raison, des critères relationnels s'ajoutent aux critères méthodologiques dans le but de soutenir la qualité globale de la recherche puisqu'ils font davantage référence aux rapports qu'entretient le chercheur avec les sujets. 


\section{Critères relationnels}

Les critères relationnels élaborés par Guba et Lincoln (1989) n’ont pas d'équivalents en recherche quantitative puisqu'ils misent sur les relations qui s'établissent entre le chercheur et le sujet. Il s'agit du critère d'équilibre et des critères d'authenticité ontologique, éducative, catalytique et tactique. Manning (1997) souligne l'importance des critères relationnels pour soutenir la valeur de la recherche interprétative :

If the research fails to meet these commitments [balance of perspectives, learning by the researcher and respondents, shared knowledge and social action], the quality of the research (e.g. meaningfulness, usefulness, ability to enact social change) is questionable. (p. 94)

\section{Équilibre}

L'équilibre consiste à s'assurer que les points de vue de l'ensemble des sujets concernés par la recherche sont exprimés de façon équitable (Denzin et Lincoln, 2000; Gohier, 2004; Savoie-Zajc, 2011). Cet équilibre sous-entend que le chercheur doit fournir l'occasion, voire convaincre les sujets, d'exprimer leurs idées et en tenir compte, qu'il soit en accord ou en désaccord avec celles-ci. Savoie-Zajc (2011) mentionne que l'équilibre exige une certaine pondération des divers points de vue dans le texte. En effet, le critère d'équilibre cherche à amener le chercheur à se poser deux questions fondamentales:

1. Est-ce que tous les sujets ont eu l'occasion d'exprimer leurs idées pendant la recherche?

2. Est-ce que les points de vue de l'ensemble des sujets sont exprimés de façon équitable dans la recherche?

Pour qu'il y ait équilibre, Guba et Lincoln (1989) suggèrent des stratégies telles que la vérification du texte de la recherche par les sujets, l'implication de plus d'un chercheur à toutes les étapes de la recherche et l'engagement prolongé des chercheurs sur le terrain dans le but d'établir des rapports positifs avec les sujets.

\section{Authenticité}

L'authenticité se répartit en quatre critères : l'authenticité ontologique, l'authenticité éducative, l'authenticité catalytique et l'authenticité tactique.

L'authenticité ontologique vise à démontrer que les sujets ont amélioré leurs connaissances et élargi leurs représentations à propos de l'objet ou du phénomène à l'étude (Gohier, 2004; Savoie-Zajc, 2011). Cette transformation amène les participants à se construire des représentations plus riches et complexes de la réalité (Gohier, 2004). On peut penser, par exemple, à une entrevue où les sujets sont 
confrontés à des questions auxquelles ils n’ont jamais pensé et sont obligés de formuler de nouvelles idées pour y répondre.

L'authenticité éducative vise l'approfondissement de la connaissance de soi et une meilleure compréhension des représentations des autres participants (Gohier, 2004; Savoie-Zajc, 2011). La recherche oriente premièrement le sujet vers une prise de conscience de sa propre position pour ensuite mener à la comparer à celles des autres. L'intention est de faire en sorte que tous les participants acquièrent une compréhension plus riche, plus profonde et plus nuancée de leurs représentations et de celles des autres.

L'authenticité catalytique mise sur les apprentissages suscités par la recherche et vise des retombées au niveau du désir et du pouvoir d'action des sujets (Gohier, 2004; Savoie-Zajc, 2011). La recherche, par sa pertinence, devient ainsi un catalyseur qui incite à l'action. Ce critère encadre la recherche qui aborde un problème authentique, fait en sorte que sa finalité ne se limite pas qu'à la production de savoirs et met en place les conditions pour que l'engagement des sujets et du chercheur dépasse le contexte de la recherche.

L'authenticité tactique fournit les outils conceptuels aux participants qui leur permettent de passer à l'action (Gohier, 2004; Savoie-Zajc, 2011). Selon ce critère, le chercheur reconnait que les sujets qui participent à une recherche n'ont pas tous les mêmes habiletés et compétences. II s'engage ainsi à les former, selon leurs besoins et leurs désirs, à des formes spécifiques d'action politique ou sociale. Il s'agit d'une façon de tenter de rétablir un équilibre social dans le but de donner aux sujets les moyens et le soutien pour être en mesure de produire un changement.

Ces critères d'authenticité se vérifient généralement grâce aux témoignages des sujets au cours et au terme de la recherche. Le chercheur peut, par exemple, avoir recours aux témoignages des sujets attestant de l'élargissement de leurs connaissances, de l'amélioration de leurs pratiques ou de l'acquisition d'outils les habilitant à passer à l'action, mais il doit également être sensible et attentif à la qualité des liens qu'il établit progressivement avec le sujet. En effet, le chercheur qui situe sa posture dans le paradigme interprétatif doit être en mesure de s'occuper des incidents qui peuvent nuire à l'authenticité des rapports avec les sujets et, par le fait même, réduire les bienfaits de leur participation à la recherche.

\section{Critères de rigueur de la recherche-action selon les modèles adoptés}

Van der Maren (1996) affirme que toute recherche interprétative doit d'abord respecter les critères de base de la recherche scientifique, notamment les critères méthodologiques et relationnels déjà mentionnés. II ajoute toutefois que chaque modèle de recherche a ses particularités et doit donc retenir les critères de rigueur qui lui sont propres. 
Par exemple, on admet que les critères de validité d'une recherche descriptive ne sont pas les mêmes que ceux d'une analyse interprétative ou d'une théorie de l'action : elles n'ont pas les mêmes fonctions, elles n'ont pas les mêmes prétentions et elles ne doivent pas ni ne peuvent pas être évaluées selon des critères identiques. (p. 1)

La recherche-action s'insère généralement dans un paradigme pragmatico-interprétatif (Savoie-Zajc, 2001) : interprétatif, car l'objectif est de comprendre le sens que les acteurs donnent à leur réalité, et pragmatique, car le savoir qui ressort de la recherche a des implications pratiques, utiles et concrètes dans leur vie professionnelle (Debuyist, Digneffe et Pires, 2008; Morgan, 2007). Elle se distingue des autres types de recherche interprétative selon trois composantes fondamentales, soit l'intention, les rôles et la démarche méthodologique. L'intention de la recherche-action est de produire un changement dans un milieu professionnel donné et d'améliorer les pratiques (Dolbec et Clément, 2000; Savoie-Zajc, 2001). Par ailleurs, sur le plan des rôles, la recherche-action implique les acteurs à titre de cochercheurs. En effet, Gauthier (1984) indique qu'il s'agit d'une « modalité de recherche qui rend l'acteur chercheur et qui fait du chercheur un acteur. » (p. 522). Ce rapport symétrique entre chercheurs et praticiens à toutes les étapes de la recherche fait en sorte que la production du savoir est validée dans l'action et dans la pratique. Enfin, pour que l'amélioration de la pratique soit possible, la recherche-action emprunte une démarche méthodologique de résolution de problèmes selon un cycle de planification, d'action, d'observation et de réflexion (Dolbec et Clément, 2000). En effet, ces trois composantes font en sorte que les critères de rigueur méthodo logique et les critères relationnels de la recherche interprétative ne suffisent pas pour assurer la rigueur de la recherche-action. C'est la raison pour laquelle Savoie-Zajc (2001) suggère quatre nouveaux critères qui tiennent compte des particularités de la recherche-action, soit les critères du respect des valeurs et des principes démocratiques, de faisabilité, d’appropriation ainsi que de cohérence systémique.

\section{Respect des valeurs et des principes démocratiques}

Le critère du respect des valeurs et des principes démocratiques s'associe au groupe de critères relationnels qui mettent de l'avant l'éthique des procédures (Gohier, 2004). Ce critère renvoie effectivement à l'attitude du chercheur par rapport aux sujets (cochercheurs) et à sa responsabilité de garantir la qualité des échanges, par exemple, en mettant en place un climat de travail propice à l'interaction et à la collaboration, en protégeant les droits des participants à s'exprimer librement et en s'assurant d'inclure tous les participants dans la prise de décision à toutes les étapes de la recherche (Dolbec et Clément, 2000; Savoie-Zajc, 2001). Pour vérifier et témoigner du respect des valeurs et des principes démocratiques dans la recherche, Savoie-Zajc (2001) suggère la tenue d'un journal de bord par tous les membres qui participent à la recherche ainsi que des rencontres périodiques d'objectivation afin de discuter ouvertement de situations qui émergent et d'apporter, au besoin, des ajustements au processus de recherche. 


\section{Faisabilité}

Toujours dans l'ordre de l'éthique des procédures, le critère de faisabilité renvoie à la pertinence de la recherche-action pour le groupe, à la planification d'un projet qui tient compte des contraintes du milieu et à la viabilité du changement ou des solutions que les résultats proposent (Savoie-Zajc, 2001). Dans ce contexte de résolution de problèmes, les solutions mises de l'avant doivent orienter les actions et les décisions des participants tout en tenant compte de la réalité du milieu. À quoi bon mettre sur pied un projet dont la question est peu pertinente pour le milieu? Pour quelle raison s'acharner à discuter de solutions qui ne pourront effectivement pas être mises en œuvre? Le critère de faisabilité exige que le projet soit centré sur un problème réel de la profession et que les solutions répondent aux particularités du milieu (p. ex. : économiques, culturelles, politiques). Pour assurer la faisabilité du projet, Savoie-Zajc (2001) recommande d'établir des liens étroits avec le milieu (p. ex. : dirigeants, administration) et de maintenir une communication ouverte avec celui-ci pour pouvoir obtenir l'information nécessaire permettant de prendre des décisions éclairées au regard de la faisabilité du projet et de la mise en œuvre des solutions. En maintenant une communication continue avec les partenaires au regard du projet, la pertinence de la recherche et la viabilité des résultats dans le milieu sont soutenues.

\section{Appropriation}

Le critère d'appropriation rappelle l'importance d'assurer l'engagement des sujets à poursuivre la démarche initiée par la recherche (Dolbec et Clément, 2000; Savoie-Zajc, 2001). Il s'aligne avec les critères d'authenticité déjà abordés qui visent l'élargissement des connaissances des sujets, l'amélioration de leurs pratiques et l'acquisition d'outils les habilitant à passer à l'action. Ces éléments font partie intégrante de la recherche-action et représentent un enjeu incontournable qui consiste à amener les participants à s'approprier les résultats et à leur proposer des occasions de poursuivre la réflexion entamée dans le cadre de la recherche. La finalité de la recherche-action ne s'arrête pas à la production de savoirs, mais s'étend aussi à produire un changement durable et à transformer la pratique professionnelle des sujets. Le critère d'appropriation traite de la qualité des changements, c'est-à-dire de l'apprentissage et des prises de conscience des individus concernés par la recherche et de l'incidence de la participation sur la pratique professionnelle, et ce, à long terme. Bien que le journal de bord tenu par les différents membres du groupe de recherche-action et des rencontres périodiques d'objectivation puissent soutenir la pertinence et la faisabilité des solutions dans le milieu, seul un suivi longitudinal de la part du chercheur principal ou encore d'un vérificateur externe pourra permettre de déterminer jusqu'à quel point les cochercheurs se sont approprié les résultats de la recherche dans le but d'améliorer la pratique professionnelle de façon durable. 


\section{Cohérence systémique}

Le critère de cohérence systémique s'ajoute aux critères méthodologiques et réfère à la cohérence de la démarche de recherche et à la rigueur de la collecte et de l'analyse des données (Savoie-Zajc, 2001). Il se rapproche du critère de crédibilité et de fiabilité qui demande des descriptions riches, claires et détaillées du processus de recherche, l'accès aux données brutes et la documentation des influences diverses qui ont pu avoir une influence sur les résultats. Le critère de cohérence systémique est le point d'ancrage de la recherche-action puisqu'il assure l'alignement de la méthodologie sur les objectifs visés, la collecte et l'analyse de données ainsi que les résultats. Il souligne également l'importance de documenter les rôles et les responsabilités des cochercheurs et garantit un processus rigoureux de collecte et d'analyse de données. Pour s'assurer de la cohérence systémique de la recherche, la triangulation s'avère encore une fois la stratégie la plus utile, notamment la triangulation des observateurs, où des pairs vérifient l'alignement de la méthodologie.

La recherche-action est une démarche complexe qui implique non seulement l'interaction avec des sujets agissant sur un terrain généralement difficile à comprendre et dont les propos ne sont pas faciles à traduire, mais également l'engagement au changement et à l'action dans des milieux qui peuvent parfois y résister. Dans ce contexte, la conception et la mise en œuvre d'une démarche méthodologie impliquant les cochercheurs et garante de validité est également complexe. Les critères de rigueur méthodologique et relationnelle dont il a été question dans cet article permettent de constituer une grille de lecture de la validité de la recherche-action. Le tableau 1 résume les divers critères de rigueur tout en éliminant certaines redondances et suggère également des moyens à mettre en place pour soutenir des données et des résultats crédibles. Bien que le nombre de critères s'élève à onze, ils peuvent être regroupés selon trois composantes de la recherche-action : le savoir, le pouvoir et le vouloir (Pourtois, Desmet et Humbeeck, 2013) :

- la production d’un savoir qui est valide - les critères de crédibilité, de transférabilité, de fiabilité et de constance interne;

- le partage du pouvoir dans la recherche qui doit se faire dans le respect de l'autre et l'implication de tous - les critères d'équilibre et du respect des valeurs et des principes démocratiques;

- le vouloir agir pour instaurer l'amélioration durable de la pratique professionnelle - les critères d'authenticité ontologique, d'authenticité éducative, de faisabilité et d'appropriation qui englobent l'authenticité catalytique et tactique.

Au regard du nombre de critères de rigueur de la recherche-action et du nombre de mécanismes pour en témoigner, deux questions se posent:

1. À combien de critères de rigueur une recherche doit-elle obligatoirement satisfaire?

2. Combien de mécanismes doit-on intégrer à la recherche pour satisfaire à un critère de rigueur? 
Devant ces deux préoccupations, le point le plus important est la rigueur méthodologique et relationnelle à toutes les étapes de la recherche, de la méthode à la présentation des résultats. On peut difficilement penser, par exemple, que la faisabilité d'une recherche-action est plus importante que la crédibilité. En effet, il faut documenter suffisamment toutes les phases de la recherche pour satisfaire à la plupart, sinon à tous les critères de rigueur méthodologique et relationnelle. Bien qu'il existe des protocoles bien établis en recherche, il n'en demeure pas moins que le jugement du chercheur est primordial en recherche interprétative et que la charge de la preuve de rigueur lui revient ultimement. 


\section{Tableau 1 : Critères et moyens pour juger la rigueur de la recherche-action}

\begin{tabular}{|c|c|c|}
\hline Critères & Questionnement possible & Exemples de mécanismes pour satisfaire au critère \\
\hline Crédibilité & $\begin{array}{l}\text { - Les sujets se retrouvent-ils dans le texte de la } \\
\text { recherche? } \\
\text { - Est-ce que la méthodologie est décrite de façon } \\
\text { assez détaillée? } \\
\text { - Est-ce que l'analyse des données est le résultat } \\
\text { d'une démarche rigoureuse? }\end{array}$ & $\begin{array}{l}\text { - Retour aux sujets pour valider le texte de la recherche; } \\
\text { - Engagement prolongé dans le milieu pour s'approprier la culture du milieu et } \\
\text { - Demprendre le contexte; } \\
\text { méthodes. }\end{array}$ \\
\hline Transférabilité & $\begin{array}{l}\text { - Est-ce que le milieu est décrit de façon suffisante } \\
\text { pour que celui qui cherche à utiliser les résultats de } \\
\text { la recherche puisse faire des liens avec son propre } \\
\text { milieu? } \\
\text { - Est-ce que les sujets qui participent à la recherche } \\
\text { sont clairement décrits? }\end{array}$ & $\begin{array}{l}\text { - Description riche et détaillée de l'école et de son contexte; } \\
\text { - Description détaillée des sujets de la recherche. }\end{array}$ \\
\hline Fiabilité & $\begin{array}{l}\text { - Le chercheur est-il conscient de ses biais? } \\
\text { - La recherche est-elle exempte de préjugés? }\end{array}$ & $\begin{array}{l}\text { - Journal de bord du chercheur pour mettre en évidence la } \\
\text { réflexion sur les biais, les présupposés; } \\
\text { - Implication de plus d'un chercheur à toutes les étapes de la } \\
\text { recherche (p. ex. : vérification intercodeurs); } \\
\text { - Triangulation des sources de données (p. ex. : entretien semi- } \\
\text { dirigé, observation, analyse de documents) pour rendre compte d'un même } \\
\text { phénomène. }\end{array}$ \\
\hline Constance interne & $\begin{array}{l}\text { - Les données sont-elles accessibles (p. ex., enregis- } \\
\text { trements, transcription d'entretiens)? } \\
\text { - Les données ont-elles été recueillies avec des } \\
\text { procédures rigoureuses? }\end{array}$ & $\begin{array}{l}\text { - Description riche et détaillée des procédures de collecte de données; } \\
\text { - Journal de bord pour documenter l'évolution de la recherche et les justifications } \\
\text { des décisions (p. ex. : raffinement des questions de recherche); } \\
\text { - Triangulation des chercheurs à toutes les étapes de la recherche (p. ex. : collecte } \\
\text { de données). }\end{array}$ \\
\hline $\begin{array}{l}\text { Cohérence } \\
\text { systémique }\end{array}$ & $\begin{array}{l}\text { - Est-ce que la démarche de la recherche est } \\
\text { cohérente (p. ex. : alignement entre la méthodolo- } \\
\text { gie, les objectifs visés, la collecte et l'analyse des } \\
\text { données)? } \\
\text { - Est-ce que les rôles et responsabilités des membres } \\
\text { sont documentés? }\end{array}$ & $\begin{array}{l}\text { - Vérification externe dans le but de porter un jugement sur la } \\
\text { démarche de la recherche; } \\
\text { - Justification des instruments de collecte de données; } \\
\text { - Documentation des rencontres (p. ex. : agenda, procès-verbaux ou comptes } \\
\text { rendus); } \\
\text { - Description riche et détaillée des procédures de collecte et d'analyse de don- } \\
\text { nées. }\end{array}$ \\
\hline
\end{tabular}




\begin{tabular}{|c|c|c|}
\hline Critères & Questionnement possible & Exemples de mécanismes pour satisfaire au critère \\
\hline Équilibre & $\begin{array}{l}\text { - Est-ce que tous les sujets ont eu l'occasion d'expri- } \\
\text { mer leurs idées pendant la recherche? } \\
\text { - Est-ce que les points de vue de l'ensemble des } \\
\text { sujets sont exprimés de façon équitable dans la } \\
\text { recherche? }\end{array}$ & $\begin{array}{l}\text { - Retour aux sujets pour valider le texte de la recherche; } \\
\text { - Engagement prolongé dans le milieu pour s'approprier la culture du milieu et } \\
\text { comprendre le contexte; } \\
\text { - Implication de plus d'un chercheur à toutes les étapes de la } \\
\text { recherche. }\end{array}$ \\
\hline $\begin{array}{l}\text { Authenticité } \\
\text { ontologique }\end{array}$ & $\begin{array}{l}\text { - Les sujets ont-ils amélioré leurs connaissances et } \\
\text { élargi leurs représentations à propos de l'objet ou } \\
\text { du phénomène à l'étude? } \\
\text { - Les sujets ont-ils des représentations plus riches } \\
\text { et plus complexes de l'objet ou du phénomène à } \\
\text { l'étude? }\end{array}$ & $\begin{array}{l}\text { - Témoignages des participants attestant de l'élargissement } \\
\text { de leurs connaissances et de l'amélioration de leur pratique } \\
\text { professionnelle. }\end{array}$ \\
\hline $\begin{array}{l}\text { Authenticité } \\
\text { éducative }\end{array}$ & $\begin{array}{l}\text { - Les sujets ont-ils approfondi leur connaissance } \\
\text { d'eux-mêmes? } \\
\text { - Les sujets ont-ils une meilleure compréhension des } \\
\text { représentations que se font les autres enseignants? }\end{array}$ & - Témoignages des participants attestant de l'élargissement de leurs perspectives. \\
\hline $\begin{array}{l}\text { Respect des valeurs } \\
\text { et des principes } \\
\text { démocratiques }\end{array}$ & $\begin{array}{l}\text { - Est-ce que les droits des membres du groupe sont } \\
\text { respectés? } \\
\text { - Le climat des rencontres est-il propice aux } \\
\text { échanges? } \\
\text { - Est-ce que tous les membres du groupe sont impli- } \\
\text { qués dans la prise de décisions de la recherche? }\end{array}$ & $\begin{array}{l}\text { - Journal de bord des cochercheurs; } \\
\text { - Rencontres d'objectivation; } \\
\text { - Vérification externe. }\end{array}$ \\
\hline Faisabilité & $\begin{array}{l}\text { Est-ce que la recherche et les solutions tiennent } \\
\text { compte des contraintes du milieu scolaire (p. ex., } \\
\text { contraintes sociales, politiques, économiques, } \\
\text { culturelles)? }\end{array}$ & $\begin{array}{l}\text { - Établissement de lien avec le milieu (p. ex. : partenaires, } \\
\text { dirigeants) pour vérifier la pertinence du projet et la viabilité des solutions dans } \\
\text { le milieu. }\end{array}$ \\
\hline $\begin{array}{l}\text { Appropriation } \\
\text { Authenticité } \\
\text { catalytique } \\
\text { Authenticité tactique }\end{array}$ & $\begin{array}{l}\text { - Est-ce que tous les membres se sont pleinement } \\
\text { engagés dans la recherche? } \\
\text { - Les membres ont-ils les outils nécessaires pour } \\
\text { passer à l'action? } \\
\text { - Les sujets ont-ils poursuivi la réflexion après la } \\
\text { recherche? }\end{array}$ & $\begin{array}{l}\text { - Diffusion et présentation du rapport de recherche par les cochercheurs; } \\
\text { - Suivi longitudinal de la part du chercheur principal auprès des membres du } \\
\text { groupe; } \\
\text { - Vérification externe. }\end{array}$ \\
\hline
\end{tabular}




\section{Références}

Anadón, M. (2006). La recherche « qualitative ». De la dynamique de son évolution aux acquis indéniables et aux questionnements présents. Recherches qualitatives, 26(1), 5-31.

Cohen, L., Manion L. et Morrison, K. (2011). Validity and reliability. Dans L. Cohen, L. Manion et K. Morrison (dir.), Research Methods in Education (p. 179-216). London, New York: Routledge.

Creswell, J. W. (2007). Qualitative inquiry and research design: Choosing among five traditions. Thousand Oaks, CA: Sage Publications.

Denzin, N. K. et Lincoln, Y. S. (2005). Introduction: The discipline and practice of qualitative research. Dans N. K. Denzin et Y. S. Lincoln (dir.), The SAGE handbook of qualitative research (3e éd., p. 1-32). Thousand Oaks, CA : Sage.

Dolbec, A. et Clément, J. (2000). La recherche-action. Dans L. Savoie-Zajc (dir.), Introduction à la recherche en éducation (p. 199-224). Sherbrooke : Éditions du CRP.

Gauthier, B. (1984). Recherche sociale. Québec: Presses de l’Université du Québec.

Gohier, C. (2004). De la démarcation entre critères d'ordre scientifique et d'ordre éthique en recherche interprétative. Recherches qualitatives, 24, 3-17.

Guba, E. G. (1981). Criteria for assessing the trustworthiness of naturalistic inquiries. Educational Communication and Technology Journal, 29(2), 75-91.

Guba, E. G. et Lincoln, Y. S. (1989). Judging the quality of fourth generation evaluation. Dans E. G. Guba et Y. S. Lincoln (dir.), Fourth Generation Evaluation (p. 229-251). London : Sage.

Guba, E. G. et Lincoln, Y. S. (2005). Paradigmatic controversies and emerging confluences. Dans N. K. Denzin, et Y. S. Lincoln (dir.), The SAGE handbook of qualitative research (3 éd., p. 191-216). Thousand Oaks, CA : Sage.

Kolbe, R. H. et Burnett, M. S. (1991). Content-analysis research: An examination of applications with directives for improving research reliability and objectivity. Journal of Consumer Research, 18, 243-250.

Laperrière, A. (1997). Les critères de scientificité des méthodes qualitatives. Dans J. Poupart, J.-P. Deslauriers, L.-H. Groulx, A. Laperrière, R. Mayer et A. Pires (dir.), La recherche qualitative. Enjeux épistémologiques et méthodologiques (p. 365-388). Montréal : Gaëtan Morin.

Lincoln, Y. S. (1995). Emerging criteria for quality in qualitative and interpretive research. Qualitative Inquiry, 1(3), 275-289.

Lincoln, Y. S. et Guba, E. G. (1985). Naturalistic inquiry. Beverly Hills CA: Sage.

Manning, K. (1997). Authenticity in constructivist inquiry : Methodological considerations without prescriptions. Qualitative Inquiry, 3(1), 93-115. 
Miles, M. B. et Huberman, M. A. (2003). Analyse des données qualitatives (2éd.). Paris : De Boeck.

Mucchielli, A. (2009). Dictionnaire des méthodes qualitatives en sciences humaines ( $3^{e}$ éd.). Paris: Armand Colin.

Pourtois, J.-P. et Desmet, H. (2007). Épistémologie et instrumentation en sciences humaines (3e éd.). Wavre, Belgique : Mardaga.

Pourtois, J.-P., Desmet, H. et Humbeeck, B. (2013). La recherche-action, un instrument de compréhension et de changement du monde. Recherches qualitatives - Hors Série, 5, 99-111.

Savoie-Zajc, L. (2000). La recherche qualitative/interprétative en éducation. Dans T. Karsenti et L. Savoie-Zajc (dir.), Introduction à la recherche en éducation (p. 171-198). Sherbrooke : Éditions du CRP.

Savoie-Zajc, L. (2001). La recherche-action en éducation : ses cadres épistémologiques, sa pertinence, ses limites. Dans M. Anadón et M. L'Hostie (dir.), Nouvelles dynamiques de recherche en éducation (p. 15-49). Sainte-Foy: Les Presses de l'Université Laval.

Savoie-Zajc, L. (2011). La recherche qualitative/interprétative en éducation. Dans T. Karsenti et L. Savoie-Zajc (dir.), La recherche en éducation (p. 123-146). Saint-Laurent : Éditions RPI.

Van der Maren, J.-M. (1996, octobre). La recherche qualitative peut-elle être rigoureuse? Communication présentée au Colloque international Recherche qualitative et gestion, HEC, Montréal, Canada. 
TITRE: ENSEIGNER LA LECTURE AU PRIMAIRE : UN APERÇU DES PRATIQUES D'ENSEIGNANTS DES $2^{\mathrm{E}}$ ET $3^{\mathrm{E}}$ CYCLES DU PRIMAIRE

Auteur(s): Pascale Thériault, Ph.D., Professeure et Nadine Laurin, M.A., Doctorante, UNIVERSITÉ DU QUÉBEC À CHICOUTIMI

PuBlicATION: LA RECHERCHE-ACTION ET LA RECHERCHE-DÉVELOPPEMENT AU SERVICE DE LA LITTÉRATIE.

PAGES: $21-40$

Directeurs: ISABElle Carignan, Marie-Christine Beaudry ET FrançoIS LaROSE

ÉdITEUR: LES ÉDITIONS DE L'UNIVERSITÉ DE SHERBROOKE, 2016.

ISBN: 978-2-7622-0354-7

URI: HTTP://HDL.HANDLE.NET/11143/8808

DOI: HTTP://DX.DOI.ORG/10.17118/11143/8808 


\title{
Enseigner la lecture au primaire : un aperçu des pratiques d'enseignants des $2^{\mathrm{e}}$ et $3^{\mathrm{e}}$ cycles du primaire
}

\author{
Pascale Thériault, Ph.D., professeure \\ Nadine Laurin, M.A., doctorante \\ Université du Québec à Chicoutimi
}

Résumé : Apprendre à lire exige de développer un ensemble de stratégies et, dans un contexte d'enseignement-apprentissage, il s'avère nécessaire que les stratégies de lecture soient enseignées (Fisher, Frey et Lapp, 2008). Toutefois, il semble que les enseignants se sentent peu à l'aise avec l'enseignement des stratégies de lecture par la modélisation (Martel et Lévesque, 2010; Pressley et Afflerbach, 1995). Au Saguenay-Lac-Saint-Jean, des enseignants des $2^{\mathrm{e}}$ et $3^{\mathrm{e}}$ cycles du primaire ont exprimé le besoin de réfléchir sur leurs pratiques d'enseignement des stratégies de lecture afin d'apporter les ajustements nécessaires à un apprentissage de la lecture plus accompli chez leurs élèves. Pour y parvenir, douze enseignantes, un enseignant, deux conseillères pédagogiques et une chercheure se sont engagés dans une recherche-action (Dolbec et Clément, 2004) visant l'amélioration des pratiques relatives à l'enseignement de la lecture. Comme le suggère Couture (2011), le portrait des pratiques déjà mises en place par les enseignants a été dressé dans un premier temps afin d'ancrer le processus de changement à même le travail et les besoins des enseignants. Les résultats indiquent que ce sont les stratégies utiles avant la lecture qui seraient les plus enseignées, que cet enseignement s'effectuerait principalement à l'aide du matériel didactique, et ce, avec l'ensemble du groupe classe.

Mots-clés : stratégies de lecture, pratiques d'enseignement, modélisation, primaire, rechercheaction. 


\section{Introduction}

Les lecteurs compétents sont actifs tout au long de la lecture. Ils mobilisent plusieurs stratégies dans le but de construire le sens d'un texte, et ce, souvent sans en être pleinement conscients (Pressley et McCormick, 1995). Ils font preuve de flexibilité et utilisent des stratégies différentes selon le type de texte devant lequel ils sont placés (Giasson, 2011). Bien que certains élèves habiles développent seuls des stratégies de lecture efficaces, ce n'est pas le cas de tous. Ces stratégies doivent donc être enseignées et rendues explicites par les enseignants puisque les élèves peuvent difficilement y avoir accès autrement (Giasson, 2011; Topping et Ferguson, 2005).

Des enseignants des $2^{e}$ et $3^{e}$ cycles du primaire d'une commission scolaire de la région du Saguenay-Lac-Saint-Jean ont remarqué que plusieurs élèves faisaient usage d'un nombre limité de stratégies de lecture. C'est après avoir partagé cette préoccupation avec une conseillère pédagogique, qui a ensuite communiqué avec la chercheure, que cette démarche de recherche-action s'est amorcée.

Le constat formulé par les enseignants de cette commission scolaire peut être mis en relation avec les observations de Barclay, DeCleene et Juettner (2010) qui s'intéressent au fait que les élèves semblent bien connaitre les stratégies, mais qu'ils ne les utilisent pas. À ce propos, Giasson (2011) précise qu'« [ê]tre stratégique signifie beaucoup plus que connaitre les stratégies. Il faut savoir les combiner et les adapter à un plan global » (p. 26).

Cette situation rapportée par des enseignants du primaire trouve écho dans les écrits scientifiques qui affirment que, de manière générale, les enseignants accordent de l'importance aux stratégies de lecture, mais qu'ils se sentent parfois démunis devant la complexité de leur enseignement. De plus, Dobson Scharlach (2008) rapporte que certains enseignants trouvent exigeantes les démarches d'appropriation et de mise en œuvre des différents programmes d'enseignement des stratégies de lecture qui leur sont proposés. Par conséquent, la compréhension en lecture serait fréquemment évaluée, mais rarement enseignée (Gill, 2008).

Le présent article informe sur le contexte de réalisation de cette recherche-action qui s'intéresse à une problématique relative à l'enseignement des stratégies de lecture. Le cadre de référence situe le lecteur quant aux processus et stratégies mobilisés lors de la lecture ainsi qu'à certaines modalités pédagogiques telles que le matériel et les groupements d'élèves. La démarche méthodologique, quant à elle, précise les aspects spécifiques à la collecte des données. Enfin, les résultats sont présentés et discutés.

\section{Problématique}

Un enseignement efficace de stratégies de lecture implique que l'enseignant en ait une bonne connaissance, que les stratégies soient expliquées à voix haute et qu'elles soient utilisées devant et par les élèves dans des contextes réels et variés de lecture (Fielding et Pearson, 1994; Giasson, 2011; 
Routman, 2007). Ainsi, le simple fait de modéliser une stratégie n’assure pas son transfert par les élèves dans diverses situations (Fisher et coll., 2008). C'est donc par la modélisation et l'utilisation des stratégies dans des situations réelles de lecture que les élèves les acquièrent et accroissent leurs compétences (Fielding et Pearson, 1994; Gouvernement du Québec, 2005; Pardo, 2004). Comme le mentionnent certains auteurs, l'importance des stratégies de lecture et de leur enseignement a été abondamment documentée (Duffy, 1993; Taylor, Peterson, Pearson et Rodriguez, 2002). Or, malgré cela, certains enseignants trouvent difficile de mettre en œuvre une telle démarche d'enseignement (Fisher, 2002; Pressley et Afflerbach, 1995).

Ainsi, à la lumière du constat effectué par des enseignants du primaire et des résultats de différentes études (Fisher, 2002; Fisher, Frey et Lapp, 2008; Pardo, 2004; Pressley et Afflerbach, 1995), une recherche-action a été menée dans le but d'accompagner des enseignants dans la réflexion sur leurs pratiques et la mise à l'essai de nouvelles façons de faire relatives à l'enseignement des stratégies de lecture. De façon spécifique, cette étude poursuivait deux objectifs : 1- Identifier les stratégies de lecture enseignées par des enseignants des $2^{e}$ et $3^{e}$ cycles du primaire et leurs modalités d'enseignement et 2- Expérimenter des pistes d'amélioration des pratiques pédagogiques de ces enseignants en regard de l'enseignement de stratégies de lecture et réfléchir à leur mise en place.

Il importe de préciser que le second objectif a constitué la préoccupation principale de cette recherche. Or, l'identification des stratégies enseignées et de certaines modalités d'enseignement nous est apparue comme une démarche essentielle dont les résultats allaient servir d'ancrage à l'expérimentation de pistes d'amélioration de pratiques relatives à l'enseignement de stratégies de lecture. Ainsi, le présent article rend compte des résultats relatifs au premier objectif de la recherche.

\section{Cadre de référence}

Puisque le présent article vise à identifier les stratégies de lecture enseignées par des enseignants des $2^{\mathrm{e}}$ et $3^{\mathrm{e}}$ cycles du primaire ainsi que leurs modalités d'enseignement, le cadre de référence s'attarde principalement aux processus et aux stratégies de lecture. Cependant, il sera également question du matériel pédagogique et des groupements d'élèves.

\section{Modèle interactif de la compréhension en lecture}

La lecture est un processus interactif qui implique la relation entre trois variables : le lecteur, le texte et le contexte (Giasson, 1990; 2003). Ainsi, plus grande est la relation entre les variables, meilleure sera la compréhension d'un texte.Figure 1 : Modèle interactif de la compréhension en lecture 
Figure 1 : Modèle interactif de la compréhension en lecture

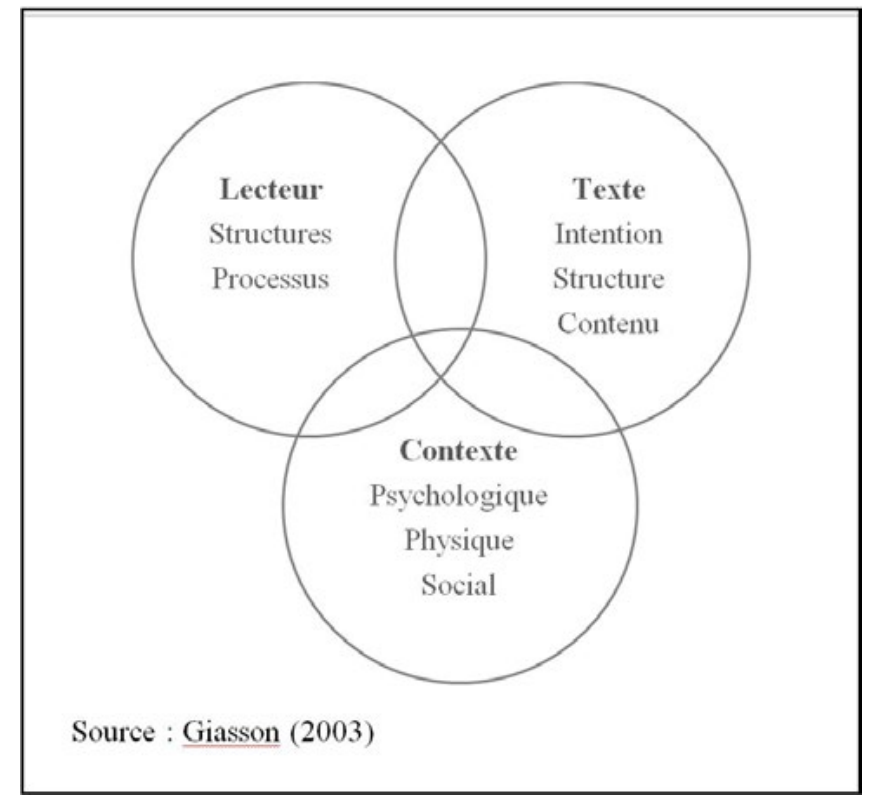

Comme l'explique Giasson (2003), le lecteur aborde le texte avec ses structures affectives et cognitives qui font référence à ses connaissances du monde et de la langue. Quant aux processus, ils renvoient aux stratégies déployées par le lecteur durant la lecture afin d'assurer la compréhension. Le lecteur est placé devant un texte qui reflète l'intention de l'auteur et dont le contenu est organisé selon le type de texte et la structure privilégiée. Quant au contexte, il situe le lecteur aux plans psychologique, physique et social. Le contexte psychologique renvoie à sa manière d'aborder le texte. Le contexte physique fait référence au lieu où se trouve le lecteur lors de la réalisation de la tâche, alors que le contexte social considère les interactions ainsi que les interventions des pairs et de l'enseignant. La compréhension d'un texte dépend donc de l'interaction entre les trois variables. Ainsi, un lecteur ayant développé peu de stratégies de lecture ou qui les gère avec difficulté verra sa compréhension affectée, et ce, malgré le fait que le texte puisse convenir à son niveau de compétence et qu'il soit placé dans un contexte favorable à la lecture. Il importe donc que les stratégies de lecture soient enseignées dans des contextes favorables afin que les élèves deviennent des lecteurs de plus en plus compétents (Pardo, 2004).

\section{Processus et stratégies de lecture}

La lecture est une activité complexe mettant à contribution de nombreux processus cognitifs regroupés selon cinq types : les microprocessus, les processus d'intégration, les macroprocessus, les processus d'élaboration ainsi que les processus métacognitifs (Giasson, 1990; Irwin, 2007). De façon générale, les microprocessus permettent la compréhension des informations contenues dans une 
phrase en se servant, par exemple, du contexte et des indices morphologiques (préfixe, suffixe) des mots. Quant aux processus d'intégration, ils contribuent à l'établissement de liens entre les phrases en ayant recours, entre autres, aux référents et aux connecteurs. En ce qui a trait aux macroprocessus, ils assurent une compréhension globale du texte en considérant sa structure et l'identification des idées principales. Les processus d'élaboration, pour leur part, permettent notamment de faire des inférences en établissant des liens entre le contenu du texte et les connaissances antérieures du lecteur. Enfin, les processus métacognitifs servent à la gestion de la compréhension.

Ces processus se traduisent en de nombreuses stratégies que le lecteur mobilise avant, pendant et après la lecture afin d'assurer la compréhension. Les stratégies utilisées avant de commencer la lecture permettent au lecteur de se préparer à aborder le texte, de planifier la tâche. Celles déployées pendant la lecture favorisent la formulation d'hypothèses ainsi que les ajustements nécessaires à la compréhension. Après la lecture, le lecteur revient sur l'ensemble du texte pour s'assurer qu'il a bien compris (Bédard et Montpetit, 2002; Giasson, 1995; Giasson, 2003; Gill, 2008; Gouvernement du Québec, 2001; Thériault et Nadeau-Tremblay, 2011). Inspirées des écrits de ces différents auteurs, nous avons répertorié, à la figure 2, des stratégies utilisées par le lecteur avant, pendant et après la lecture.

Figure 2 : Stratégies mobilisées lors d'une tâche de lecture

\begin{tabular}{l}
\hline AVANT la lecture \\
\hline - Lire le titre et les sous- \\
titres \\
- Regarder les illustrations \\
- Déterminer une intention \\
de lecture \\
- Survoler le texte \\
-Activer les connaissances \\
antérieures en lien avec le \\
contenu du texte \\
- Formuler des hypothèses
\end{tabular}

\begin{tabular}{l}
\hline PENDANT la lecture \\
\hline - S'assurer du bon \\
déroulement en se servant \\
des indices sémantiques et \\
syntaxiques : poursuivre la \\
lecture ou revenir en \\
arrière pour découvrir le \\
sens d'un mot nouveau, \\
découper un mot en \\
syllabes pour en faciliter \\
lidentification, penser à \\
des mots de meme famille, \\
se servir de la ponctuation, \\
se référer aux connecteurs, \\
faire de petits résumés, \\
explorer la structure dú \\
texte, relire les sous-titres, \\
regarder les illustrations, \\
faire des inférences, \\
formuler des hypothèses, \\
etc.
\end{tabular}

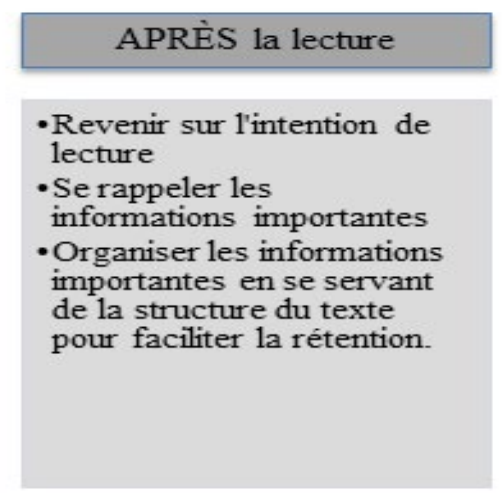

Alors qu'au premier cycle, l'élève acquiert des stratégies de reconnaissance et d'identification des mots parce qu'il est généralement familier avec le contenu des textes proposés, à partir du deuxième cycle, son répertoire de stratégies se diversifie, car les tâches de lecture se complexifient. Les textes sont alors plus longs, le contenu plus dense et les genres littéraires variés. La compréhension exigera donc du lecteur qu'il gère efficacement un ensemble de stratégies. Au troisième cycle, s'ajoute à 
cela le fait que l'élève devient de plus en plus conscient de l'efficacité des stratégies (Giasson, 2003; Gouvernement du Québec, 2001).

Une gestion efficace de la compréhension requiert que le lecteur soit en mesure d'effectuer de nombreux ajustements en cours de lecture selon les besoins de la situation (Dobson Scharlach, 2008). Ainsi, comme l'explique Giasson (2003), la lecture est un processus holistique exigeant du lecteur la gestion d'un ensemble de processus et, par conséquent, les stratégies de lecture ne peuvent être enseignées ni apprises d'une manière isolée et hiérarchisée.

\section{Matériel pédagogique}

Pour Legendre (2005), le matériel pédagogique ou didactique constitue le support qui facilite l'enseignement et l'apprentissage. Nadeau-Tremblay (2008) fait remarquer que Giasson (2003) parle plutôt de supports pédagogiques qu'elle regroupe selon cinq catégories : les manuels de lecture, les manuels des différentes matières, les livres de littérature jeunesse, les journaux ainsi que les supports électroniques et virtuels. À ce matériel, Nadeau-Tremblay (2008) ajoute celui élaboré par l'enseignant. Malgré la diversité du matériel accessible, Spallanzani, Lebrun, Biron, Lenoir, Roy, Larose et coll. (2001) observent que les enseignants se tournent principalement vers les manuels de lecture pour l'enseignement des stratégies de lecture, et ce, bien qu'il soit souhaitable que les élèves aient accès à du matériel varié, adapté à leurs besoins et stimulant pour développer la compétence à lire (Nadeau-Tremblay, 2008; Pardo, 2004).

\section{Groupements des élèves}

Les activités quotidiennes d'une classe invitent à la diversité et à la flexibilité des groupements des élèves, qui devraient être prévus en fonction des objectifs que poursuit l'enseignant (Hattie, 2002). Giasson (2003) identifie trois principaux types de groupements : le groupe-classe, les sous-groupes et le travail individuel. Selon cette auteure, l'intervention auprès du groupe-classe est tout indiquée au début et au terme d'une activité de lecture puisqu'il favorise le partage d'une expérience commune. Les sous-groupes, quant à eux, favorisent la collaboration entre les pairs et peuvent être hétérogènes ou homogènes (Nadeau-Tremblay, 2008). Dans tous les cas, il revient à l'enseignant de déterminer les types de groupements des élèves en fonction des besoins puisqu'il s'agit là d'un facteur influençant l'engagement des élèves (Ediger, 2002; Hattie, 2002; Nadeau-Tremblay, 2008). 


\section{Démarche méthodologique privilégiée}

Cette troisième section rend compte de l'opérationnalisation du premier objectif de la recherche. Toutefois, il sera question du type de démarche de recherche privilégié pour l'ensemble de l'étude, du profil des participants, du déroulement de la collecte des données spécifique au premier objectif ainsi que des outils qui lui ont été nécessaires.

\section{Type de recherche}

Le besoin manifesté par des enseignants de réfléchir à l'amélioration de leurs pratiques pédagogiques a conduit à une démarche de recherche qualitative de type recherche-action (Couture, Bednarz et Barry, 2007). Comme l'expliquent Dolbec et Clément (2004), en recherche-action, la participation active des enseignants est requise puisque c'est en intégrant leurs pratiques au processus de recherche que les changements pourront être apportés. À l'instar de ces mêmes auteurs, le processus cyclique de la recherche-action a permis de collecter des données lors de trois journées de développement professionnel, de prendre du recul pour les analyser et de comprendre les pratiques des enseignants pour ensuite leur présenter les résultats et apporter des ajustements à leurs pratiques. L'ouverture de la recherche qualitative a favorisé l'étude de réalités diverses, construites de multiples façons, mais tout aussi valides (Denzin et Lincoln, 2003).

\section{Profil des participants}

Douze enseignantes, un enseignant du primaire et deux conseillères pédagogiques d'une même commission scolaire ainsi que la chercheure ont travaillé de concert à la résolution de la situation problématique identifiée par les acteurs du milieu scolaire. Le tableau 1 informe sur les caractéristiques des participants.

Tableau 1 : Caractéristiques des enseignants

\begin{tabular}{|c|c|c|c|c|c|}
\hline \multicolumn{2}{|c|}{$\begin{array}{c}\text { Répartition des enseignants selon les } \\
\text { niveaux scolaires }\end{array}$} & \multirow{2}{*}{\begin{tabular}{|c|}
$\begin{array}{c}\text { Années } \\
\text { d'expérience à ce } \\
\text { niveau }\end{array}$ \\
$0-4$ années
\end{tabular}} & \multirow{2}{*}{\begin{tabular}{|c|}
$\begin{array}{c}\text { Nombre } \\
\text { d'enseignants }\end{array}$ \\
7 enseignantes
\end{tabular}} & \multirow{2}{*}{\begin{tabular}{|c|c|}
$\begin{array}{c}\text { Années } \\
\text { d'expérience en } \\
\text { enseignement }\end{array}$ \\
$6-10$ années
\end{tabular}} & \multirow{2}{*}{\begin{tabular}{|l|}
\multicolumn{1}{|c|}{$\begin{array}{c}\text { Nombre } \\
\text { d'enseignants }\end{array}$} \\
4 enseignantes \\
1 enseignant
\end{tabular}} \\
\hline $3^{e}$ année & $\begin{array}{l}2 \text { enseignantes } \\
1 \text { enseignant }\end{array}$ & & & & \\
\hline $4^{e}$ année & 4 enseignantes & 5 - 9 années & 4 enseignantes & 11 - 15 années & 2 enseignantes \\
\hline $3^{e}$ et $4^{e}$ années & 4 enseignantes & 10 années et + & 2 enseignantes & 16 - 20 années & 2 enseignantes \\
\hline $4^{e}$ et $5^{e}$ années & 1 enseignante & & & 21 - 25 années & 3 enseignantes \\
\hline $5^{e}$ année & 1 enseignante & & & 26 années et + & 1 enseignante \\
\hline
\end{tabular}


Dans le contexte de cette recherche, on remarque que les enseignants sont majoritairement responsables de classe de $2^{\mathrm{e}}$ cycle et qu'un peu plus du tiers sont au sortir de la période d'insertion professionnelle.

\section{Collecte et analyse des données}

D’entrée de jeu, il importe de rappeler qu'il s'agit ici de la collecte des données relatives au premier objectif de la recherche ainsi formulé : identifier les stratégies de lecture enseignées par des enseignants des $2^{e}$ et $3^{e}$ cycles du primaire et leurs modalités d'enseignement. Ces données ont été obtenues par l'entremise d'un questionnaire auquel les treize enseignants ont répondu individuellement lors d'une première journée de développement professionnel. Cette démarche auprès des enseignants nous est apparue essentielle à mener puisqu'elle allait permettre ultérieurement de travailler à partir du contexte réel de pratique des participants, ce qui sied à la dynamique d'amélioration préconisée par la recherche-action.

Ce questionnaire était constitué de questions ouvertes afin de ne pas induire les réponses des participants et de favoriser la diversité des informations collectées. L'aspect qualitatif du questionnaire visait donc la qualité de l'information à collecter davantage que la quantité (Gravel et Beaudin, 1994). Les questions ont été regroupées selon trois sections. Une première section demandait aux enseignants d'identifier les stratégies utiles enseignées dans leur classe avant, pendant et après la lecture. La seconde section les interrogeait sur le matériel utilisé et les groupements d'élèves pour cet enseignement. Quant à la troisième section, elle s'intéressait aux forces des enseignants et aux difficultés rencontrées lors de l'enseignement des stratégies de lecture. Mentionnons que seuls les résultats concernant les deux premières sections du questionnaire sont considérés dans cet article.

Quant au traitement du contenu des questionnaires, les éléments présentés dans le cadre de référence ont été mis à contribution pour l'analyse des stratégies énoncées par les enseignants, du matériel pédagogique utilisé et des groupements des élèves privilégiés. Le travail d'analyse a été effectué par une assistante de recherche et la chercheure. Enfin, les résultats ont été présentés aux enseignants qui ont pu les commenter et les valider.

\section{Résultats}

Cette section présente les résultats concernant les stratégies utiles avant, pendant et après la lecture que des enseignants des $2^{e}$ et $3^{e}$ cycles affirment enseigner aux élèves. Il sera également question des moyens mis en place tels que le matériel pédagogique utilisé ainsi que les groupements d'élèves privilégiés pour cet enseignement. 


\section{Portrait des stratégies de lecture enseignées}

Les trois tableaux qui suivent dressent le portrait des stratégies enseignées à chacun des trois temps de la lecture dans la classe des participants. Ainsi, le tableau 2 informe sur les stratégies utiles qui sont enseignées par les enseignants avant la lecture. Les stratégies utiles qui sont abordées par les enseignants pendant la lecture sont rapportées dans le tableau 3, alors que le tableau 4 s'intéresse aux stratégies enseignées après la lecture.

Tableau 2 : Stratégies utiles qui sont enseignées AVANT la lecture

\begin{tabular}{|c|c|}
\hline Stratégies AVANT la lecture & Occurrences \\
\hline Discuter à propos des illustrations & 10 \\
\hline Lire le titre et les sous-titres & 9 \\
\hline Activer les connaissances des élèves & 9 \\
\hline Déterminer le type de texte & 8 \\
\hline Préciser une intention de lecture & 7 \\
\hline Anticiper, prédire et formuler des hypothèses & 6 \\
\hline Survoler le texte & 4 \\
\hline S'attarder au texte (longueur et nombre de paragraphes) & 2 \\
\hline Connaitre l'intention de l'auteur & 1 \\
\hline Expliquer la tâche & 1 \\
\hline
\end{tabular}

Le tableau 2 indique que dix stratégies utiles AVANT la lecture sont enseignées par les enseignants pour un total de 58 occurrences. Discuter à propos des illustrations, lire le titre et les sous-titres, activer les connaissances antérieures, anticiper le contenu et formuler des hypothèses sont autant de stratégies qui permettent au lecteur d’aborder le texte en étant bien préparé. Lors de la présentation des résultats aux enseignants, ils ont mentionné que les stratégies utiles AVANT la lecture sont les plus abordées avec les élèves parce qu'ils les considèrent plus faciles à enseigner que celles utiles PENDANT et APRĖS la lecture. Ils ont expliqué ce phénomène par le fait qu'ils sont davantage conscients de ces stratégies et qu'il serait plus aisé de les utiliser devant les élèves. 
Tableau 3 : Stratégies utiles qui sont enseignées PENDANT la lecture

Stratégies PENDANT la lecture

Occurrences

Utiliser le contexte (mot avant ou après) pour identifier ou comprendre un mot

8

Vérifier la perte de compréhension

Recourir aux référents ainsi qu'aux mots de même famille pour comprendre le sens d'un mot

Recourir au découpage syllabique

Formuler des hypothèses et les vérifier

Surligner les informations importantes

Faire des pauses pour se remémorer l'intention de lecture

Chercher des mots difficiles dans le texte

5

4

3

3

2

1

Quant au tableau 3, il montre que huit stratégies mobilisées PENDANT la lecture seraient enseignées aux élèves pour un total de 27 occurrences. Il semble que les stratégies principalement enseignées soient les suivantes : a- Utiliser le contexte (mot avant ou après) pour identifier ou comprendre un mot; b-Recourir aux référents ainsi qu'aux mots de même famille pour comprendre le sens d'un mot et c- Recourir au découpage syllabique. II s’agit de stratégies adéquates pour identifier un mot ou pour en assurer sa compréhension. Cependant, on constate que chaque stratégie compte peu d'occurrences à l'exception de celle liée à l'utilisation du contexte pour l'identification ou la compréhension d'un mot, qui en dénombre huit. Enfin, «Vérifier la perte de compréhension » est une stratégie rapportée par cinq enseignants qui n’ont pas été plus explicites quant aux stratégies enseignées qui permettent cette vérification.

Ces stratégies rapportées par les enseignants permettent principalement de comprendre l'information contenue dans une phrase et relèvent des microprocessus. Or, rappelons qu'à compter du deuxième cycle du primaire, les élèves sont exposés à des textes dont le contenu est de plus en plus complexe. Ils devraient donc être amenés à développer des stratégies facilitant une compréhension plus globale du texte, comme le recours aux processus d'intégration, qui permettent d'établir des liens entre les phrases. Lors de la discussion qui a suivi la présentation des résultats, les enseignants ont expliqué qu'ils trouvent difficile l'enseignement de telles stratégies. Ils ont également mentionné qu'ils tiennent parfois pour acquis que les élèves plus vieux connaissent ces stratégies et que, par conséquent, ils ont tendance à accorder peu de temps à leur enseignement explicite. 
Tableau 4 : Stratégies utiles qui sont enseignées APRÈS la lecture

Stratégies APRÈS la lecture

Occurrences

Partager l'appréciation du texte

Revenir sur l'intention de lecture

Surligner les informations importantes

Faire un retour sur ce qui a été lu

Résumer ce qui a été lu

Revenir sur les hypothèses

Répondre à des questions sur le texte

Revoir la structure du texte

Surligner, dans les questions, les indices aidant à y répondre

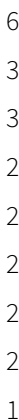

Neuf stratégies utiles APRÈS la lecture sont rapportées par les enseignants. Cependant, l'énoncé comptant le plus grand nombre d'occurrences n'est pas une stratégie facilitant la compréhension puisqu'il s'agit de partager son appréciation du texte. Ce sont donc huit stratégies qui sont enseignées pour un total de 17 occurrences. Ainsi, revenir sur l'intention de lecture, surligner les informations importantes, faire un retour sur ce qui a été lu, sont autant de stratégies qui permettent de mettre un terme à la lecture en s'assurant d'avoir bien compris. Or, il est constaté que chaque stratégie compte très peu d'occurrences, ce qui révèle que les stratégies utiles après la lecture seraient peu enseignées par ces participants. Ce constat a été confirmé par les enseignants qui ont confié être moins familiers avec de telles stratégies. Ils ont également expliqué que leurs interventions après la lecture visent principalement à supporter les élèves lors de la réalisation de tâches de compréhension de texte.

La figure 3 illustre la répartition des stratégies enseignées avant, pendant et après la lecture.

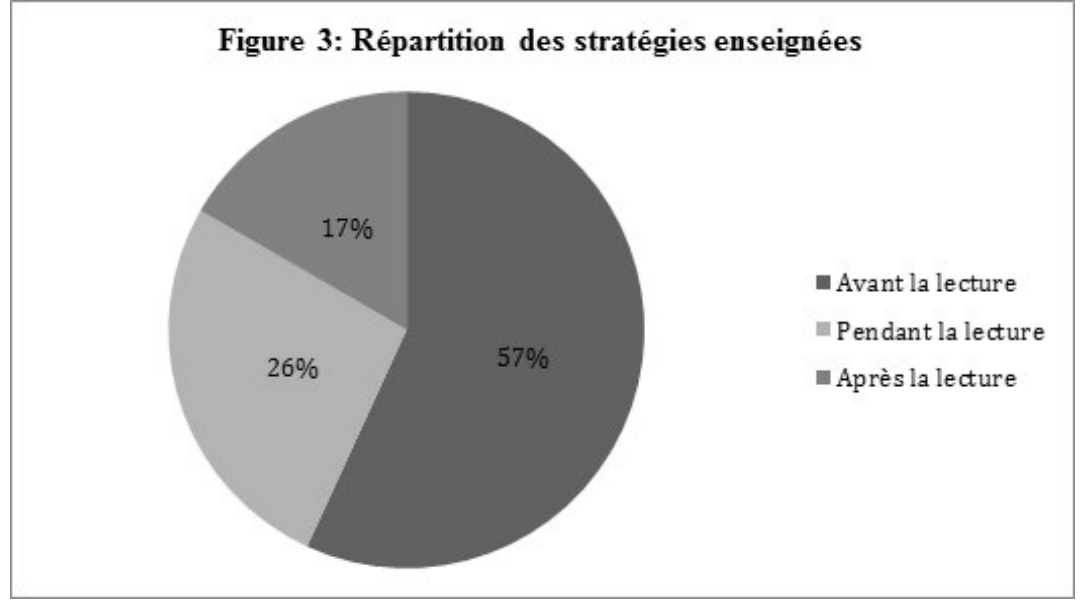


Finalement, cette répartition des stratégies met en évidence le fait que ce sont celles utilisées avant la lecture qui sont les plus enseignées par ces enseignants, suivies des stratégies nécessaires pendant et après la lecture. La présentation des résultats et les échanges avec les enseignants ont contribué à une prise de conscience de leur part quant à la place accordée aux stratégies de lecture et à leur enseignement, ce qui leur a permis d'apporter des ajustements nécessaires à leurs interventions.

\section{Matériel pédagogique et groupements des élèves}

Après avoir été questionnés à propos des stratégies de lecture enseignées en classe, les enseignants ont été invités à s'exprimer quant au matériel pédagogique utilisé ainsi qu'à propos des groupements d'élèves préconisés pour les enseigner. Cette section présente donc les résultats relatifs à ces deux aspects.

\section{Matériel pédagogique}

Les résultats indiquent que 11 enseignants sur 13 se servent des manuels scolaires pour l'enseignement des stratégies de lecture. Ces manuels font partie d'ensembles didactiques élaborés par différentes maisons d'édition. De plus, 12 enseignants sur 13 révèlent avoir également recours à du matériel divers, et la répartition de cette utilisation est la suivante: les journaux et les romans jeunesse ont été mentionnés par trois enseignants, les Cliniques de lecture sont utilisées par deux enseignants tout comme les textes du domaine «Éthique et culture religieuse ». Le magazine «Les Explorateurs », des livres de littérature jeunesse, un site Internet sur l'Univers social ont tous été mentionnés par un enseignant. Enfin, il appert que le matériel didactique demeure la principale ressource pédagogique pour enseigner les stratégies de lecture.

\section{Groupements des élèves}

En ce qui a trait aux groupements des élèves privilégiés par les enseignants pour l'enseignement des stratégies de lecture, les résultats indiquent que l'intervention auprès de l'ensemble du groupeclasse constitue un type de groupement d'élèves considéré dans une forte proportion puisque neuf enseignants sur 13 affirment y recourir. Par ailleurs, ils ne sont que trois enseignants sur 13 à faire usage de l'intervention individuelle, alors que deux enseignants forment des sous-groupes pour enseigner des stratégies de lecture en fonction des besoins des élèves. 


\section{Discussion}

Les résultats sont maintenant discutés au regard des stratégies de lecture enseignées, du matériel pédagogique utilisé et des groupements d'élèves formés.

\section{Portrait des stratégies de lecture enseignées}

Les données obtenues auprès des treize enseignants indiquent que ce sont les stratégies mobilisées avant la lecture qui sont les plus enseignées, suivies des stratégies utiles pendant et après la lecture.

Selon les résultats, les stratégies enseignées avant la lecture sont celles réputées pour bien préparer à la tâche. Il semble que les stratégies permettant l'identification ou la compréhension d'un mot soient les plus travaillées pendant la lecture. Alors que les textes se complexifient aux $2^{e}$ et $3^{e}$ cycles, il semble que peu de stratégies de gestion de la compréhension mobilisées pendant la lecture seraient enseignées aux élèves. Les stratégies utiles après la lecture sont adéquates, mais chacune d'entre elles compte très peu d'occurrences. Les résultats révèlent donc que, dans l'ensemble, peu de stratégies de lecture sont enseignées.

Ces résultats divergent de ceux obtenus par Martel et Lévesque (2010) lors d'une enquête menée auprès d'enseignants des $2^{e}$ et $3^{e}$ cycles du primaire. Selon les résultats de ces chercheurs, « [p]resque la totalité des stratégies de compréhension en lecture identifiées dans le Programme de formation de l'école québécoise sont travaillées par plus de la moitié des enseignants » (Martel et Lévesque, 2010, p. 43). Contrairement au questionnaire constitué d'une échelle de Likert utilisé dans le cadre de cette enquête, notre outil était composé de questions ouvertes. Ainsi, cette différence au plan méthodologique et le fait que bon nombre de lecteurs habiles ne réalisent pas toutes les stratégies qu'ils mobilisent lors de la lecture, incluant les enseignants (Pressley et McCormick, 1995), peuvent apporter des éléments expliquant les résultats de notre étude.

Tout comme l'ont constaté Martel et Lévesque (2010), les enseignants trouvent difficile d'expliquer et de modéliser les stratégies de lecture, ce qui contribuerait à expliquer ces résultats. Le fait que certaines stratégies de lecture soient peu connues des enseignants est un autre facteur à considérer.

Une autre piste d'explication des résultats se situe par rapport à l'utilisation du matériel didactique. Bien que cet aspect soit discuté à la section suivante, mentionnons tout de même que ce type de matériel constitue la principale ressource utilisée pour l'enseignement des stratégies de lecture. Il semble que les enseignants aient tendance à considérer le matériel didactique comme une ressource fiable (Vargas, 2006). Or, il est possible que celui-ci n’aborde qu'un nombre limité de stratégies. Comme le mentionne Gill (2008), un enseignement efficace de la compréhension en lecture exige que les enseignants connaissent bien l'ensemble des stratégies et c'est en ce sens que s'est poursuivie la recherche. 


\section{Matériel pédagogique}

En ce qui a trait au matériel utilisé pour l'enseignement des stratégies de lecture, les résultats indiquent que les manuels scolaires constituent le principal outil dont se servent les enseignants. Ce résultat ne surprend pas puisque Spallanzani et coll. (2001) observent que les manuels scolaires constituent le matériel le plus utilisé dans les classes. Bien que les manuels fassent l'objet de nombreuses critiques, Giasson (2003) remarque toutefois que leur contenu a évolué au fil du temps et qu'ils sont désormais plus attrayants, qu'ils véhiculent moins de stéréotypes, que les guides pédagogiques considèrent davantage les résultats de recherche et que les activités proposées sont plus signifiantes. Lors de la présentation des résultats, des enseignants confiaient ne pas toujours apprécier les textes présentés dans les manuels et qu'ils les trouvaient même ennuyants à certains moments, mais qu'ils se sentaient tout de même dans l'obligation d'utiliser ce matériel. Les propos de ces enseignants confirment ceux de Giasson (2003) qui affirme que : " [l]a très grande majorité des enseignants utilisent un manuel, même s'ils reconnaissent que ce n'est pas toujours la meilleure façon d'enseigner la lecture» (p. 105). Par ailleurs, comme le suggèrent certains auteurs (Giasson, 2003; Nadeau-Tremblay, 2008; Pardo, 2004), quelques enseignants ont également recours à d'autres types de matériel pour enseigner les stratégies de lecture.

Ainsi, il semble que les manuels scolaires exercent une forte influence sur les pratiques des enseignants (Lenoir, 2006). Cependant, comme le soulignent Allington et McGill-Franzen (2000), il ne s'agit pas ici de condamner le matériel didactique, mais plutôt de se pencher sur l'utilisation qui en est faite. II s'agit là d'un point de vue que partage Routman (2007) en véhiculant l'idée que le matériel didactique devrait être envisagé comme une ressource pédagogique plutôt qu'un guide à suivre.

\section{Groupements des élèves}

En ce qui a trait aux groupements des élèves, les résultats indiquent que le groupe-classe constitue le mode de regroupement privilégié par les participants pour l'enseignement des stratégies de lecture. Cette pratique correspond aux observations réalisées dans des classes dans le cadre d'une recherche menée par Taylor et coll. (2002). Or, tandis que Giasson (2003) affirme qu'il s'agit là d'un regroupement particulièrement intéressant pour l'enseignement des stratégies de lecture, d'autres chercheurs comme Taylor, Pearson, Clark et Walpole (2000) remarquent que les enseignants les plus efficaces par rapport à l'enseignement de la lecture privilégient les interventions en sous-groupes.

Enfin, bien que l'enseignement au groupe-classe soit une pratique reconnue, il semble que les enseignants gagneraient à regrouper davantage les élèves en sous-groupes pour l'enseignement des stratégies de lecture. D'ailleurs, seulement deux enseignants ont affirmé qu'ils constituaient des sous-groupes selon les besoins des élèves et trois d'entre eux ont mentionné qu'ils faisaient certaines interventions sur une base individuelle lorsque cela s'avérait nécessaire. 


\section{Originalité et limites de la recherche}

Comme le souligne Duffy (1993), de nombreux programmes d'enseignement de la lecture ont été expérimentés au fil des ans. De tels programmes se centrent sur l'expérimentation d'interventions spécifiques dans le cadre de devis où des comparaisons sont établies entre des groupes expérimentaux et contrôles. Cet auteur mentionne également l'importance de s'intéresser davantage à des démarches de recherche plus écologiques, c'est-à-dire en tenant compte du contexte réel (possibilités et contraintes) qui prévaut dans les classes. Ainsi, le fait que la problématique de cette étude ait émergé du milieu scolaire et qu'il s'agisse d'une démarche de recherche-action contribue à l'originalité de la présente étude puisque, comme le mentionne Purcell-Gates (2000), le domaine de recherche de la lecture ne doit pas se limiter à mettre en œuvre des devis expérimentaux ou quasi expérimentaux comme cela est souvent le cas. En effet, des démarches de recherches qualitatives sont nécessaires à la compréhension de certains phénomènes de ce domaine. La prise en compte des besoins exprimés par les enseignants est d'autant plus pertinente. En effet, selon Desgagné, Bednarz, Couture, Poirier et Lebuis (2001), il est nécessaire de tenir compte de la perspective des enseignants dans le but, d'une part, de mieux cerner des éléments de contexte de la classe et, d'autre part, de faire en sorte que la participation à une recherche constitue aussi une occasion de développement professionnel viable pour les enseignants qui y prennent part.

En ce qui a trait aux limites de l'étude, une première d'entre elles se situe au plan méthodologique puisqu'il s'agit de pratiques déclarées par les enseignants. Cependant, Topping et Ferguson (2005) constatent qu'il s'agit d'une des principales méthodes utilisées pour collecter des données dans le domaine de la recherche en lecture. Une piste de recherche complémentaire pourrait consister à travailler l'amélioration des pratiques dans une perspective analytique à partir de séquences vidéos. Une seconde limite vient du savoir produit puisque, comme l'explique Savoie-Zajc (2000), les résultats engendrent des savoirs contextuels influencés par les milieux de vie des enseignants. Dans notre contexte, cette limite a eu ses avantages du fait que les résultats obtenus ont constitué une première étape importante permettant d'ancrer la dynamique de changement de la recherche-action à même les pratiques des participants. Enfin, une troisième limite concerne le nombre restreint de participants qui ne permet pas la généralisation des résultats. 


\section{Conclusion}

Cet article a fait état d'une partie des résultats obtenus dans le cadre d'une recherche-action qui a été menée avec treize enseignants des $2^{e}$ et $3^{e}$ cycles du primaire désireux d'améliorer leurs pratiques relatives à l'enseignement des stratégies de lecture. Il s'est attardé à dresser un portrait des stratégies enseignées, du matériel utilisé ainsi que des groupements privilégiés. Les résultats révèlent que ce sont les stratégies utilisées avant la lecture qui sont enseignées en plus grand nombre, que les enseignants privilégient l'intervention auprès de l'ensemble du groupe-classe et que les stratégies sont principalement enseignées à l'aide des manuels scolaires. À la suite de ces premiers constats, les treize enseignants se sont fixé des défis professionnels au regard de l'enseignement des stratégies de lecture et c'est en ce sens que la recherche s'est poursuivie.

La réalisation de ce projet a été rendue possible grâce au soutien financier de la Commission scolaire du Pays-des-Bleuets et du Consortium régional de recherche en éducation. 


\section{Références}

Allington, R. et McGill-Franzen, A. (2000). Looking back, looking forward : A conversation about teaching reading in the $21^{\text {st }}$ century. Reading Research Quarterly, 35(1), 136-153.

Barclay, M., DeCleene, J. et Juettner, M. R. (2010). Caution! Hard Hat Area! Comprehension Under Construction : Cementing a Foundation of Comprehension Strategy Usage That Carries Over to Independant Practice. The Reading Teacher, 63(8), 687-691.

Bédard, D. et Montpetit, D. (2002). Stratégies... Stratégies... Pour une lecture efficace au primaire. Anjou : Les éditions CEC inc.

Couture, C. (2011). Accompagner le personnel enseignant dans le développement de sa pratique éducative en science et technologie. Dans G. Samson, A. Hasni, D. Gauthier et P. Potvin (dir.), Pour une collaboration école-université en science et techno: Des pistes pour l'apprentissage (p. 51-64). Québec: Presses de l’Université du Québec.

Couture, C., Bednarz, N. et Barry, S. (2007). Conclusion : Multiples regards sur la recherche participative: une lecture transversale. Dans M. Anadon (dir.), La recherche participative: multiples regards (p. 205-221). Saint-Nicolas: Presses de l'Université du Québec.

Desgagné, S., Bednarz, N., Couture, C., Poirier, L. et Lebuis, P. (2001). L'approche collaborative de recherche en éducation : un nouveau rapport à établir entre recherche et formation. Revue des sciences de l'éducation, XXVII(1), 33-64.

Denzin, N. K. et Lincoln, Y. S. (2003). Strategies of qualitative inquiry. Thousand Oaks, California, Sage.

Dobson Scharlach, T. (2008). START Comprehending : Students and Teachers Actively Reading Text. The Reading Teacher, 62(1), 20-31.

Dolbec, A. et Clément, J. (2004). La recherche-action. Dans T. Karsenti et L. Savoie-Zajc (dir.), La recherche en éducation : étapes et approches (p.181-208). Sherbrooke : Éditions du CRP.

Duffy, G. G. (1993). Teachers' Progress toward Becoming Expert Strategy Teachers. The Elementary School Journal, 94(2), 109-120.

Ediger, M. (2002). Grouping and organizing for instruction in reading. Kansas : University of Kansas. (Service reproduction ERIC No ED 471 842).

Fielding, L. C. et Pearson, P. D. (1994). Reading Comprehension : What Works. Educational Leadership, 51(5), 62-67.

Fisher, R. (2002). Shared thinking; Metacognitive modelling in the literacy hour. Reading : Literacy and Language, 36(2), 63-67.

Fisher, D., Frey, N. et Lapp, D. (2008). Shared Readings : Modeling Comprehension, Vocabulary, Text Structures, and Text Features for Older Readers. The Reading Teacher, 61(7), 548-556.

Giasson, J. (1990). La compréhension en lecture. Boucherville: Gaëtan Morin éditeur. 
Giasson, J. (1995). Stratégies de lecture. Dans L. Saint-Laurent, J. Giasson, C. Simard, J. J., Dionne et É. Royer (dir.). Programme d'intervention auprès des élèves à risque. Une nouvelle option éducative (p. 93-102). Boucherville : Gaëtan Morin éditeur.

Giasson, J. (2003). La lecture : De la théorie à la pratique (2éd.). Boucherville: Gaëtan Morin éditeur.

Giasson, J. (2011). La lecture : Apprentissage et difficultés. Montréal : Gaëtan Morin éditeur.

Gill, S. R. (2008). The Comprehension Matrix: A Tool for Designing Comprehension Instruction. The Reading Teacher, 62(2), 106-113.

Gravel, R. J. et Beaudin, G. (1994). La méthodologie du questionnaire : guide à l'usage de l'enquêteur. Saint-Laurent, Québec: Bo-Pré.

Gouvernement du Québec (2001). Programme de formation de l'école québécoise. Éducation préscolaire. Enseignement primaire. Québec: Ministère de l'Éducation.

Gouvernement du Québec (2005). Apprendre à lire. Action concertée pour le soutien à la recherche en lecture. Québec: Ministère l'Éducation, du Loisir et du Sport.

Hattie, J. (2002). Classroom composition and peer effects. International Journal of Educational Research, 37, 449-481.

Irwin, J. (2007). Teaching Reading Comprehension Processes (3e éd.). Englewood, Hew Jersey: Prentice-Hall.

Legendre, R. (2005). Dictionnaire actuel de l'éducation (3éd.). Montréal : Guérin.

Lenoir, Y. (2006). Orientations épistémologiques du nouveau curriculum et contribution des manuels scolaires à leur mise en œuvre. Dans J. Lebrun, J. Bédard, A. Hasni et V. Grenon (dir.), Le matériel didactique et pédagogique: soutien à l'appropriation ou déterminant de l'intervention éducative (p. 13-22). Québec : Presses de l'Université Laval.

Martel, V. et Lévesque, J-Y. (2010). La compréhension en lecture aux deuxième et troisième cycles du primaire : regard sur les pratiques déclarées d'enseignement. Revue canadienne de linguistique appliquée, 13(2), 27-53. Repéré à www.cjal-rcla.ca.

Nadeau-Tremblay, S. (2008). Le développement de la compétence en lecture en classe multiâge au primaire : une analyse des pratiques pédagogiques. (Mémoire de maitrise inédit). Université du Québec à Chicoutimi.

Pardo, L. S. (2004). What every teacher needs to know about comprehension. The Reading Teacher, 58(3), 272-280.

Pressley, M. et Afflerbach, P. (1995). Verbal Protocols of Reading: The Nature of Constructively Responsive Reading. Hillsdale, NJ : Erlbaum. 
Pressley, M. et McCormick, C. (1995). Strategies and metacognitive regulation of strategies : Basic theory and research. Dans M. Pressley et C. McCormick (dir.), Advanced educational psychology for educators, researchers, and policy makers (p. 27-47). New York : Harper Collins College Publishers.

Purcell-Gates, V. (2000). The Role of Qualitative and Ethnographic Research in Educational Policy. Repéré à www.readingonline.org.

Routman, R. (2007). Enseigner la lecture : revenir à l'essentiel. Montréal : Chenelière éducation.

Savoie-Zajc, L. (2000). La recherche qualitative/interprétative en éducation. Dans T. Karsenti, et L. Savoie-Zajc (dir.), Introduction à la recherche en éducation (p. 171-198). Sherbrooke : Éditions du CRP.

Spallanzani, C., Lebrun, J., Biron, D., Lenoir, Y., Roy, G.-R., Larose, F. et Masselier, G. (2001). Le rôle du manuel scolaire dans les pratiques enseignantes au primaire. Sherbrooke : Éditions du CRP.

Taylor, B. M., Pearson, P. D., Clark, K. et Walpole, S. (2000). Effective schools and accomplished teachers: Lessons about primary grade reading instruction in low-income schools. Elementary School Journal, 101, 121-166.

Taylor, B. M., Peterson, D. S., Pearson, P. D. et Rodriguez, M. M. (2002). Looking Inside Classrooms: Reflecting on the "how » as well as the " what » in effective Reading instruction. The Reading Teacher, 56(3), 270-279.

Thériault, P. et Nadeau-Tremblay, S. (2011). Susciter le plaisir de lire et acquérir des stratégies de lecture en classe multiâge. Dans C. Couture et P. Thériault (dir.), La classe multiâge d'aujourd'hui. Enseigner et apprendre en classes cycle ou intercycle (p. 63-78). Anjou : Éditions CEC.

Topping, K. et Ferguson, N. (2005). Effective literacy teaching behaviors. Journal of Research in Reading, 28(2), 125-143.

Vargas, C. (2006). Les manuels scolaires : imperfections nécessaires, imperfections inhérentes et imperfections contingentes. Dans M. Lebrun (dir.), Le manuel scolaire. Un outil à multiples facettes (p. 12-35). Québec: Presse de l'Université du Québec. 
TITRE: RECHERCHE-ACTION-FORMATION AU PRÉSCOLAIRE ET AU $1{ }^{\text {ER }}$ CYCLE DU PRIMAIRE : PISTES MÉTHODOLOGIQUES

Auteur(s): LiZAnne Lafontaine, Ph.D., Université du QuÉBec en Outaouais (UQO)

PUBLICATION: LA RECHERCHE-ACTION ET LA RECHERCHE-DÉVELOPPEMENT AU SERVICE DE LA LITTÉRATIE.

PAGES: $41-67$

Directeurs: ISABelle Carignan, Marie-Christine Beaudry et Françoıs LAROSE ÉdiTEUR: LES ÉdITIONS DE L'UNIVERSITÉ DE SHERBROOKE, 2016.

ISBN: 978-2-7622-0354-7

URI: HTTP://HDL.HANDLE.NET/11143/8809

DOI: HTTP://DX.DOI.ORG/10.17118/11143/8809 


\title{
Recherche-action-formation au préscolaire et au $1^{\text {er }}$ cycle du primaire : pistes méthodologiques
}

\author{
Lizanne Lafontaine, Ph.D., Université du Québec en Outaouais (UQO)
}

Résumé : La recherche-action-formation est un type de recherche encore peu documenté. Pourtant, des programmes de subvention québécois font de plus en plus appel à celle-ci afin d'accompagner des enseignantes dans le renouvèlement de leurs pratiques. Notre contribution souhaite à la fois définir ce qu'est la recherche-action-formation et en présenter le désign méthodologique. Pour ce faire, nous illustrerons nos propos par la présentation de notre propre recherche-action-formation ayant comme objectif général d'offrir des séminaires de formation et de recherche en littératie, volets lecture et oral, à deux enseignantes du préscolaire, à neuf du $1^{\text {er }}$ cycle du primaire et à deux orthopédagogues afin qu'ils élaborent et expérimentent des situations d’apprentissage et d'évaluation (SAÉ) intégrant la littératie. Loin de nous l'idée de présenter une recherche exemplaire, mais plutôt de démontrer la rigueur, la souplesse et la complexité de ce type de recherche ainsi que sa grande pertinence sociale et scientifique en éducation.

Mots-clés : recherche-action-formation, désign méthodologique, séminaires de formation, outils de collecte de données, préscolaire et primaire. 


\section{Introduction}

En 2009, le ministère de l'Éducation, du Loisir et du Sport (MÉLS) lançait un appel de projets intitulé Programme de formation continue du personnel scolaire qui avait « pour finalité de soutenir les universités dans la conception, le déploiement et l'évaluation de projets de formation conçus en partenariat avec le milieu scolaire » et répondant aux [...] domaines jugés prioritaires et ayant un lien avec les plans d'action et les orientations du Ministère (MÉLS 2009, p. 1), dont l'amélioration du français écrit. Ces projets devaient dispenser des activités de formation continue, créditées ou non, de diverses formes, et pouvaient prendre la forme de recherches-formations (MÉLS 2009, p. 2). Nous avons répondu positivement à cet appel en proposant un projet de recherche-action-formation, en collaboration avec le directeur de trois petites écoles situées en milieu défavorisé ${ }^{1}$ de Saint-Jérôme et la Commission scolaire de la Rivière-du-Nord (CSRDN)². Ce projet, financé par ce programme de 2010 à 2012, avait pour objectif général d'offrir des séminaires de formation et de recherche en littératie, volets lecture et oral, à deux enseignantes du préscolaire, à neuf du $1^{\text {er }}$ cycle du primaire ${ }^{3}$ et à deux orthopédagogues afin qu'ils élaborent et expérimentent des situations d'apprentissage et d'évaluation (SAÉ) intégrant la littératie. Les volets lecture et oral ont été choisis dans le but de répondre aux besoins des élèves du préscolaire et du $1^{\text {er }}$ cycle des écoles ciblées.

Mais qu'est-ce que la recherche-action-formation? Comment une telle recherche réalisée en collaboration étroite avec le milieu scolaire peut-elle se réaliser? Quelles en sont les forces et les limites? Notre contribution se concentrera plutôt sur les éléments méthodologiques de ce type de recherche et non sur les résultats de notre projet. Nous débuterons par la présentation de notre idée de recherche, le partenariat créé avec le milieu scolaire et la problématique ayant orienté notre choix méthodologique. Suivront ensuite quelques considérations théoriques autour de la recherche-action-formation. Nos objectifs d'intervention et de recherche seront aussi présentés. Notre désign méthodologique sera explicité ainsi que nos outils de collecte de données et nos données documentaires. Nous terminerons par les avantages et les limites de ce type de recherche.

1. Éric Morissette est le directeur de ces trois établissements scolaires. L'école Saint-Joseph accueille 134 élèves du préscolaire à la $6^{e}$ année; l'école de la Durantaye, 147 élèves du préscolaire à la $6^{\text {e }}$ année et l'Unité d'intervention santé

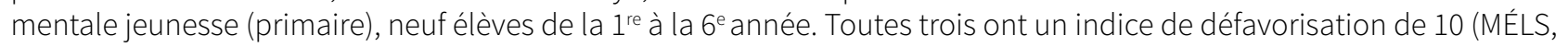
2011).

2. La CSRDN a payé pour la libération des enseignants tout au long de la recherche (2010-2012).

3. Trois des enseignantes sont des orthopédagogues enseignant dans des classes spéciales de communication. 


\section{Idée de recherche}

En tant que professeure-chercheuse en didactique du français et superviseure de stages sur le territoire de la Commission scolaire de la Rivière-du-Nord (CSRDN), nous avions pu constater l'intérêt d'enseignantes ${ }^{4}$ au regard d'une formation continue axée sur les apprentissages en français afin de mieux répondre aux besoins de leurs élèves et sur le renouvèlement de leurs pratiques pédagogiques, notamment au préscolaire et au $1^{\text {er }}$ cycle du primaire, lieux des premiers apprentissages. En effet, les enseignantes souhaitaient mettre en pratique des méthodes pédagogiques efficaces en littératie. Elles se questionnaient fortement sur l'enseignement et l'évaluation de l'oral et sur la mise en pratique d'activités de lecture répondant aux besoins de leurs élèves issus de milieux défavorisés. Elles désiraient aussi modifier leurs pratiques enseignantes afin de motiver davantage leurs élèves en lecture et en oral. De plus, nous étions intéressées à opérationnaliser dans le milieu scolaire certaines recommandations issues du Rapport du Comité d'experts sur l'apprentissage de l'écriture (MÉLS, 2008, p. 20), à savoir deux recommandations en particulier :

- la recommandation 7 invite le MÉLS à mettre [...] en place des mesures incitatives pour favoriser la formation continue en français des enseignantes et des enseignants du primaire [...] par rapport à des objets précis dont les suivants : [...] le contenu des programmes de formation, au sens de la compétence à écrire et des liens qu'elle peut établir avec la lecture et l'oral; les approches pédagogiques et didactiques; les approches à privilégier pour les élèves à besoins particuliers [...] notamment, dans le cas qui nous intéresse, les élèves issus de milieux défavorisés. Pour nous, la compétence à écrire englobe la lecture et l'écriture et a de forts liens avec l'oral, tous trois étant les volets du français enseignés à l'école;

- la recommandation 8 suggère aux universités de mettre [...] en place, en collaboration avec les services éducatifs des commissions scolaires et des établissements privés, des programmes souples de formation continue répondant aux besoins du milieu scolaire.

Nous en avons alors discuté avec le directeur de trois petites écoles situées en milieu défavorisé de Saint-Jérôme, et il nous est d'abord apparu qu'une formation continue organisée sous la forme de séminaires de deux jours chacun, répartis sur deux années scolaires (le séminaire est expliqué en détail dans la section 3), était une formule souple répondant à la fois aux besoins des enseignantes et aux exigences de la recommandation 8 du Rapport du Comité d'experts sur l'apprentissage de l'écriture. Ensuite, sur le plan conceptuel, le champ de la littératie, concept bien connu dans les écoles franco-canadiennes, était prometteur et répondait aux exigences de la recommandation 7 dudit rapport, notamment par rapport au lien entre lecture, écriture et oral ainsi qu'aux approches pédagogiques et didactiques à privilégier en milieu défavorisé. Nous partions donc d'un problème particulier rencontré par les enseignants dans leurs pratiques (Paillé, 1994; Marcel, 1999; Charlier, 2005; Lafortune, 2006; Prud'homme, Dolbec et Guay, 2011) répondant ainsi aux exigences de la recherche-action-formation, que nous décrirons dans la section 2. Nous avons également décidé

4. Nous avons travaillé avec une très grande majorité de femmes dans notre recherche-action-formation, c'est pourquoi nous avons privilégié l'emploi du féminin dans notre article. 
de faire appel à une orthopédagogue travaillant dans une des écoles ciblées, à trois conseillères pédagogiques de la CSRDN ${ }^{5}$ et à trois autres professeurs-chercheurs ${ }^{6}$ afin de constituer des formations de haut niveau en littératie et de toujours tenir compte des besoins de la CSRDN en matière de formation continue.

\section{Problématique}

Afin d'assurer la compréhension du lecteur, situons davantage ce qu'est la littératie et ses apports à notre projet ainsi que l'outil de planification pédagogique qu'est la SAÉ. Nous préciserons également les besoins du milieu d'accueil sur le plan de la formation en littératie.

\section{Littératie}

La littératie est une compétence générale qui permet à toute personne d'avoir accès au monde extérieur, d'interagir, de communiquer, d'apprendre, de socialiser et d'effectuer des calculs simples. Elle comprend les nombreuses formes de littératies requises pour réussir dans une économie basée sur le savoir comme la lecture, l'écriture, l'expression orale, le visionnement et la représentation (Grenier, Jones, Strucker, Murray, Gervais et Brink, 2008); elle constitue l'interface entre les compétences langagières et l'environnement culturel. Toutefois, l'apprentissage de la littératie va au-delà des habiletés en lecture et en écriture, elle est également un ensemble d'attitudes, de valeurs, d'habiletés et de comportements liés à la culture de l'écrit. Ainsi, ce concept illustre la manière dont une personne comprend et transmet des informations simples ou complexes dans des situations de communications orale et écrite, et dans une variété de supports imprimés ou non (Coghlan et Thériault, 2002).

De plus, c'est dans les milieux défavorisés, comme ceux des trois écoles ayant participé à notre recherche, que l'on retrouve le plus d'enfants qui éprouvent des difficultés à apprendre à lire (Brodeur, Dion, Laplante, Mercier, Desrochers et Bournot-Trites, 2011). Par exemple, dans les milieux socioéconomiques moyens/aisés, on estime le nombre d'heures de lecture faite aux enfants avant leur entrée à l'école entre 1000 et 1 700, alors que dans les milieux socioéconomiques défavorisés, cette estimation est de 25 heures. La fréquence des lectures faites aux enfants à la maison dès leur plus jeune âge est associée à leur rendement futur en lecture (Brodeur et coll., 2011). Également, les interactions adulte-enfant durant une lecture seraient positivement reliées au rendement en lecture au début de la scolarité (Brodeur et coll., 2011). Il est donc pertinent de penser que plusieurs élèves fréquentant les trois écoles ciblées dans notre recherche auraient été seulement exposés à 25 heures de lecture partagée à l'âge de 5 ans et aient par conséquent un risque élevé de difficulté en lecture.

5. Annie Desjardins, orthopédagogue à l'école Saint-Joseph; Julie Garnier, conseillère pédagogique à la réussite éducative, Isabelle Lowe, conseillère pédagogique en français au primaire, Karine Allard, conseillère pédagogique en langage, CSRDN.

6. Steve Bissonnette, Martine Leclerc et André C. Moreau, Université du Québec en Outaouais. 
Et ce sont les élèves à risque élevé qui profitent le plus des programmes de mise à niveau en littératie comme celui que nous proposons dans notre recherche-action-formation (Maltais, 2007; Dion, Brodeur, Gosselin, Campeau et Fuchs, 2010).

\section{$S A E$}

La SAÉ est un outil de planification pédagogique proposé entre autres par le MÉLS dans son renouveau pédagogique, ce qui était un gabarit assez nouveau pour les enseignantes de notre projet. La SAÉ est constituée d'activités d'évaluation des apprentissages en cours de route et de validation des acquis, tout cela organisé dans une tâche complexe (MÉLS, 2007; Gauthier, 2010; Riente, 2010). Elle comprend trois phases: préparation, réalisation et intégration, dans lesquelles sont structurées les activités d'apprentissage et d'évaluation. La phase de préparation présente la mise en situation de la SAÉ, permet aux élèves d'activer leurs connaissances antérieures quant au thème et aux connaissances visées, propose les objectifs du projet, précise la production attendue et donne les consignes de réalisation. La phase de réalisation est centrée davantage sur les activités d'apprentissage à réaliser selon les objectifs de la SAÉ et sur l'accompagnement que l'enseignante fera auprès de ses élèves. Elle prévoit des moments de rétroaction et d'objectivation après chacune des activités d'apprentissage ainsi qu'une ou des évaluations en cours d'apprentissage. La phase d'intégration prévoit des activités qui permettront aux élèves de faire une synthèse de leurs apprentissages et de transférer leurs connaissances et les compétences développées, et propose une évaluation finale des apprentissages. C'est aussi le moment de revenir sur la satisfaction des élèves quant au projet réalisé.

Dans notre recherche, nous nous sommes inspirées du gabarit créé par Gauthier (2010) afin de proposer aux enseignantes un canevas respectant les exigences de notre étude et les liens concrets que les enseignantes peuvent faire avec la littératie. Le gabarit de la SAÉ, ainsi utilisé par les enseignantes, est alors devenu à la fois un outil de planification et de différenciation pédagogique ainsi qu'un outil méthodologique de collecte de données.

\section{Besoins du milieu d'accueil sur le plan de la formation continue}

Sur le plan administratif, le directeur des trois petites écoles situées en milieu défavorisé, a tenu à répondre adéquatement aux besoins de ses enseignantes du préscolaire et du $1^{\text {er }}$ cycle du primaire. Déjà, celles-ci désiraient s'engager dans une démarche d'accompagnement rigoureuse afin d'améliorer leurs pratiques et elles voulaient être mises au courant des approches pédagogiques validées et reconnues, ce qui rejoint les propos de Paillé (1994), Lafortune (2006) ainsi que Prud'homme et coll. (2011) en termes de réflexivité sur leurs pratiques. Ensuite, elles souhaitaient briser leur isolement professionnel puisqu'elles sont seules à enseigner à leur niveau dans leur établissement. Le directeur nourrissait également le désir de consolider un travail de pluriprofessionnalité entre les enseignantes déjà entamé dans ces trois écoles, c'est-à-dire une concertation entre les divers intervenants gravi- 
tant autour des élèves : enseignantes, orthopédagogues des classes spéciales et orthopédagogues. Finalement, la CSRDN et les écoles ciblées, dans leurs plans de réussite ${ }^{7}$ en matière d'intervention précoce, tenaient compte de la Stratégie nationale d'alphabétisation précoce du Réseau canadien de recherche sur le langage et l'alphabétisation dans ses plans de réussite, notamment la recommandation 2 au sujet des stratégies d'enseignement rigoureuses et la recommandation 4 qui soutient l'échange de connaissances et de ressources en littératie (Réseau canadien de recherche sur le langage et l'alphabétisation, 2009, p. 10 et 12).

En somme, tous ces éléments nous ont amenées à proposer une démarche scientifique issue de la recherche-action (Paillé 1994; Charlier, 2005; Lafortune, 2006; Prud'homme et coll., 2011) qui nous permettrait à la fois de faire avancer les connaissances au sujet des pratiques enseignantes effectives en littératie au préscolaire et au $1^{\text {er }}$ cycle du primaire et d'accompagner un groupe d'enseignantes de manière prolongée en leur offrant une formation continue. Cette formation leur a permis de créer des SAÉ respectant un cadre théorique et les besoins de leurs élèves en littératie.

\section{Considérations théoriques}

La recherche-action-formation, encore peu documentée sur le plan méthodologique à l'heure actuelle, est un courant de recherche issu du paradigme qualitatif. Trois appellations sensiblement synonymes semblent acceptées : recherche-action-formation (Paillé, 1994; Charlier, 2005; Prud'homme et coll., 2011), recherche-formation (Marcel, 1999; Veyrunes, Bertone et Durand, 2003; Lafortune, 2006; Manço et Vaes-Harou, 2009) et recherche-action-intervention (Paillé, 1994; Lafortune, 2006). Nous retenons toutefois l'appellation recherche-action-formation qui nous semble plus représentative de ce que nous avons vécu dans notre projet et qui semble davantage calquée sur la réalité de qu'il se passe sur le terrain, ce que nous expliquons dans les paragraphes suivants.

\section{Recherche-action-formation}

La recherche-action-formation s'inscrit dans la foulée de la recherche-action, reconnue à part entière pour générer des connaissances (Prud'homme et Guay, 2011, p. 186). La recherche-action vise l'avancement des connaissances au sujet d'une situation pédagogique, et ce, par l'entremise de rencontres de praticiens-chercheurs qui permettent d'exprimer leurs différentes idées. «Elle repose sur l'idée que la recherche a avantage à s'orienter vers l'action, à s'ancrer dans l'expérience et à s'inscrire dans une perspective participative où praticiens et chercheurs collaborent à la solution de problèmes liés au développement de la pratique » (Prud’homme et Guay, 2011, p. 190-191). La recherche-action propose une méthodologie rigoureuse et souple à la fois, car elle préconise une démarche itérative favorisant un va-et-vient continu entre sa triple finalité, soit la recherche, l'action

7. École Saint-Joseph, CSRDN. Plan de la réussite et convention de gestion et de réussite éducative année scolaire. CSRDN, St-Jérôme, 2011-2012. École de la Durantaye, CSRDN. Plan de la réussite et convention de gestion et de réussite éducative année scolaire, CSRDN, Saint-Jérôme, 2011-2012. 
et la formation. Le volet recherche vise à améliorer l'état des connaissances sur la situation pédagogique et fait référence à une démarche scientifique traditionnelle qui s'interroge sur une réalité. Le volet action est l'élément central, car il est le trait d'union entre la recherche et la formation. L'action provoque un changement aux composantes de la situation pédagogique et est témoin d'une démarche commune entre le chercheur et l'acteur, soit ici les enseignantes des écoles ciblées. Le volet formation permet le développement professionnel des personnes qui vivent la situation pédagogique. La formation, dans notre cas la formation continue des enseignantes, est le lieu d'une réflexion intensive sur la pratique et sur ce qui y pose problème (Paillé, 1994; Dolbec et Clément, 2000; Karsenti et Savoie-Zajc, 2004; Prud'homme et Guay, 2011). En effet, c'est par la formation que les participants réfléchissent à leurs pratiques, assimilent et expérimentent de nouveaux concepts dans leur classe pour finalement y apporter des changements durables et une distanciation (Marcel, 1999, Lafortune, 2006). Charlier (2005) propose aussi quatre étapes pour réaliser une recherche-action-formation : l'action qui correspond à la formation des enseignants; la construction de la problématique, construction commençant avant l'action et se terminant après; l'élaboration d'un cadre conceptuel et du désign méthodologique ayant surtout lieu après la formation; la communication sous forme d'écriture (comptes rendus de recherche, documents de travail, journal de bord, articles, etc.) pour rejoindre la pratique, qui a lieu tout au long de la recherche.

De manière plus spécifique, la recherche-action-formation est à visée socioconstructiviste et s'adresseà un groupe restreint de participants (Paillé,1994; Marcel, 1999; Lafortune, 2006; Prud'homme et coll., 2011) - ou de formés selon Marcel (1999) - et s’appuie sur les savoirs, les théorisations des praticiens, redonnant ainsi toute sa légitimité à la « connaissance ordinaire » (Marcel, 1999, p. 97). Cela permet ensuite de générer une connaissance savante de ces pratiques, connaissance qui sera mise en mots par le chercheur (Paillé, 1994; Marcel, 1999). En effet, dans notre recherche, les enseignantes ont coconstruit leurs connaissances sur la littératie grâce aux séminaires et ont aussi coconstruit leurs SAÉ en équipes de travail en étant accompagnées de l'équipe de recherche. Également, ce type de recherche est une réponse à une demande sociale venant directement du milieu (Marcel, 1999; Lafortune, 2006; Prud'homme et coll., 2011) « [...] qui suppose des réflexions-interactions qui confrontent les pratiques, les croyances (conceptions et convictions) et suscite des conflits sociocognitifs avec l'objectif d'avoir plus de cohérence ou d'avoir conscience de ses incohérences, de les verbaliser, de les partager, de les discuter » (Lafortune, 2006). Ici, « [...] la recherche et la formation seraient en interrelation, conservant chacune leur spécificité tout en s'enrichissant réciproquement » (Marcel, 1999, p. 91). Finalement, la formation doit être assez longue afin de permettre aux participants de développer des compétences professionnelles clés, d'opérer des changements dans leurs pratiques et d'avoir des retombées concrètes pour les participants, par exemple la création de matériel didactique par les formés, les commentaires du chercheur par rapport à ce matériel, le réinvestissement des nouveaux savoir dans l'accompagnement de jeunes enseignants, la rédaction d'articles ou de livres, la présentation du projet dans des congrès ou colloques (Paillé, 1994; Marcel, 1999; Charlier, 2005; Lafortune, 2006; Prud'homme et coll., 2011). Afin de légitimer cette production didactique au sein du groupe de formés, il est nécessaire d'expérimenter ledit travail en classe, car 
les résultats de cette expérimentation sont également des données empiriques sur le changement des pratiques souhaité par la recherche-action-formation et permettent la régulation des pratiques des formés (Paillé, 1994).

En outre, l'ajout du mot formation dans la recherche-action-formation n'est pas anodin, loin de là. En effet, ce type de recherche allie « recherche de terrain à visée académique, réflexion sur la pratique, innovation pédagogique et formation diplômée [...] 》 (Paillé, 1994, p. 215). Certains chercheurs proposent toutefois l'idée que la formation ne soit pas nécessairement une formation diplômée, mais une formation continue (Marcel, 1999; Charlier, 2005; Lafortune, 2006). Le réinvestissement des apports de la formation est tributaire du désir du participant de changer ses propres pratiques : le chercheur n'y est pas associé (Marcel, 1999). Marcel (1999) suggère qu'un minimum de deux chercheurs, ou cochercheurs, travaillent de concert dans le projet, chacun répondant à une fonction précise qui peut être interchangeable selon les rencontres de travail, soit la direction de la recherche et la conduite du groupe de formés et le rôle de personne-ressource responsable de l'évaluation et de la régulation du fonctionnement du groupe. Ces deux rôles sont complémentaires, celui de personne-ressource exerçant une vigilance qui permet au chercheur principal de se concentrer sur sa posture scientifique. De plus, la formation proprement dite se doit de suivre des étapes bien précises et d'emprunter les méthodologies de la recherche en cours dans son déroulement afin d'être rigoureuse et de répondre aux besoins des formés. La partie formation devient, pour les participants, une formation à la recherche et les engage ainsi dans un processus de réflexion sur leur pratique qui demeurera ancré dans leurs schèmes pédagogiques (Paillé, 1994).

\section{Rigueur méthodologique de la recherche-action-formation}

Un reproche fait à l'égard de la recherche-action par certains tenants du positivisme et, par le fait même, de la recherche-action-formation, serait son supposé manque de rigueur méthodologique. Toutefois, plusieurs chercheurs, dont ceux cités dans notre article, ont démontré en quoi les outils de collecte de données de la recherche-action-formation pouvaient être rigoureux par leur complexité et leur quantité. N'oublions pas en effet que la recherche-action-formation fait état de pratiques en changement; documenter celles-ci demande nécessairement au chercheur de préparer un désign méthodologique souple, mais rigoureux, afin de pouvoir garder toutes les traces desdits changements, par exemple des comptes rendus in extenso, des comptes rendus de recherche, un journal de bord des participants et du chercheur, des entrevues semi-dirigées, des entretiens de groupe, des questionnaires d'enquête, des grilles d'observation participante ou non, etc. Ces outils permettent deux formes de triangulation nécessaires afin de garantir la rigueur de la recherche, soit la triangulation des méthodes - questionnaires, journaux de bord, entrevues, observation non participante, etc. - et la triangulation indéfinie - par la présentation de différents niveaux d'analyse en cours de recherche afin que les participants en fassent l'appréciation, par exemple leur validation des comptes rendus in extenso et du contenu des entrevues semi-dirigées (Dolbec et Clément, 2000; Prud'homme et Guay, 2011). Dans le même ordre d'idées, Paillé (1994) précise trois critères à respecter dans une 
recherche-action-formation : 1) méthodologie pour l'éducation : ce en quoi la recherche-action a fait ses preuves depuis deux décennies; 2) méthodologie pour l'innovation, soit permettre et réguler l'innovation pédagogique; 3) méthodologie pour la science, c'est-à-dire pour permettre l'avancement des connaissances.

\section{Objectifs}

Afin de tenir compte des préoccupations pédagogiques du directeur des trois écoles ciblées de répondre aux besoins des enseignants et de les amener à renouveler leurs pratiques en littératie tel qu'expliqué dans la problématique, la recherche-action-formation s'est avérée le meilleur choix. En effet, « Dans le cadre de la mise en œuvre d'un changement en éducation, nous ne pouvons concevoir un projet de recherche sans lui allier une formation associant théorie et pratique surtout dans le cas où le milieu scolaire est grandement associé à la démarche de recherche » (Lafortune, 2006, p. 193). Notre désign méthodologique, présenté en section 3, nous a permis d'atteindre nos objectifs, soit deux objectifs de formation et un objectif spécifique de recherche. Les objectifs de formation étaient les suivants : mettre en place une activité de formation en littératie (lecture et oral) sous forme de séminaire avec des enseignantes du préscolaire et du $1^{\text {er }}$ cycle du primaire et des orthopédagogues, et les accompagner dans la conception et l'expérimentation de SAÉ en littératie. L'objectif spécifique de recherche était d'étudier l'impact de ce séminaire sur l'appropriation du concept de littératie et sur le renouvèlement de leurs pratiques.

\section{Désign méthodologique}

Dans cette section, nous présentons les trois volets de notre désign méthodologique : recherche, action et formation. Nous poursuivons avec les avantages et les limites de la recherche-action-formation.

Nous rappelons que notre recherche-action-formation a été subventionnée par le Programme de formation continue du personnel scolaire du MÉLS de 2010 à 2012. Notre désign méthodologique allie la recherche au regard du renouvèlement des pratiques des enseignantes en littératie volets lecture (an 1) et oral (an 2), l'action dans la conception et l'expérimentation de SAÉ inédites en lecture et en oral intégrant la littératie et la formation à la littératie, concept nouveau pour les participantes, et à la SAÉ, outil de planification pédagogique méconnu des enseignantes. Nous rappelons aussi que notre équipe de recherche-action-formation est constituée de la professeure-chercheuse et d'un cochercheur principal, le directeur des trois écoles, et d'autres cochercheuses travaillant à la CSRDN : l'orthopédagogue d'une des écoles ciblées et trois conseillères pédagogiques. Ces six personnes constituaient l'équipe conceptrice et étaient présentes à chaque étape de la recherche-action-formation. À ces cochercheurs se sont ajoutés trois professeurs-chercheurs qui sont intervenus de manière ponctuelle dans les séminaires de formation en donnant chacun une conférence sur un sujet particulier. 
Nos activités de formation se déroulaient sous la forme de séminaires de deux jours chacun, ce qui constitue un procédé pédagogique qui s'adresse à un groupe restreint et dont le but est l'exploration collective d'un objet d'étude (Legendre, 2005), ici la littératie pour les volets lecture et oral, et au cours duquel les participants étudient et débattent ensemble du contenu préalablement défini. Une large part d'initiative leur est laissée puisque les échanges entre les participants occupent de 70 à $75 \%$ du séminaire et jouent un rôle-clé. En effet, l'exploration du sujet se fait par leur intermédiaire. Entre chacun des séminaires, les enseignantes avaient une journée de libération pour travailler à l'élaboration de leur SAÉ. Notre désign méthodologique s'appuie sur celui d'une recherche antérieure dans laquelle un programme de formation a mobilisé 18 enseignants néobrunswickois de français du secondaire (Lafontaine, Dezutter et Thomas, 2010). À des moments-clés de la recherche, nous avons recueilli des données afin de documenter le renouvèlement des pratiques des enseignantes. Le tableau 1 présente une vue d'ensemble de notre recherche-action-formation, qui sera expliquée dans les sections suivantes. 
Tableau 1 : Vue d'ensemble de la recherche-action-formation

\begin{tabular}{|c|c|c|c|c|}
\hline \multicolumn{5}{|c|}{ An 1 2010-2011 volet LECTURE } \\
\hline Juin & Sept. & Oct. & Nov. & Déc. \\
\hline $\begin{array}{l}\text { Rencontre préparatoire } \\
\text { équipe conceptrice } \\
\text { Rencontre enseignantes } \\
\text { pour cerner besoins et } \\
\text { perceptions lecture-littéra- } \\
\text { tie-SAÉ }\end{array}$ & $\begin{array}{l}\text { Rencontre préparatoire } \\
\text { équipe } \\
\text { Séminaire } 1 \text { incluant travail } \\
\text { d'équipe SAÉ phase de } \\
\text { préparation } \\
\text { Questionnaire 1-lecture }\end{array}$ & $\begin{array}{l}\text { Libération } 1 \\
\text { Rencontre préparatoire } \\
\text { équipe conceptrice }\end{array}$ & $\begin{array}{l}\text { Séminaire } 2 \text { incluant travail } \\
\text { d'équipe SAÉ phase de } \\
\underline{\text { réalisation }} \\
\text { Entretien de groupe } 1\end{array}$ & $\begin{array}{l}\text { Libération } 2 \\
\text { Rencontre préparatoire } \\
\text { équipe conceptrice }\end{array}$ \\
\hline \multicolumn{5}{|c|}{ An 2 2011-2012 volet ORAL } \\
\hline $\begin{array}{l}\text { Rencontre préparatoire } \\
\text { équipe conceptrice }\end{array}$ & $\begin{array}{l}\text { Séminaire } 5 \text { incluant travail } \\
\text { d'équipe SAÉ phase de } \\
\text { préparation } \\
\text { Questionnaire 1-oral }\end{array}$ & $\begin{array}{l}\text { Libération } 5 \\
\text { Diffusion: communica- } \\
\text { tion (directions d'écoles } \\
\underline{\text { CSRDN) }}\end{array}$ & $\begin{array}{l}\text { Séminaire } 6 \underline{\text { incluant travail }} \\
\frac{\text { d'équipe SAÉ phase de }}{\text { réalisation }} \\
\text { Entretien de groupe } 3\end{array}$ & $\begin{array}{l}\text { Libération } 6 \\
\text { Diffusion: communication } \\
\underline{\text { (AQEP) }}\end{array}$ \\
\hline
\end{tabular}

\begin{tabular}{|c|c|c|c|c|}
\hline \multicolumn{5}{|c|}{ An 1 2010-2011 volet LECTURE (suite) } \\
\hline Janvier & Février & Mars & Avril & Mai, juin, juillet \\
\hline $\begin{array}{l}\text { Séminaire } 3 \text { incluant } \\
\text { travail d'équipe SAÉ phase } \\
\underline{\text { d'intégration }}\end{array}$ & Libérations 3-4 & $\begin{array}{l}\text { Expérimentation en classe } \\
\text { des SAÉ } \\
\text { Rencontre préparatoire } \\
\text { équipe conceptrice }\end{array}$ & $\begin{array}{l}\text { Séminaire } 4 \\
\text { Questionnaire 2-lecture } \\
\text { Entretien de groupe } 2 \\
\text { Entrevues } 1\end{array}$ & $\begin{array}{l}\text { Rencontre de bilan équipe } \\
\text { conceptrice } \\
\text { Diffusion : articles et } \\
\text { communications (ACFAS } \\
\text { et Conférence européenne } \\
\underline{\text { sur la lecture) }}\end{array}$ \\
\hline \multicolumn{5}{|c|}{ An 2 2011-2012 volet ORAL (suite) } \\
\hline $\begin{array}{l}\text { Séminaire } 7 \underline{\text { incluant }} \\
\text { travail d'équipe SAÉ phase } \\
\underline{\text { d'intégration }}\end{array}$ & Libérations 7-8 & Expérimentation SAÉ & Expérimentation SAÉ & $\begin{array}{l}\text { Séminaire } 8 \\
\text { Questionnaire2-oral } \\
\text { Entretien de groupe } 4 \\
\text { Entrevues } 2 \\
\text { Rencontre de bilan } \\
\text { Rencontre enseignantes } \\
\text { pour cerner besoins et per- } \\
\text { ceptions oral-littératie-SAÉ } \\
\text { Diffusion: articles, col- } \\
\text { loques, etc. }\end{array}$ \\
\hline
\end{tabular}

Légende : caractères italique = recherche; caractères soulignés = action; caractères réguliers = formation

\section{Recherche-action-formation : volet recherche}

L'objectif spécifique de recherche était d'étudier l'impact de ce séminaire sur l'appropriation du concept de littératie et sur le renouvèlement des pratiques. Nous nous intéressions aux perceptions des enseignantes au regard de la littératie et de leur évolution au fil du projet ainsi qu'au renouvèlement de leurs pratiques à la suite de la conception et de l'expérimentation de leur SAÉ.

Dès l'obtention de la subvention en avril 2010, nous avons tenu une première rencontre avec notre équipe conceptrice afin de partager notre vision de cette recherche-action-formation, de définir les 
rôles de chacun, d'attribuer les tâches à chacun et de préparer la première rencontre avec les enseignantes. Nous nommons cette réunion « rencontre de départ » qui se situe avant l'action. Le directeur nous a également donné le portrait des participantes: deux enseignantes du préscolaire, neuf du $1^{\text {er }}$ cycle du primaire (deux en adaptation scolaire, trois en classe langage et quatre au régulier; six au $1^{\text {er }}$ cycle et trois au $2^{\mathrm{e}}$ cycle) et les deux orthopédagogues-école. Notons que toutes les enseignantes du $1^{\text {er }}$ cycle des trois écoles ciblées étaient impliquées dans le projet ainsi que le directeur adjoint d'une des écoles ciblées 8 .

Par la suite, en juin 2010, l'équipe conceptrice a rencontré les enseignantes afin de leur expliquer le projet et de recueillir leurs besoins en lecture et leurs premières perceptions de la littératie. Nous avons posé cinq questions aux enseignantes en lien avec les objectifs du projet qui étaient affichés sur cinq grandes feuilles. En équipes de quatre personnes, les enseignantes échangeaient et circulaient pour répondre à chacune des questions et ainsi créer un graffiti collectif de leurs représentations et de leurs besoins. L'encadré 1 présente les questions posées.

\section{Encadré 1 : Questions préparatoires au séminaire 1}

1. Comment définiriez-vous vos pratiques en littératie?

Ce que je fais...

Ce dontj'ai besoin...

2. Quels obstacles potentiels voyez-vous au développement de la littératie dans votre milieu?

Ce que je sais...

Ce dontj'ai besoin...

3. Comment pourrait-on possiblement exploiter la littératie chez les élèves?

Ce que je sais...

Ce dontj'ai besoin...

4. Quel pourrait être l'apport positif des parents dans le développement la compétence à lire de vos élèves?

Ce que je sais...

Cedontj'ai besoin...

5. Qu'évoque pour vous le concept de situation d'apprentissage et d'évaluation (SAÉ)? Quelle représentation vous en faites-vous?

Ce que je sais...

Ce dontj'ai besoin...

8. Raymond Rochette est le directeur adjoint de l'école Saint-Joseph. 
Nous avons fait une synthèse des réponses afin de nous rencontrer de nouveau pour préparer le premier séminaire. Ces réponses nous ont été fort utiles afin de cibler ce qu'elles savaient, ce dont elles avaient besoin sur le plan de la formation continue et leurs premières perceptions de la littératie volet lecture ainsi que de l'outil de planification pédagogique, qu'est la SAÉ, utilisé dans le projet.

Tel que décrit dans le tableau 1, nous avons fait une rencontre de préparation en équipe conceptrice avant chacun des séminaires. Chaque rencontre suivait un ordre du jour selon un canevas proposé par Paillé (1994) permettant de répondre aux trois finalités de la recherche-action-formation : un point recherche pour préparer ou revenir sur la collecte des données, un point action pour prévoir le travail d'équipe des enseignantes de la conception de leur SAÉ, préparer et discuter de l'expérimentation, et un point formation pour organiser ladite formation, son contenu, les ajustements à y faire et la gestion du budget. Un point divers était également prévu. Notre professionnelle de recherche assistait à chaque rencontre afin de rédiger un compte rendu qui consignait des traces de notre projet, données documentaires d'une importance capitale sur les plans méthodologique et organisationnel (Paillé, 1994; Prud'homme et coll., 2011). L'encadré 2 donne un exemple d'ordre du jour des rencontres préparatoires de l'équipe conceptrice. 


\section{Encadré 2 : Exemple d'ordre du jour d'une rencontre préparatoire}

\section{Rencontre préparatoire du 13 octobre 2010}

\section{Point formation}

1. Retour sur le séminaire 2 : réactions, suivi, commentaires des enseignantes

2. Séminaire 3 : contenu et organisation

3. Organisation du séminaire 4 : conférencier proposé XXXX, préparation, matériel, demandes aux enseignantes, etc.

4. Achat de matériel didactique : suivi

\section{Point action}

5. Séminaire 3 : retour sur les SAÉ, travail sur la phase d'intégration et présentation des centres de littératie, tâche de libération 3-4

\section{Point recherche}

6. Retour sur le compte-rendu de la rencontre du XXXX

7. Validation des SAÉ : rôles de chacun, échéancier

8. Collecte des données : transcription des questionnaires 1 et des entretiens de groupe 1

9. Analyse des données : analyse de contenu avec le logiciel N'Vivo

10. Suivi symposium Belgique et congrès AQEP décembre

Divers

Consignation des données dans Dropbox

Prochaine rencontre

Tout au long de la recherche, comme l'indique le tableau 1, nous avons recueilli des données sur l'appropriation du concept de littératie et de l'outil pédagogique qu'est la SAÉ ainsi que sur le renouvèlement des pratiques des enseignantes à l'aide de divers instruments de collecte de données que voici expliqués et illustrés:

- questionnaire écrit sur les perceptions de la littératie volets lecture (septembre 2010) et oral (septembre 2011). Répondu deux fois à des moments clés de la recherche, ce questionnaire nous a permis de voir l'évolution des enseignantes quant à leur pensée théorique et à leurs pratiques pédagogiques et de les comparer (voir tableau 2); 


\section{Tableau 2 : Questionnaires écrits sur les perceptions des enseignantes}

\begin{tabular}{|c|c|}
\hline Volet LECTURE septembre 2010 & Volet ORAL septembre 2011 \\
\hline $\begin{array}{l}\text { - Selon vous, qu'est-ce que la littératie? } \\
\text { - Selon vous, comment la lecture est-elle intégrée au concept de } \\
\text { littératie? } \\
\text { - Selon vous, comment peut-on travailler la littératie volet lecture en } \\
\text { classe? } \\
\text { - Selon vous, qu'est-ce qu'une situation d'apprentissage et d'évalua- } \\
\text { tion (SAÉ)? }\end{array}$ & $\begin{array}{l}\text { - Selon vous, qu'est-ce que la littératie? } \\
\text { - Selon vous, comment l'oral est-il intégré au concept de littératie? } \\
\text { - Selon vous, comment peut-on travailler la littératie volet oral en } \\
\text { classe? } \\
\text { - Selon vous, qu'est-ce qu'une situation d'apprentissage et d'évalua- } \\
\text { tion (SAÉ) en oral? }\end{array}$ \\
\hline
\end{tabular}

- entretiens de groupe filmés et réalisés aux rencontres 2, 4, 6 et 8. Ceux-ci nous ont permis a) de vérifier la compréhension par les participants du concept de littératie volets lecture et oral et de l'outil pédagogique qu'est la SAÉ; b) de recueillir de nouvelles informations à propos des implications pédagogiques du travail par SAÉ en littératie au départ de l'observation critique d'exemples de pratiques enregistrées au cours des périodes d'expérimentation (voir tableau 3). Chaque participante s'était vu attribuer un numéro afin de garantir son anonymat;

Tableau 3 : Canevas entretien de groupe

\begin{tabular}{|c|c|}
\hline Volet LECTURE avril 2011 & Volet ORAL mai 2012 \\
\hline $\begin{array}{l}\text { 1. Depuis le séminaire } 1 \text {, quelle est votre conception du concept de } \\
\text { littératie? }\end{array}$ & $\begin{array}{l}\text { 1. Depuis le séminaire } 5 \text { (début du volet oral), quelle est votre conception } \\
\text { de la littératie? }\end{array}$ \\
\hline $\begin{array}{l}\text { 2. Depuis le séminaire } 1 \text {, pouvez-vous expliquer comment la lecture } \\
\text { peut être intégrée au concept de littératie? }\end{array}$ & $\begin{array}{l}\text { 2. Depuis le séminaire 5, pouvez-vous expliquer comment l'oral peut être } \\
\text { intégré au concept de littératie? }\end{array}$ \\
\hline $\begin{array}{l}\text { 3. Depuis le séminaire } 1 \text {, avez-vous des pistes pour travailler la } \\
\text { littératie volet lecture en classe? Lesquelles? }\end{array}$ & $\begin{array}{l}\text { 3. Depuis le séminaire 5, avez-vous des pistes pour travailler la littératie } \\
\text { volet oral en classe? Lesquelles? }\end{array}$ \\
\hline $\begin{array}{l}\text { 4. Depuis le séminaire } 1 \text {, quelle est votre conception du concept de } \\
\text { situation d'apprentissage et d'évaluation (SAÉ)? }\end{array}$ & $\begin{array}{l}\text { 4. Depuis le séminaire } 5 \text {, quelle est votre conception du concept de situa- } \\
\text { tion d'apprentissage et d'évaluation (SAÉ) volet oral? }\end{array}$ \\
\hline $\begin{array}{l}\text { 5. Le fonctionnement et le contenu du séminaire vous amènent- } \\
\text { ils à mieux comprendre les concepts de littératie et de SAÉ? } \\
\text { Comment? }\end{array}$ & $\begin{array}{l}\text { 5. Avez-vous modifié vos pratiques d'enseignement en lecture et en oral } \\
\text { depuis le séminaire 1? Comment? }\end{array}$ \\
\hline
\end{tabular}

- journaux de bord individuel et d'équipe de la SAÉ qui ont permis de garder des traces de l'expérimentation de la SAÉ. Le journal de bord individuel servait à garder les observations à chaud de chacune des enseignantes pendant l'expérimentation (voir encadré 3). Le journal de bord d'équipe était rempli au dernier séminaire de chaque année (séminaires 4 et 8) afin que les enseignantes de chacune des équipes mettent en commun leurs observations et se mettent à distance par rapport à l'expérimentation pour ainsi pouvoir mieux réfléchir au renouvèlement de leurs pratiques et en discuter en entrevue semi-dirigée individuelle (voir encadré 4); 
Encadré 3 : Journal de bord individuel

\begin{tabular}{|l|l|}
\hline Phase: & $\square$ Préparation $\square$ Réalisation $\square$ Intégration \\
\hline Titre de l'activité : & $\begin{array}{l}\text { Réactions des élèves face à la tâche et au fonctionnement (affectives, cognitives, relationnelles, comportementales) } \\
\text { Bons coups, difficultés, à refaire, autres }\end{array}$ \\
\hline$* * *$ À répéter pour chaque activité et pour chacune des phases de la SAÉ. \\
\hline
\end{tabular}




\section{Encadré 4 : Journal de bord d'équipe}

\section{Phase de préparation : la mise en situation}

Quoi? Pourquoi? Quand?

Le rappel des connaissances antérieures (déclaratives, conditionnelles, procédurales)

- Activer les connaissances antérieures des élèves en lien avec la thématique de la SAÉ et leur intention de lecture.

À la lumière de mes consignations en lien avec la phase de préparation, je fais une synthèse de mes observations:

Ce que j'ai appris sur ma pratique professionnelle ou mon milieu

Ce que je ferais différemment la prochaine fois, s'il y a lieu

Phase de réalisation : l'expérience d'apprentissage

Comment?

Le déroulement

À la lumière de mes consignations en lien avec la phase de réalisation, je fais une synthèse de mes observations:

Ce que j'ai appris sur ma pratique professionnelle ou mon milieu

Ce que je ferais différemment la prochaine fois, s'il y a lieu

Phase d'intégration : le transfert

Quand?

Le déroulement

À la lumière de mes consignations en lien avec la phase d'intégration, je fais une synthèse de mes observations:

Ce que j'ai appris sur ma pratique professionnelle ou mon milieu

Ce que je ferais différemment la prochaine fois, s'il y a lieu 
- $\quad$ entrevue semi-dirigée individuelle enregistrée et réalisée aux rencontres 4 et 8, donc à la fin de chaque année du projet, afin de comprendre l'impact du dispositif de formation sur l'appropriation du concept de littératie et de la SAÉ ainsi que sur le renouvèlement de leurs pratiques pédagogiques (voir canevas tableau 4). Chaque participante avait le même numéro que lors de l'entretien de groupe afin de garantir son anonymat.

\section{Tableau 4 : Canevas des entrevues semi-dirigées}

\begin{tabular}{|c|c|}
\hline $\begin{array}{c}\text { Entrevue } 1 \text { volet LECTURE } \\
\text { Avril } 2011\end{array}$ & $\begin{array}{c}\text { Entrevue } 2 \text { volet ORAL } \\
\text { Mai } 2012\end{array}$ \\
\hline $\begin{array}{l}\text { Bloc } 1 \text { : Appropriation du concept de littératie volet LECTURE } \\
\text { 1. Quelle est votre conception de la littératie volet lecture? } \\
\text { 2. Vous sentez-vous à l'aise maintenant avec ce concept? Expliquez. } \\
\text { 3. Comment le séminaire de formation vous a-t-il permis de mieux } \\
\text { comprendre le concept de littératie? }\end{array}$ & $\begin{array}{l}\text { Bloc } 1 \text { : Appropriation du concept de littératie volet ORAL } \\
\text { 1. Quelle est votre conception de la littératie volet oral? } \\
\text { 2. Vous sentez-vous à l'aise maintenant avec ce concept? Expliquez. } \\
\text { 3. Comment le séminaire de formation vous a-t-il permis de mieux } \\
\text { comprendre le concept de littératie? }\end{array}$ \\
\hline $\begin{array}{l}\text { Bloc } 2 \text { : Appropriation du concept de la SAÉ } \\
\text { 1. Quelle est votre conception de la SAÉ? } \\
\text { 2. Pouvez-vous nous parler des effets généraux que la SAÉ en lecture a } \\
\text { sur l'enseignement et l'apprentissage du français? } \\
\text { 3. Pouvez-vous nous parler des effets que l'outil pédagogique de la SAÉ } \\
\text { a eus sur votre façon d'enseigner? } \\
\text { 4. Vous sentez-vous à l'aise maintenant avec cet outil? } \\
\text { 5. Comment le séminaire de formation vous a-t-il permis de mieux } \\
\text { comprendre cet outil? }\end{array}$ & $\begin{array}{l}\text { Bloc } 2 \text { : Appropriation du concept de la SAÉ } \\
\text { 1. Quelle est votre conception de la SAÉ en oral? } \\
\text { 2. Pouvez-vous nous parler des effets généraux que la SAÉ en oral a sur } \\
\text { l'enseignement et l'apprentissage du français? } \\
\text { 3. Pouvez-vous nous parler des effets que l'outil pédagogique de la SAÉ } \\
\text { a eus sur votre façon d'enseigner? } \\
\text { 4. Vous sentez-vous à l'aise maintenant avec cet outil? } \\
\text { 5. Comment le séminaire de formation vous a-t-il permis de mieux } \\
\text { comprendre cet outil? }\end{array}$ \\
\hline $\begin{array}{l}\text { Bloc } 3 \text { : Renouvèlement des pratiques enseignantes en littératie } \\
\text { volet LECTURE } \\
\text { 1. Quelle sont les particularités de vos élèves en littératie volet lecture? } \\
\text { 2. Avez-vous observé des changements chez vos élèves depuis la mise } \\
\text { en pratique de votre SAÉ en littératie volet lecture? Lesquels? } \\
\text { 3. Qu'avez-vous changé dans vos pratiques enseignantes depuis votre } \\
\text { participation au séminaire? } \\
\text { 4. Ces changements sont-ils positifs ou négatifs? } \\
\text { Expliquez. } \\
\text { 5. Comment intégrez-vous maintenant la littératie volet lecture à vos } \\
\text { pratiques quotidiennes en classe? }\end{array}$ & $\begin{array}{l}\text { Bloc } 3 \text { : Renouvèlement des pratiques enseignantes en littératie } \\
\text { volet ORAL } \\
\text { 1. Est-ce que le séminaire volet oral vous a donné des pistes d'ensei- } \\
\text { gnement de l'oral? Lesquelles? Les avez-vous mises en pratique en } \\
\text { classe? Comment? } \\
\text { 2. Quelles sont les particularités de vos élèves en littératie volet oral? } \\
\text { 3. Avez-vous observé des changements chez vos élèves depuis la mise } \\
\text { en pratique de votre SAÉ en littératie volet oral? Lesquels? } \\
\text { 4. Qu'avez-vous changé dans vos pratiques enseignantes depuis votre } \\
\text { participation au séminaire? } \\
\text { 5. Ces changements sont-ils positifs ou négatifs? } \\
\text { Expliquez. } \\
\text { 6. Comment intégrez-vous maintenant la littératie volet oral à vos } \\
\text { pratiques quotidiennes en classe? } \\
\text { 7. Que ferez-vous l'an prochain dans vos classes en littératie lecture et } \\
\text { en littératie oral? } \\
\text { 8. Avez-vous quelque chose à ajouter? }\end{array}$ \\
\hline
\end{tabular}

À chacune des années de la recherche, les données ont été analysées à l'aide du logiciel d'analyse de données qualitative N’Vivo à partir de catégories déterminées par notre cadre conceptuel en littératie, en lecture et par notre outil pédagogique de la SAÉ. Lors du dernier séminaire de chaque année, nous avons fait un retour sur l'ensemble de la démarche avec les enseignantes et en équipe conceptrice.

9. Nous remercions Jessica Jordan et Julie Duchesne, assistantes de recherche, pour leur travail d'analyse des données. 


\section{Recherche-action-formation : volet action}

Ce volet action concerne spécifiquement la conception des SAÉ par les enseignantes, leur accompagnement à l'intérieur et à l'extérieur du séminaire par l'équipe conceptrice pendant cette élaboration, ainsi que son expérimentation en classe qui les a amenées à changer leurs pratiques. Pour travailler notre objectif de formation 2, à savoir accompagner les enseignantes dans la conception et l'expérimentation de SAÉ en littératie, les modalités de travail étaient les suivantes. Les enseignantes ont d'abord formé des équipes interécoles de trois ou quatre membres. Tel que montré dans le tableau 1, chaque séminaire comprenait une partie de travail d'équipe pour chacune des phases de la SAÉ. Les trois conseillères pédagogiques faisant partie de l'équipe conceptrice ont joué un rôle-clé dans cette partie des séminaires, car elles ont pu répondre à diverses questions des enseignantes pendant leur travail en séminaire. Entre chaque séminaire, les enseignantes étaient libérées une journée pour continuer à élaborer leur SAÉ. Au séminaire suivant, nous animions une discussion sur l'état d'avancement des SAÉ, répondions aux questions des enseignantes, etc., afin de leur assurer un accompagnement durant le séminaire. À chacune des étapes de la construction de la SAÉ (phase de préparation : septembre; phase de réalisation : octobre; phase d'intégration : janvier), les enseignantes nous remettaient leur travail que nous validions d'un point de vue théorique. Pour ce faire, nous avons utilisé la fonction commentaires du logiciel Word. Par la suite, la chercheuse principale rencontrait les équipes dans leur école afin de les leur expliquer. Ces rencontres avec les enseignantes dans leur école ont été extrêmement appréciées des participantes, car elles favorisaient un échange direct avec l'équipe conceptrice, ce qui permettait de clarifier les questions théoriques et de créer un conflit sociocognitif nécessaire au changement des pratiques (Lafortune, 2006). Selon Prud'homme et coll. (2011, p. 183), dans une recherche-action-formation, les participants ont grandement besoin de ce soutien individuel du chercheur ou de son équipe pour « [...] accroitre une activité réflexive qui permet de comprendre le sens de l'expérience de formation qu'elles vivent. » Les enseignantes profitaient ainsi d'une mise à distance essentielle afin de modifier ensuite leur SAÉ (Lafortune, 2006). Elles les ont finalement expérimentées en mars 2011 (SAÉ lecture) et en avril 2012 (SAÉ oral) en documentant leur démarche dans le journal de bord individuel. Au dernier séminaire de chaque année, chaque équipe enseignante a mis en commun ses observations et a complété le journal de bord d'équipe tel qu'expliqué à la section précédente.

Un autre élément très important du volet action se rapporte aux retombées directes du projet sur les enseignantes et leurs élèves, ce que nous avons fortement encouragé tout au long de la recherche. La création d'un matériel didactique inédit, les SAÉ en lecture et en oral intégrant la littératie (à notre connaissance, ce type de SAÉ n'existe pas), a lancé les enseignantes dans l'action; elles ont expérimenté les SAÉ et ont commenté leur impact sur leurs pratiques et sur leur appropriation du concept de littératie. Deux enseignantes ont également donné une communication avec la chercheuse principale et une conseillère pédagogique au congrès de l'Association québécoise des enseignantes et des enseignantes du primaire (AQEP); cinq enseignantes et une autre conseillère pédagogique ont publié avec nous leur SAÉ dans des revues professionnelles; trois autres enseignantes et deux 
conseillères pédagogiques ont travaillé avec la chercheuse principale à l'élaboration d'un feuillet d'information sur la littératie familiale à l'intention des parents de milieux défavorisés. Le volet action est également très important pour les membres de l'équipe conceptrice : les résultats du projet ont été présentés dans un congrès international et au congrès de l'Association francophone pour le savoir (ACFAS).

\section{Recherche-action-formation : volet formation}

Le volet formation permet le développement des personnes qui vivent la situation pédagogique et favorise une réflexion intensive à sa pratique et à ce qui y pose problème (Paillé, 1994; Dolbec et Clément, 2000; Karsenti et Savoie-Zajc, 2004; Prud'homme et Guay, 2011).

La formation a été le moment fort de notre projet, car elle nourrissait les enseignantes afin de concevoir leur SAÉ intégrant des moyens concrets en littératie. Il y avait toujours un va-et-vient entre la recherche - nous recueillions des données sur les perceptions et les pratiques des enseignantes - l'action - les participantes travaillaient en équipe pendant les séminaires avec notre soutien - et la formation concentrée sur la littératie volets lecture (an 1) et oral (an 2). Nos séminaires de formation étaient organisés ainsi : présentation théorique d'un concept ou conférence sur le concept, suivie d'une objectivation en grand groupe, activités de transfert des connaissances (modelage, pratique guidée, exercices, partage de matériel, etc.) suivies d'une objectivation en grand groupe, travail d'équipe pour la conception de la SAÉ avec accompagnement intégré. Les séminaires volet lecture ont abordé les concepts et notions suivantes: littératie volet lecture, fondements en lecture, acquisition chez les élèves de la conscience phonologique et phonémique, enseignement systématique et explicite de la lecture et des stratégies de lecture (décodage et entrée graphophonétique), développement du modèle à trois niveaux d'intervention et de l'interrelation entre chaque niveau (Haager, Klingner et Vaughn, 2007), développement du modèle d'intervention orthopédagogique et du pistage des progressions en lecture, outil de planification pédagogique SAÉ : contenu et gabarit à utiliser. Le contenu du séminaire volet oral a traité de la conscience phonologique, de l'enseignement explicite des stratégies de prise de parole et d'écoute, de l'oral pragmatique (Maurer, 2001; Dumais, 2011), démarche d'enseignement explicite de la production orale de Lafontaine (2011; Lafontaine et Préfontaine, 2007), du projet d'écoute et d'activités de compréhension orale. Une proportion de 70 à $75 \%$ du temps était consacrée aux échanges de diverses manières : objectivation suivant les présentations théoriques, discussions de groupe, partage d'expérience, travail d'équipe. Les tableaux 5 et 6 donnent un exemple de contenu d'un séminaire en lecture et d'un séminaire en oral. 
Tableau 5 : Exemple de contenu du séminaire en littératie, volet LECTURE

\begin{tabular}{|c|c|}
\hline $\begin{array}{c}\text { JOUR } 1 \\
12 \text { avril } 2011\end{array}$ & $\begin{array}{c}\text { JOUR } 2 \\
14 \text { avril } 2011\end{array}$ \\
\hline De 8 h 30 à 11 h 30 & De $8 \mathrm{~h} 30$ à $11 \mathrm{~h}$ \\
\hline $\begin{array}{l}\text { Retour en grand groupe sur l'expérimentation des SAÉ : points forts, } \\
\text { points à améliorer, anecdotes, etc. }\end{array}$ & $\begin{array}{l}\text { Suite du travail d'équipe, évaluation du séminaire et des entrevues } \\
\text { semi-dirigées }\end{array}$ \\
\hline Visionnage d'extraits & Visionnage de quelques extraits de SAÉ \\
\hline Partage coopératif & Objectivation de l'année de la part de l'équipe, participation aux \\
\hline Entretien de groupe 2 & congrès, diffusion SAE écrites et vidéo, présentation an 2, collecte des \\
\hline $\begin{array}{l}\text { Travail d'équipe : mise en commun par équipe des expérimentations, } \\
\text { journal de bord et version finale de la SAÉ }\end{array}$ & \\
\hline \multicolumn{2}{|l|}{ Entrevues semi-dirigées pendant le travail d'équipe } \\
\hline Diner : de $11 \mathrm{~h} 30$ à $13 \mathrm{~h}$ & Diner : de 11 h à 12 h 30 \\
\hline De $13 \mathrm{~h}$ à $15 \mathrm{~h} 30$ & De 12 h 30 à 15 h \\
\hline \multirow{4}{*}{$\begin{array}{l}\text { - Travail d'équipe : mise en commun par équipe des expérimentations, } \\
\text { journal de bord et version finale de la SAÉ } \\
\text { - Entrevues semi-dirigées pendant le travail d'équipe }\end{array}$} & Conférence de XXXX sur le logiciel Destination Littératie \\
\hline & Retour sur la conférence, évaluation du séminaire, mot de clôture \\
\hline & De 15 h 30 à 16 h 30 \\
\hline & Rencontre de diffusion congrès enseignantes intéressées \\
\hline
\end{tabular}

Tableau 6 : Exemple de contenu du séminaire en littératie, volet ORAL

\begin{tabular}{|c|c|}
\hline $\begin{array}{c}\text { JOUR } 1 \\
21 \text { septembre } 2011\end{array}$ & $\begin{array}{c}\text { JOUR } 2 \\
29 \text { septembre } 2011\end{array}$ \\
\hline De 8 h 30 à 11 h 30 & De 8 h 30 à 11 h 30 \\
\hline Accueil et anecdotes estivales en littératie & Retour sur le « devoir » : thématiques et moyens concrets en littératie \\
\hline $\begin{array}{l}\text { Retour sur le projet et les résultats de l'an } 1 \text { Questionnaire sur les per- } \\
\text { ceptions de la littératie volet oral }\end{array}$ & \multirow[t]{4}{*}{ Conférence de XXXX sur la conscience phonologique } \\
\hline Retour sur le graffiti collectif & \\
\hline Pause vers $10 \mathrm{~h}$ & \\
\hline Diaporama : la littératie volet oral et l'enseignement de l'oral, échanges & \\
\hline Diner de groupe $11 \mathrm{~h} 30$ à $13 \mathrm{~h}$ & Diner 11 h 30 à 13 h \\
\hline De 13 h à 15 h 30 & De $13 \mathrm{~h}$ à $15 \mathrm{~h} 30$ \\
\hline $\begin{array}{l}\text { Suite du diaporama : la littératie volet oral et l'enseignement de l'oral, } \\
\text { échange } \\
\text { Équipes de travail : remue-méninges thématique, moyens concrets en } \\
\text { littératie, phase de préparation } \\
\text { Présentation de références (bibliographie) } \\
\text { «Devoir » pour le } 29 \text { septembre } \\
\text { Visite à la didacthèque : matériel en oral }\end{array}$ & $\begin{array}{l}\text { Retour sur la conférence } \\
\text { Retour sur le gabarit de la SAÉ volet oral } \\
\text { Travail d'équipe : phase de préparation Tâche de libération pour le } 17 \\
\text { octobre }\end{array}$ \\
\hline
\end{tabular}

Pendant les séminaires, de nouveaux besoins de formation étaient identifiés par les enseignantes. Une conseillère pédagogique était responsable de les prendre en note afin que l'équipe conceptrice puisse y répondre au séminaire suivant. Par exemple, lors d'un séminaire sur l'oral, les enseignantes ont manifesté le besoin d'être outillées face à des difficultés bien particulières de leurs élèves en oral : faire parler les élèves silencieux, intervenir quand un enfant commet des fautes à l'oral, conserver des traces de l'oral. Forte de ces demandes, la chercheuse principale a pu y répondre au séminaire 
suivant en présentant des pistes théoriques et pratiques aux enseignantes. Après chacun des séminaires, l'équipe conceptrice faisait un retour afin de faire le point sur les faits saillants du séminaire, les points à améliorer et les pistes de réflexion pour la prochaine rencontre de préparation.

En somme, la formation donnée à chacun des séminaires était tout de suite réinvestie dans l'élaboration de la SAÉ et était remise sur la table lors de la validation des commentaires sur chaque étape de la SAÉ que la chercheuse donnait à chacune des équipes. Le tableau présente un résumé des huit SAÉ élaborées par les enseignantes.

Tableau 7 : Description des SAÉ

\begin{tabular}{|c|c|c|c|}
\hline \multicolumn{4}{|c|}{ SAÉ volet LECTURE 2010-2011 } \\
\hline $\begin{array}{l}\text { SAÉ préscolaire régulier } \\
\text { ( } 2 \text { enseignantes) }\end{array}$ & $\begin{array}{l}\text { SAÉ } 1^{\text {er }} \text { cycle régulier et adap- } \\
\text { tation scolaire } \\
\text { ( } 3 \text { enseignantes) }\end{array}$ & $\begin{array}{l}\text { SAÉ } 2^{\mathrm{e}} \text { cycle régulier et adap- } \\
\text { tation scolaire } \\
\text { ( } 3 \text { enseignantes) }\end{array}$ & $\begin{array}{l}\text { SAÉ } 1^{\text {er }} \text { et } 2^{\mathrm{e}} \text { cycles classe langage } \\
\text { ( } 3 \text { enseignantes } \\
\text { orthopédagogues) }\end{array}$ \\
\hline $\begin{array}{l}\text { Thème: la famille } \\
\text { Titre: Boucle d'or et les trois ours } \\
\text { Moyens concrets en lien avec la } \\
\text { littératie: } \\
\text { Discussions autour des activités } \\
\text { familiales des élèves; } \\
\text { La grandeur des vêtements: } \\
\text { petits, moyens ou grands; } \\
\text { Vocabulaire lié à la famille; } \\
\text { Livret de sa famille présenté aux } \\
\text { parents et à la classe. }\end{array}$ & $\begin{array}{l}\text { Thème: la publicité } \\
\text { Titre: La publicité, je ne me ferai } \\
\text { pas attraper! } \\
\text { Moyens concrets en lien avec la } \\
\text { littératie: } \\
\text { Techniques de vente; } \\
\text { Publicité télévisuelle et écrite; } \\
\text { Cadeau d'anniversaire. }\end{array}$ & $\begin{array}{l}\text { Thème: la poste } \\
\text { Titre: Voyage d'une lettre } \\
\text { Moyens concrets en lien avec la } \\
\text { littératie: } \\
\text { Conventions d'adressage de } \\
\text { différents courriers; } \\
\text { Parcours du courrier du destina- } \\
\text { teur au destinataire; } \\
\text { Familiarisation avec le milieu de } \\
\text { la poste. }\end{array}$ & $\begin{array}{l}\text { Thème: l'hiver } \\
\text { Titre: Les plaisirs d'hiver } \\
\text { Moyens concrets en lien avec la } \\
\text { littératie: } \\
\text { Les conditions météorologiques; } \\
\text { Les activités d'hiver (faire un bon- } \\
\text { homme de neige); } \\
\text { Les vêtements et les accessoires } \\
\text { d'hiver; } \\
\text { Les circulaires, les revues, les catalo- } \\
\text { gues. }\end{array}$ \\
\hline
\end{tabular}

\begin{tabular}{|c|c|c|}
\hline \multicolumn{3}{|c|}{ SAÉ volet ORAL 2010-2011 } \\
\hline $\begin{array}{l}\text { SAÉ préscolaire régulier, } 1^{\text {er }} \text { cycle } \\
\text { régulier et } 1^{\text {er }} \text { cycle adaptation } \\
\text { scolaire } \\
\text { ( } 4 \text { enseignantes) }\end{array}$ & $\begin{array}{l}\text { SAÉ } 1^{\text {er }} \text { cycle régulier et adaptation scolaire } \\
\text { ( } 3 \text { enseignantes) }\end{array}$ & $\begin{array}{l}\text { SAÉ } 1^{\text {er }} \text { et } 2^{\mathrm{e}} \text { cycles classe langage } \\
\text { ( } 3 \text { enseignantes } \\
\text { orthopédagogues) }\end{array}$ \\
\hline $\begin{array}{l}\text { Thème: les métiers } \\
\text { Titre: Quand je serai grand, je serai... } \\
\text { Moyens concrets en lien avec la littératie: } \\
\text { Présentation orale de différents métiers par } \\
\text { l'entremise de certains invités et de lectures } \\
\text { animées sur la thématique. } \\
\text { Visite du Musée des enfants qui permet de faire } \\
\text { vivre plusieurs métiers aux enfants. Organi- } \\
\text { sation d'une foire aux métiers dans laquelle } \\
\text { les élèves présenteront oralement le métier } \\
\text { de leur choix. Présentation orale du cahier du } \\
\text { métier des enfants de la classe à la maison. }\end{array}$ & $\begin{array}{l}\text { Thème: la lecture à voix haute à d'autres } \\
\text { personnes } \\
\text { Titre: Raconte-moi une histoire à haute voix et je } \\
\text { t'en raconterai une à mon tour } \\
\text { Moyens concrets en lien avec la littératie: } \\
\text { Visite de quelqu'un en classe (grands-parents, } \\
\text { bibliothécaire, parents) qui vient lire à voix } \\
\text { haute une histoire collective aux élèves de la } \\
\text { classe. } \\
\text { Lecture collective animée enregistrée sur } \\
\text { disque compact, en mettant en pratique les } \\
\text { techniques apprises (stratégies de lecture à } \\
\text { voix haute) (offert aux parents). }\end{array}$ & $\begin{array}{l}\text { SAÉ 1er et 2e cycles classe langage } \\
\text { ( } 3 \text { enseignantes orthopédagogues) } \\
\text { Thème: les peurs } \\
\text { Titre: J'ai peur un peu, beaucoup, des fois... } \\
\text { Moyens concrets en lien avec la littératie: } \\
\text { Écoute de chansons sur les peurs } \\
\text { Visionnement de films pouvant susciter des } \\
\text { peurs chez l'enfant } \\
\text { Visite de la bibliothèque pour emprunter diffé- } \\
\text { rents livres sur les peurs. } \\
\text { Causerie sur le thème de la peur } \\
\text { Sondage oral sur les peurs dans les classes de } \\
1^{\text {er }} \text { cycle }\end{array}$ \\
\hline
\end{tabular}




\section{Avantages et limites de la recherche-action-formation}

Les avantages de la recherche-action-formation nous semblent plus nombreux que ses inconvénients. Ce type de recherche permet de mettre réellement en pratique une formation donnée sur une période de temps assez longue, en ce sens que les participants ont le temps de recevoir l'information, de la comprendre, de la mettre en pratique dans leur classe et de revenir en séminaire discuter de leurs bons coups et de leurs difficultés, mais également de leur compréhension ou de leur incompréhension du concept à l'étude. Le fait de travailler avec des enseignantes sur un concept et de l'approfondir sur deux années de recherche a un effet certain sur le changement de leurs pratiques, ici en littératie volets lecture et oral, comme le précisent Paillé (1994), Marcel (1999), Charlier (2005), Lafortune (2006) ainsi que Prud'homme et coll. (2011). Également, concevoir une formation en équipe interdisciplinaire université-milieu (directeur d'école, conseillères pédagogiques, orthopédagogue) est une grande force de notre projet. En effet, les forces de tous s'unissent autant dans la conception, dans l'animation, dans la préparation que dans la définition des concepts à l'étude. Chaque membre de l'équipe conceptrice, avec ses forces, était présent pour soutenir les enseignantes en tout temps. Elles se sont senties épaulées, guidées, encadrées, comme le propose Paillé (1994), et ont mené leur SAÉ à terme avec brio. De plus, la formule pédagogique choisie pour la formation, soit le séminaire, a été une formule gagnante, car elle a permis aux enseignantes de vivre des conflits cognitifs nécessaires aux changements de pratiques, d'échanger avec des collègues d'autres écoles, de partager des expériences, de répondre à leurs besoins pédagogiques et de formation continue tel qu'expliqué en problématique. Finalement, les nombreuses retombées de la recherche-action-formation sont un avantage indéniable pour les participantes : création d'un matériel didactique inédit - des SAÉ en lecture et en oral intégrant la littératie -, participation à des communications dans des congrès professionnels courus par les enseignantes, participation à la rédaction d'articles dans des revues professionnelles et formation continue ayant permis un changement des pratiques de lecture et d'oral des enseignantes.

Deux limites de ce type de recherche peuvent être identifiées : le degré d'adhésion des participants à une démarche longue qui les pousse à sortir de leur zone de confort, et l'engagement réel des participants dans un projet qui propose des objectifs parfois loin de ce qu'ils vivent ou souhaitent vivre. Par exemple, comme le soutient Lafortune (2006), offrir une formation théorique à des gens qui souhaitent plutôt recevoir du matériel utilisable de manière rapide dans leurs classes peut être un frein à l'engagement de certaines personnes. Un autre inconvénient peut aussi être de ne pas donner la formation dans le milieu, soit dans les écoles. Quoi qu'il en soit, comme le dit Paillé (1994), ces

obstacles ne sont certes pas insurmontables puisque notre expérience est largement positive. Une autre limite reliée à notre projet de recherche est que les changements positifs observés chez les élèves par les enseignantes sont seulement basés sur leurs perceptions et appréciations ainsi que sur les coconstructions des chercheurs et des praticiennes. 


\section{Conclusion}

Nous avons avant tout souhaité, dans notre article, mieux définir ce qu'est la recherche-action-formation en illustrant notre propre expérience. Nous avons également voulu illustrer la complexité d'un type de recherche démontrant une double tension entre la construction de l'explication scientifique et la construction du savoir (Paillé, 1994). Voilà pourquoi nous avons présenté nos outils méthodologiques : démontrer que la recherche-action-formation est rigoureuse, qu'elle suit un désign méthodologique à la fois souple et complexe, qu'elle permet de recueillir une quantité impressionnante de données sur les pratiques effectives des enseignantes et sur le renouvèlement de leurs pratiques. Nous croyons avoir pu démontrer la pertinence sociale de ce type de recherche. La recherche-action-formation est développée « [...] en éducation et pour l'éducation », et montre bien que « [...] le chercheur est acteur, ici du développement organisationnel, là de l'intervention sociale, ailleurs de la formation » (Paillé, 1994, p. 226). 


\section{Références}

Brodeur, M., Dion, É., Laplante, L., Mercier, J., Desrochers, A. et Bournot-Trites, M. (2011). Prévenir les difficultés d'apprentissage en lecture: mobilisation des connaissances issues de la recherche par l'implantation du modèle à trois niveaux. Vivre le primaire, 23(1), 30-32.

Charlier, B. (2005). Parcours de recherche-action-formation. Revue des sciences de l'éducation, XXXI(2), 259-272.

Coghlan, V. et Thériault, J.-Y. (2002). L'apprentissage du français en milieu minoritaire, une revue documentaire. Ottawa : Centre interdisciplinaire de recherche sur la citoyenneté et les minorités en partenariat avec la Fédération canadienne des enseignantes et des enseignants.

Dion, É., Brodeur, M., Gosselin, C., Campeau, M.-Ė. et Fuchs, D. (2010). Implementing Research-Based Instruction to Prevent Reading Problems among Low-Income Students: Is Earlier Better? Learning Disabilities Research \& Practice, 25(2), 87-96.

Dolbec. A. et Clément, J. (2000). La recherche-action. Dans T. Karsenti et L. Savoie-Zajc (dir.), Introduction à la recherche en éducation (p. 199-224). Sherbrooke : Éditions du CRP.

Dumais, C. (2011). L'oral pragmatique : un enseignement de l'oral près de la réalité des élèves. Vivre le primaire, 24(1), 42-44.

Gauthier, A. (2010). Proposition de composantes d'une situation d'apprentissage et d'évaluation (SAÉ) modèle en français écrit à la première année du deuxième cycle du secondaire (Mémoire de maitrise inédit). Université du Québec en Outaouais.

Grenier, S., Jones, S., Strucker, D. J., Murray, S. T., Gervais, G. et Brink, S. (2008). Enquête internationale sur l'alphabétisation des adultes. L'apprentissage de la littératie au Canada: Constatations tirées de l'Enquête internationale sur les compétences en lecture. Ottawa : Statistique Canada Ressources humaines et Développement social Canada.

Haager, D., Klingner, J. et Vaughn, S. (2007). Evidence-Based Reading Practices for Response to Intervention. Baltimore, États-Unis : Paul H. Brookes Publishing.

Karsenti, T. et Savoie-Zajc, L. (2004). La recherche en éducation : étapes et approches. Sherbrooke : Éditions du CRP.

Lafontaine, L. (2011). Activités de production et de compréhension orales. Présentation de genres oraux et exploitation de documents sonores. Montréal : Chenelière.

Lafontaine, L. et Préfontaine, C. (2007). Modèle didactique descriptif de la production orale en classe de français langue première au secondaire. Revue des sciences de l'éducation, XXXIII(1), 47-66.

Lafontaine, L., Dezutter, O. et Thomas, L. (2010). Constats d'enseignants du Nouveau-Brunswick francophone au sujet de la séquence didactique comme outil d'enseignement et d'apprentissage du français en milieu minoritaire. Éducation francophone en milieu minoritaire, 5(2). Repéré à http://www.reefmm.org/documents/v5n2lafontaine.pdf. 
Lafortune, L. (2006). Accompagnement-recherche-formation d'un changement en éducation : un processus exigeant une démarche de pratique réflexive. Formation et pratiques d'enseignement en questions, 5, 187-202.

Legendre, R. (2005). Dictionnaire actuel de l'éducation (3éd.). Montréal, Québec : Guérin.

Maltais, C. (2007). Relation entre la littératie familiale en milieu francophone et l'incidence d'un programme de maternelle quatre ans à temps plein sur le développement du langage et de la lecture des enfants. Éducation francophone en milieu minoritaire, 2(1), 6-18.

Manço, A. A. et Vaes-Harou, A. (2009). Accueil scolaire des immigrés non francophones dans le cycle secondaire en Communauté française de Belgique : enseignements d'une recherche-formation. Didactica, Lengua y Literatura, 21, 227-253.

Marcel, J.-F. (1999). La démarche de recherche-formation. Proposition pour un trait d'union entre la recherche et la formation dans le cadre de la formation continue des enseignants. Recherche et formation, 32, 89-100.

Maurer, B. (2001). Une didactique de l'oral du primaire au lycée. Paris, France : Bertrand Lacoste.

Ministère de l'Éducation, du Loisir et du Sport. (2007). Mieux soutenir le développement de la compétence à écrire. Rapport du Comité d'experts sur l'apprentissage de l'écriture. Québec, Québec: Gouvernement du Québec.

Ministère de l'Éducation, du Loisir et du Sport. (2008). Rapport du Comité d'experts sur l'apprentissage de l'écriture. Québec: Gouvernement du Québec.

Ministère de l'Éducation, du Loisir et du Sport. (2009). Guide pour l'appel de projets Programme de soutien à la formation continue du personnel scolaire. Québec: Gouvernement du Québec.

Paillé, P. (1994). Pour une méthodologie de la complexité en éducation : le cas d'une recherche-action-formation. Revue canadienne de l'éducation, 3(19), 215-229.

Prud'homme, L., Dolbec, A. et Guay, M.-H. (2011). Le sens construit autour de la différenciation pédagogique dans le cadre d'une recherche-action-formation. Éducation et francophonie, XXXIX(2), 165-188.

Prud'homme, L. et Guay, M.-H. (2011). La recherche-action. Dans T. Karsenti et L. Savoie-Zajc (dir.), La recherche en éducation: Étapes et approches (3e éd.) (p. 183-211). Montréal : ERPI.

Réseau canadien de recherche sur le langage et l'alphabétisation (2009). Stratégie nationale d'alphabétisation précoce. London, Ontario : Réseau canadien de recherche sur le langage et l'alphabétisation.

Riente, R. (2010). Activités d'apprentissage, SAÉ et SÉ. Quelques précisions. Québec français, 158, 5253.

Veyrunes, P., Bertone, S. et Durand, M. (2003). L'exercice de la pensée critique en recherche-formation: vers la construction d'une éthique des relations entre chercheurs et enseignants débutants. Savoirs, 2(2), 51-70. 
TITRE: INTÉGRATION DE LA MULTIMODALITÉ DANS LES PROJETS D'INNOVATION DE STAGIAIRES EN FORMATION

Auteur(s): Nathalie Lacelle, Ph.D., UQAM et Amal Boultif, ChARgÉE de COURS, UQAM

PubliCATION: LA RECHERCHE-ACTION ET LA RECHERCHE-DÉVELOPPEMENT AU SERVICE DE LA LITTÉRATIE.

PAGES: $68-96$

DiRecteurs: ISABElle CARIgnan, MARIE-Christine BEAUdRY ET FrançoIs LAROSE

ÉdiTEUR: LES ÉdITIONS DE L'UNIVERSITÉ DE SHERBROOKE, 2016.

ISBN: 978-2-7622-0354-7

URI: HTTP://HDL.HANDLE.NET/11143/8814

DOI: HTTP://DX.DOI.ORG/10.17118/11143/8814 


\section{Intégration de la multimodalité dans les projets d'innovation de stagiaires en formation}

Nathalie Lacelle, Ph.D., UQAM

Amal Boultif, chargée de cours, UQAM

Résumé : L'article présente une recherche-action-formation menée auprès de seize étudiants en formation pratique qui ont eu à intégrer des pratiques multimodales à leur projet d'innovation pédagogique. Nous décrivons et analysons les modalités d'accompagnement des stagiaires impliqués afin de déterminer comment ces dernières ont permis le transfert de connaissances en recherche dans la pratique enseignante grâce à un accompagnement universitaire inspiré du nouveau paradigme de la multimodalité. Nous nous appuyons essentiellement sur les bilans réflexifs des stagiaires qui consignent leurs analyses quant à l'intérêt et à l'efficacité du recours à des pratiques d'enseignement multimodales pour résoudre des difficultés d'apprentissage disciplinaires de leurs élèves lors des stages. En ce sens, les connaissances produites sont à la fois méthodologiques et praxéologiques. Enfin, nous proposons des pistes de réflexion sur l'apport de la littératie médiatique et des TIC sur l'enseignement des disciplines et sur la manière dont la recherche peut aider les enseignants en formation à innover afin de développer des pratiques d'enseignement en phase avec les modes de communication modernes.

Mots-clés : littératie médiatique multimodale, projets d’innovation, stagiaires, formation initiale. 


\section{Introduction}

Les recherches récentes visant à renouveler les approches didactiques en lecture/écriture sur des supports variés, intégrant à différents degrés l'image, le texte et le son, soutiennent la nécessité de passer du paradigme de la littératie classique (monomodale) à celui de la littératie médiatique ${ }^{1}$ (multimodale) en éducation (Lebrun, Lacelle et Boutin, 2012a, 2012b). Or, un tel changement dans les conceptions et les pratiques des enseignants passe assurément par l'intégration de ces nouvelles approches dans la formation universitaire et la formation continue. Dans le cadre de cet article, qui remet en question le transfert de la recherche dans la formation supérieure, nous présenterons la mise en œuvre d'une recherche-action-formation auprès de seize étudiants ${ }^{2}$ en formation pratique qui ont eu à intégrer des pratiques multimodales au sein de leurs projets d'innovation pédagogique. Nous décrirons et analyserons les modalités d'accompagnement des stagiaires impliqués et nous tenterons de déterminer comment ces dernières ont permis le transfert de connaissances en recherche dans la pratique enseignante. Nous nous appuierons essentiellement sur les bilans réflexifs des stagiaires qui consignent leurs analyses quant à l'intérêt et à l'efficacité du recours à des pratiques d'enseignement multimodales pour résoudre les difficultés d'apprentissage disciplinaires de leurs élèves lors des stages. En ce sens, les connaissances produites seront à la fois méthodologiques et praxéologiques. Enfin, nous proposerons des pistes de réflexion sur l'apport de la multimodalité, de la littératie médiatique et des technologies de l'information et de la communication (TIC) à l'enseignement des disciplines et sur la manière dont la recherche peut aider les enseignants en formation à innover afin de développer des pratiques d'enseignement en phase avec les modes de communication modernes. Deux questions fondamentales sous-tendent cette recherche: comment la recherche-action-formation favorise-t-elle le transfert de connaissances issues de la recherche à la pratique de stagiaires en formation? Quelles sont les incidences de ce transfert sur le développement de nouvelles pratiques didactique?

\footnotetext{
1. «Le concept de «littératie médiatique» (media literacy), auquel on accole le qualificatif de « multimodale » se répand de plus en plus, particulièrement en français. II s'agit d'une acception qui recoupe à la fois l'« éducation aux médias » (media éducation) et les usages multimodaux des médias dans des contextes diversifiés d'enseignement et d'apprentissage, en misant de façon spécifique sur les compétences en lecture/réception et en écriture/production avec les nouveaux médias, donc des compétences « textuelles » d'un nouveau genre.
}

2. Le stage IV s'inscrit dans le cadre du baccalauréat en enseignement secondaire. Ce stage d'internat à l'école secondaire s'effectue sous la supervision d'un enseignant associé. Le stagiaire y prend en charge toutes les responsabilités d'une tâche d'enseignement à des groupes classes et l'encadrement d'activités éducatives. Il s'agit essentiellement d'une période d'entrainement et d'expérimentation de sa future profession. 


\section{Problématique}

\section{Les enseignants et les TIC}

La littératie médiatique multimodale (LMM), exigeant très souvent la manipulation des TIC par les enseignants, il convient d'y intégrer des compétences technologiques. Or, une enquête réalisée auprès de 2065 futurs enseignants en stage au Québec portant sur une appréciation de chaque composante de la compétence professionnelle à intégrer les TIC (Villeneuve et coll., 2012) révèle que la compétence à « communiquer à l'aide d'outils multimédias variés » est la moins maitrisée. Une revue des recherches sur les pratiques enseignantes effectuée par Bussière et Gluszunski (2004) laisse supposer que ce n'est pas tant la disponibilité des TIC, mais la qualité et le type d'utilisation qui constituent un facteur-clé de l'apprentissage. Plusieurs chercheurs canadiens (Gauthier, 2006; Barrette, 2004; Bibeau, 2003) voient dans l'élaboration et l'expérimentation de scénarios didactiques en littératie médiatique la solution à une véritable mise en place de pratiques enseignantes intégrant les TIC. D’autres chercheurs anglais, américains et australiens (Walsh, 2008; Kress, 2003; Jewitt, 2002; Kress et coll., 2001; Kress et Van Leeuwen, 2001) insistent aussi sur la création en recherche de designs pédagogiques originaux utilisant des modes d'expression différents pour enrichir les curriculums actuels. Il apparait clairement que les enseignants ont besoin d'être accompagnés dans la création de scénarios didactiques qui s'appuient sur des pratiques multimodales, l'usage des TIC, et qui mobilisent chez les élèves des compétences en LMM.

Dans la pratique éducative, ceux (les enseignants) qui adoptent une plus large perspective sur les littératies et sur ce qui compte comme texte ne s'appuient pas uniquement sur la lecture de manuels scolaires et la production de copies écrites, mais ils amènent les élèves à voir comment les messages sont communiqués par plusieurs moyens, y compris les représentations dramatiques, les posters, les photos, les graphies, les images, les sites web, les CD-ROM, les DVDs, les jeux vidéos, la production vidéo et les autres outils des technologies numérisées (Street et coll. 2009; Cope et Kalantzis, 2000). (Dagenais, 2012, p. 25)

Les TIC se révèlent donc efficaces lorsqu'elles s'intègrent à une didactique diversifiée qui articule finement les activités d'apprentissage disciplinaires, les approches pédagogiques et les supports multimodaux. 


\section{Les TIC et la multimodalité en formation universitaire}

La présente recherche s'intéresse principalement aux composantes suivantes: communiquer à l'aide d'outils multimédias variés (3); utiliser efficacement les TIC pour rechercher, interpréter, communiquer de l'information et pour résoudre des problèmes (4); aider les élèves à s'approprier les TIC, à les utiliser pour faire des activités d'apprentissage, à évaluer leur utilisation de la technologie et juger de manière critique les données recueillies sur les réseaux (6). Le recours à la multimodalité (l'image, la vidéo, les cartes conceptuelles, les fichiers audios) par l'usage des technologies est ponctuellement recommandé, mais jamais dans une perspective de recours à la LMM pour s'approprier des contenus disciplinaires.

\section{Les objectifs de la recherche}

Dans le cadre de cette recherche, nous nous intéressons aux pratiques d'enseignement-apprentissage qui concernent l'usage de la multimodalité (combinaisons de modes sémiotiques et de médias) à travers des scénarios didactiques qui intègrent les multitextes (blogues, BD, vidéos, jeux vidéos, etc.). Nous proposons des outils méthodologiques (recherche) et didactiques (pratique) pour favoriser le recours à la multimodalité en enseignement des disciplines ${ }^{4}$ et aux compétences en LMM pour l'apprentissage. L'objectif général de cette recherche-action-formation vise à documenter le développement de pratiques multimodales d'enseignement-apprentissage de contenus disciplinaires par des enseignants stagiaires. Ces derniers devaient identifier un problème de départ, anticipé ou constaté chez leurs élèves, à la suite duquel ils devaient bâtir un projet pédagogique innovant intégrant la multimodalité et les TIC.

\section{Le modèle du TPaCK de Koehler et Mishra et la grille en LMM de Lebrun, Lacelle et Boutin}

Deux grilles de lecture, issues du cadre théorique des littératies médiatiques multimodale en contexte pédagogique, nous ont servi d'outils d'analyse des projets d'innovations des stagiaires sous deux angles complémentaires : celui des usages de la technopédagogie au moyen du modèle TPaCK

3. La multimodalité se caractérise par la présence de différents modes iconiques, linguistiques, gestuels et auditifs. Elle est toujours au moins à deux niveaux : premièrement, on retrouve une juxtaposition ou combinaison de différents modes et, deuxièmement, ces mêmes modes ont une nature multimodale (une séquence vidéo, par exemple, comprend des images animées, une gestuelle et des sons, les trois étant livrés symbiotiquement). C'est l'articulation des modes d'expression qui fait en sorte que le texte multimodal prend forme; il en va de même pour la combinaison de médias qui exigent du lecteur qu'il tisse les liens entre les informations recueillies à partir de divers documents multimodaux (Kress, 2010; Foucher, 1998).

4. Au Québec, le guide utilisé auprès des enseignants du préscolaire-primaire et du secondaire date de 2001 (MEQ). II comporte douze compétences dont la huitième s'intitule «Intégrer les technologies de l'information et des communications aux fins de préparation et de pilotage d'activités d'enseignement- apprentissage, de gestion de l'enseignement et de développement professionnel » et est constituée de six composantes (MEQ, 2001). 
(Mishra et Koehler, 2006) et celui de la multimodalité au moyen d'une grille des compétences en LMM (Lebrun, Lacelle, Boutin, 2012a, 2012b).

\section{Le modèle du TPaCK de Koehler et Mishra}

Le modèle du TPaCK offre une analyse pertinente des usages des TIC en contexte d'enseignement-apprentissage (Schmidt et coll. 2009). Ses concepteurs (Mirsha et Koehler, 2006 et 2009) le situent dans le prolongement du modèle de Shulman (1986) qui ne comprenait que deux éléments de départ dans le domaine de l'enseignement-apprentissage : le savoir sur les contenus à enseigner et le savoir sur la pédagogie qui sous-tend ces enseignements (Pedagogical content knowledge). Les travaux de Herring, Mishra et Koehler $(2016)^{5}$ ont enrichi ce cadre en y intégrant la technologie (Technical Pedagogical Content Knowledge). Ce modèle intégrateur veut contrer la vision uniquement instrumentale qu'ont beaucoup d'enseignants usagers des TIC.

La figure 1 présente les combinaisons considérées dans le modèle d'intégration des savoirs technologiques aux savoirs pédagogiques et de contenu élaboré par Koehler et Mirsha 2008).

5. Voir aussi le site du TPACK http://www.tpack.org/ crée par Mirsha et Koehler qui regroupe tous les travaux du groupe des deux chercheurs.

6. PK+CK : mise en œuvre conjointe des savoirs pédagogiques et des contenus qui sont les connaissances nécessaires pour l'enseignement d'un certain contenu disciplinaire.

PK+TK : l'intersection technologie / pédagogie renvoie à la compréhension des différentes façons dont la technologie peut être utilisée dans un contexte pédagogique.

CK+TK : les savoirs de contenu disciplinaire ajoutés aux savoirs technologiques permettent à l'enseignant d'avoir des représentations de la manière dont le contenu disciplinaire peut être soutenu ou approfondi à l'aide de la technologie.

TK+PK+CK : désigne la convergence des trois domaines en interaction. Nous avons là le cœur du modèle TPaC; il présente « les habiletés à développer des expériences d'apprentissage signifiantes pour les élèves en intégrant de façon dynamique les aspects relevant des contenus disciplinaires, de la pédagogie et de la technologie». 
Figure 1 : Modèle TPaCK

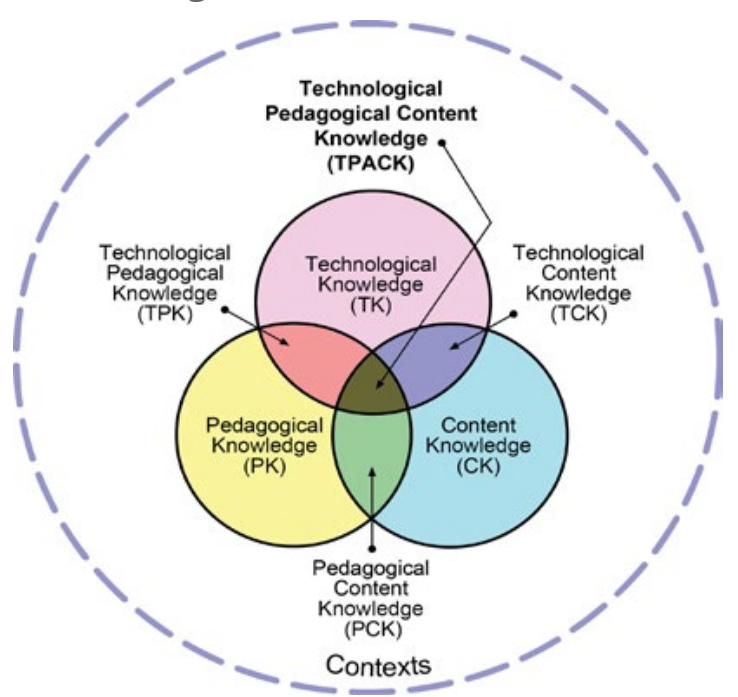

Image reproduite avec l'autorisation des éditeurs, (c) 2012 by tpack.org

Pour le présent article, nous nous sommes servies du modèle TPaCK $\mathrm{T}^{7}$ puisque nos questions de recherche étaient centrées sur les modalités d'accompagnement des stagiaires impliqués dans ce projet et sur les aspects praxéologiques qui touchent au transfert de connaissances en recherche dans la pratique enseignante.

\section{La grille en LMM de Lacelle, Lebrun, Boutin, Richard et Martel}

La grille transdisciplinaire en LMM (Lacelle et coll., 2015) vise essentiellement l'intégration de pratiques multimodales dans l'enseignement et l'apprentissage des disciplines. L'espace limité de cet article nous contraint à ne soumettre qu'une description sommaire de la grille de compétences en litmedmod ${ }^{8}$. Cette grille explicite les compétences cognitives et affectives (ex : décoder, comprendre et intégrer un message multimodal), les compétences pragmatiques générales (ex : reconnaitre et analyser les contextes de réception et/ou de production d'un message multimodal), les compétences sémiotiques générales (ex : reconnaitre/analyser/communiquer les signes ou symboles du message multimodal), la compétence modale spécifique (ex : reconnaitre/analyser/utiliser les ressources sémiotiques propres au mode visuel), la compétence multimodale (ex: reconnaitre/analyser/appliquer la simultanéité d'utilisation des codes, des modes, des langages et leurs modalités).

7. Figure 1

8. Pour consulter la grille complète : litmedmod.ca, onglet « outils». 


\section{La méthodologie : une recherche-action-formation en contexte de formation pratique des maitres}

Le paradigme de la multimodalité étant peu connu des enseignants ainsi que les compétences en littératie médiatique nécessaires aux apprenants pour accéder aux savoirs disciplinaires, nous avons choisi de recourir à une démarche de recherche-action-formation qui accentue le processus de la formation dans la recherche. La recherche-action-formation s'inscrit dans le sens d'une recherche pédagogique (Van der Maren, 2002) dont l'objectif premier est la fonctionnalité des savoirs produits à la fois pour l'action en classe et pour la formation des maitres (Prud'homme, 2007). Le chercheur-formateur doit donc considérer des enjeux à la fois scientifiques et pédagogiques dans l'élaboration du devis méthodologique.

\section{Les participants à la recherche}

Le projet de recherche a été initié et piloté par la chercheuse principale, Nathalie Lacelle, membre fondateur du groupe de recherche en littératie médiatique et multimodale LMM ${ }^{9}$, elle-même responsable d'un premier groupe de stagiaires $(\mathrm{N}=8)$. Une deuxième superviseure d'un deuxième groupe $(\mathrm{N}=8)$ avait la responsabilité de recueillir les rapports de stages et les documents utiles au projet. Une stagiaire doctorale ${ }^{10}$, Amal Boultif, spécialiste dans les usages pédagogiques des TIC, de la technologie à des fins d'enseignement et d'apprentissage de la lecture et de l'écriture, membre du groupe de recherche en Littératie Médiatique Multimodale (LMM), a dispensé aide et formation aux stagiaires et a participé à la collecte et à l'analyse des données.

Les sujets de la recherche, au nombre de seize, ont été recrutés dans les deux groupes composant les étudiants des séminaires des deux superviseures de stage, qui avaient la charge de former, d'encadrer et d'évaluer les apprentissages des futurs enseignants pour leur stage final d'internat en enseignement au secondaire. Au cours de ce stage, les stagiaires devaient prendre en charge toutes les responsabilités d'une tâche d'enseignement pendant douze semaines. Pour les fins de la recherche, et dans le but d'améliorer le développement de leur compétence technodidactique, ces derniers devaient élaborer un projet d'innovation pédagogique intégrant la multimodalité et l'expérimenter dans leurs classes respectives, soit de la $2^{e}$ à la $4^{e}$ secondaire. Des seize projets d'innovation, dix ont été jugés suffisamment complets pour les analyses de la recherche. Nous n'avons donc considéré, au final, pour la présente étude, que les projets finalisés et accompagnés des prétests ${ }^{11}$, des rapports du plan d'innovation et des bilans réflexifs qui s'y rattachent.

9. Groupe de recherche en littératie médiatique multimodale : http://www.litmedmod.ca/

10. Stage II du doctorat en éducation à l'Université du Québec à Montréal.

11. Nous explicitons ce point dans notre discussion. 


\section{Le design de la recherche}

Notre recherche a progressé en trois temps : le premier nous a permis de dispenser une formation aux stagiaires; le second, de documenter le travail des stagiaires et leurs usages de la multimodalité dans leurs classes durant la mise en œuvre du projet; enfin, le troisième, de recueillir les projets finalisés et les commentaires des enseignants stagiaires. Nous nous sommes concentrées, pour la présente recherche, à observer les modalités de mise en place de projets d'innovation pédagogiques en lien avec plusieurs disciplines du secondaire ${ }^{12}$ : cinq projets en français langue d'enseignement, trois projets en univers social et deux projets en mathématiques et sciences et technologie. Nous avons documenté les contenus des projets des stagiaires ( $\mathrm{N}=10)$ ainsi que leurs usages des TIC et des divers médias sollicités pour élaborer, mettre en œuvre, enseigner, consigner et conserver les traces des séquences d'enseignement apprentissage. Voici les principaux éléments qui composent le dispositif de la recherche ${ }^{13}$ :

- la formation dispensée par l'entremise des séminaires;

- les instruments de collecte de données : les questionnaires, les projets d'innovation et leurs plans de progression, les vidéos de présentation des projets et les journaux des chercheuses;

- le type de données (qualitatives et quantitatives) attendues et recueillies : bilans réflexifs des stagiaires et des chercheuses, réponses aux questionnaires (pratiques déclarées), présentations orales filmées des stagiaires (pratiques effectives) et bilans du stage;

- les instruments d'analyse prévus dans notre cadre théorique : le modèle TPaCK et la grille de compétences en LMM;

- indices de compréhension et d'intégration de la LMM, du TPaCK et portraits évolutifs des sujets de la recherche.

12. En conformité avec le programme de formation de l'école québécoise pour le secondaire et avec l'approche par projet qui recommande le développement de compétences transversales et disciplinaires en lien avec les usages des TIC et de la LMM.

13. Voir figure 2: design de la recherche. 
Figure 2 : Design de la recherche ${ }^{14}$

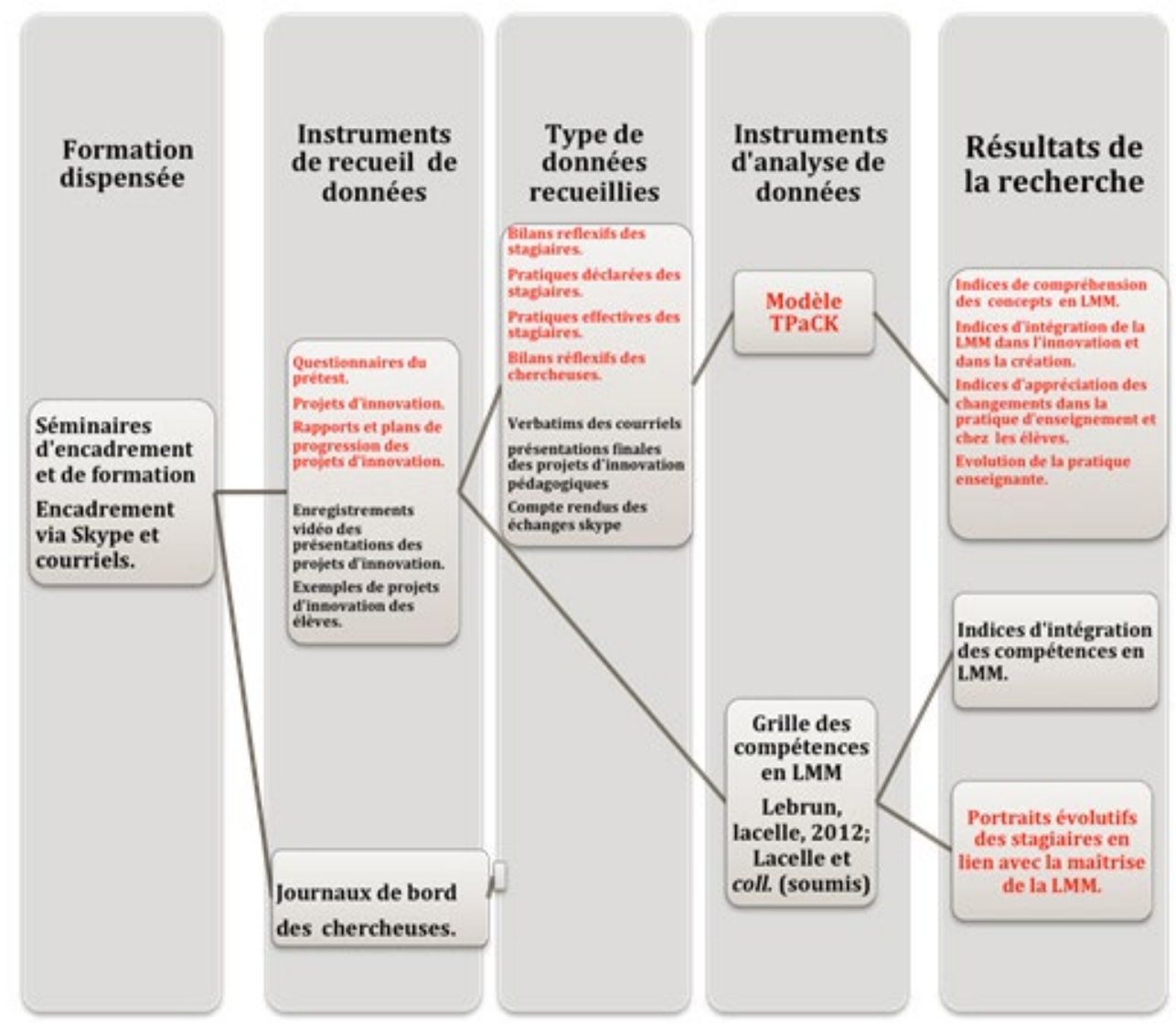

La phase de formation et modalités de suivis des enseignants stagiaires

L'équipe de chercheuses a conçu un document d'accompagnement (outils de collecte de données) pour les stagiaires leur permettant, dans un premier temps, d'évaluer les besoins de leurs élèves puis, dans un second temps, de déterminer les objectifs et les moyens pédagogiques et technologiques à mettre en place susceptibles d'y répondre.

L'annexe 2 présente un tableau qui fait la synthèse de la démarche d'encadrement et de formation de stagiaires, soit le nombre de stagiaires, le type d'encadrement et les objectifs des rencontres (séminaires et rencontres diverses), le nombre d'heures allouées à la formation et la nature de l'aide apportée aux stagiaires par l'équipe de recherche.

14. En rouge apparaissent les éléments développés dans le présent article. 


\section{Le traitement des données}

Les données recueillies correspondent à trois temps de notre recherche : le temps (1) qui précède la mise en œuvre pratique des projets d'innovation, qui touche plus particulièrement à la formation des stagiaires, et qui documente spécifiquement l'étendue des connaissances préalables des stagiaires et leurs compétences en lien avec la multimodalité; le temps (2) qui documente le cheminement des stagiaires et leur appropriation de la multimodalité et de ses pratiques effectives en contexte d'enseignement-apprentissage; enfin, le temps (3) qui documente la finalisation de projets d'innovation pédagogiques et l'évolution de la pratique de la multimodalité en contexte pédagogique chez les enseignants stagiaires. Comme les réponses aux questionnaires comportent des données chiffrées (questions fermées) et des questions à développement (questions ouvertes), cela implique un traitement qualitatif et quantitatif des données. Le codage des questions ouvertes ainsi que les commentaires du bilan réflexif figurant dans le rapport du plan de progression des stagiaires ont été saisis puis traités pour une analyse de contenu sémantique.

Le tableau 1 décrit les différents instruments de collecte de données, les moments documentés de la recherche (pré ou post expérimentation des stagiaires), le type de données qualitatives et quantitatives recueillies en fonction des objectifs de la recherche.

Tableau 1 : recueil de données et modalités de traitement

\begin{tabular}{|c|c|c|}
\hline \multicolumn{3}{|l|}{ Traitement des données } \\
\hline $\begin{array}{l}\text { Instruments de recueil } \\
\text { des données }\end{array}$ & Moments documentés & Thématiques et catégories recensées \\
\hline Questionnaires prétest & $\begin{array}{l}\text { Avant la mise en place des projets d'inno- } \\
\text { vation pédagogiques. }\end{array}$ & $\begin{array}{l}\text { Usages et pratiques autodéclarées par les stagiaires sur le degré de } \\
\text { compréhension initial de la LMM, le degré de maitrise autodéclaré } \\
\text { des usages de la multimodalité et des outils technologiques. }\end{array}$ \\
\hline $\begin{array}{l}\text { Plans de progression } \\
\text { Courriels }\end{array}$ & $\begin{array}{l}\text { Pendant la réalisation et la mise en } \\
\text { œuvre des projets d'innovation pédago- } \\
\text { giques }\end{array}$ & $\begin{array}{l}\text { Intégration des catégories du TPaCK et en particulier de leurs inter- } \\
\text { sections à la pratique enseignante des stagiaires }{ }^{1}\end{array}$ \\
\hline $\begin{array}{l}\text { Rapports de stage et } \\
\text { bilans réflexifs }\end{array}$ & $\begin{array}{l}\text { Pendant et à la suite de la mise en pra- } \\
\text { tique des projets d'innovation pédago- } \\
\text { giques }\end{array}$ & $\begin{array}{l}\text { Compréhension de la multimodalité à la suite de la mise en œuvre } \\
\text { des plans d'innovation pédagogiques. } \\
\text { Usages disciplinaires de la multimodalité. } \\
\text { Évolution des stagiaires vers la multimodalité et stabilisation des } \\
\text { pratiques. }\end{array}$ \\
\hline
\end{tabular}




\section{L'analyse des données}

Pour une analyse approfondie de notre corpus de données, nous avons adopté une démarche propre aux méthodes des approches qualitatives, et cela, avec l'intention de répondre à nos questions et objectifs de recherche. Nous avons adopté le principe de l'analyse documentaire (L'Écuyer, 1990). La catégorisation des unités de sens s'appuie sur les catégories prédéterminées suivantes qui seront sous-divisées à leur tour selon les concepts de notre grille sur les compétences en LMM. Catégorie 1 : indices de compréhension des enseignants des concepts en LMM. Catégorie 2 : indices d'appropriation des enseignants de la grille de compétences en LMM. Catégorie 3 : indices d'intégration des enseignants de la multimodalité dans la création et l'expérimentation de dispositifs d'enseignement. Catégorie 4 : indices d'appréciation des enseignants quant aux changements de pratiques pédagogiques mis en place en multimodalité et leur perception des retombées sur leurs élèves. Les indices ont été regroupés selon les disciplines et mis ensuite en commun afin de voir de quelle façon les enseignants des différentes disciplines se situent dans leur utilisation de la multimodalité et des TIC. En fin de parcours, les résultats ont également été analysés selon le processus de l'intégration de l'innovation de Charlier (2005; 2009) en revenant sur : 1- les conditions ayant favorisé l'implantation de la LMM comme innovation; 2- l'évolution des enseignants vers la LMM (de l'intérêt à l'engagement); 3- la stabilisation des pratiques de LMM (en prenant principalement comme base les journaux de terrain des chercheuses).

\section{La discussion sur les résultats}

Nous présenterons les résultats d'analyse des réponses des stagiaires aux questionnaires du prétest et des verbatims des bilans du plan de progression (commentaires réflexifs). Nous procèderons ensuite à une réorganisation de ces données à la lumière de nos grilles d'analyse, soit le modèle TPaCK et la grille de compétences en LMM. Nous avons organisé nos données selon qu'elles relevaient de pratiques déclarées (prétest) ou de pratiques réelles (rapports du plan de progression et bilans réflexifs) pour en extraire les thèmes prédéterminés et émergents.

\section{Les pratiques déclarées de la maitrise de la multimodalité par les stagiaires ${ }^{15}$}

L'histogramme (figure 3) montre que la majorité des stagiaires se jugent de « moyens » à « bons » à faire usage de divers supports de la multimodalité. On note aussi que la production de messages textuels et iconiques fixes est perçue par les stagiaires comme la mieux maitrisée alors que la manipulation d'autres modes d'expression (les images séquentielles (BD) ou mobiles et les messages sonores) l'est beaucoup moins. Ceci se confirme dans le graphique suivant (figure 4) où l'on note que les combinaisons modales semblent poser problème aux stagiaires surtout dans le cas d'images séquentielles, mobiles ou avec du son.

15. Traitement quantitatif d'une partie des données du prétest : 4 questions adoptant la modalité de l'échelle de Likert. 
Figure 3 : Compétences multimodales déclarées sur divers supports

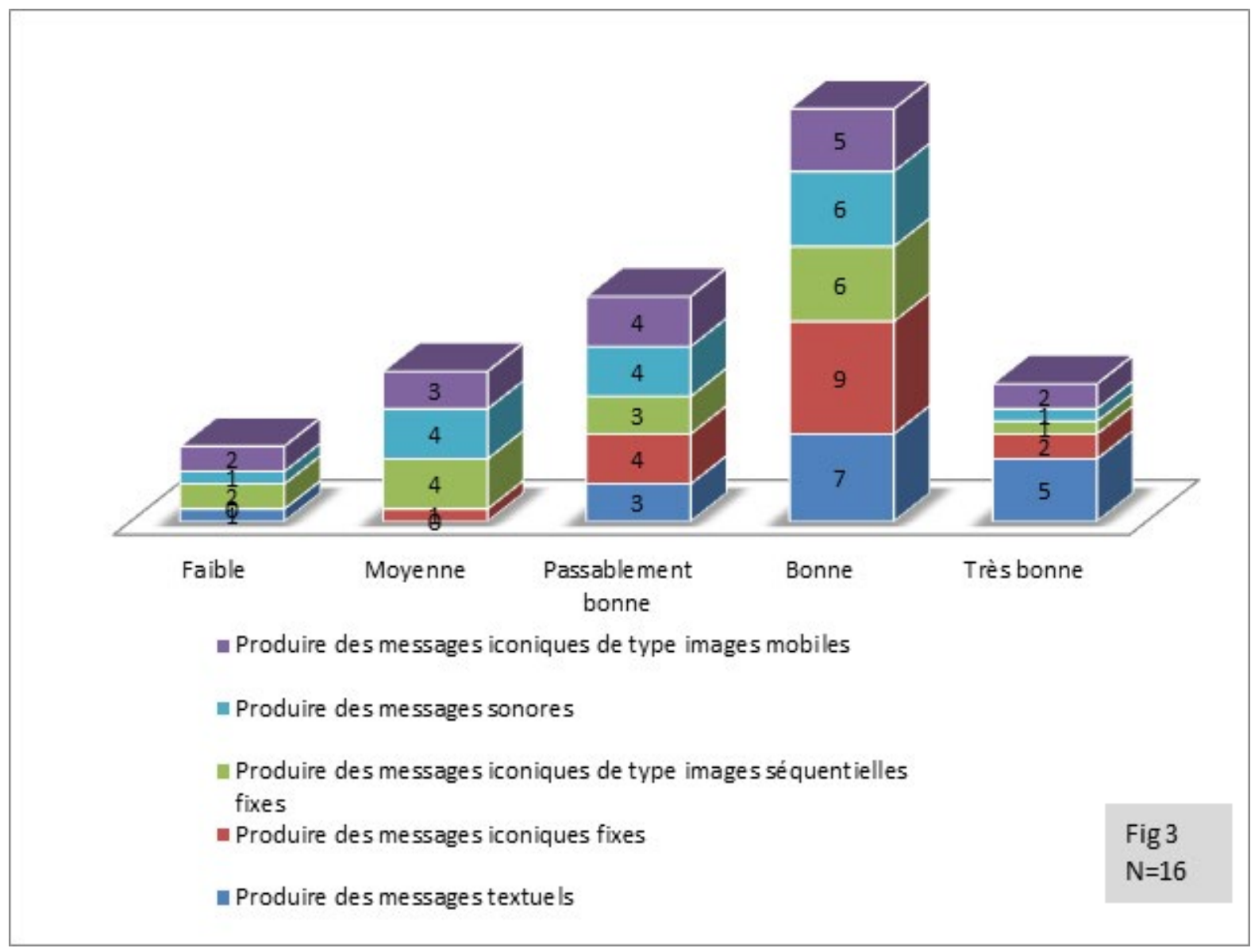


Figure 4 : Compétences déclarées à combiner la multimodalité en situation de production

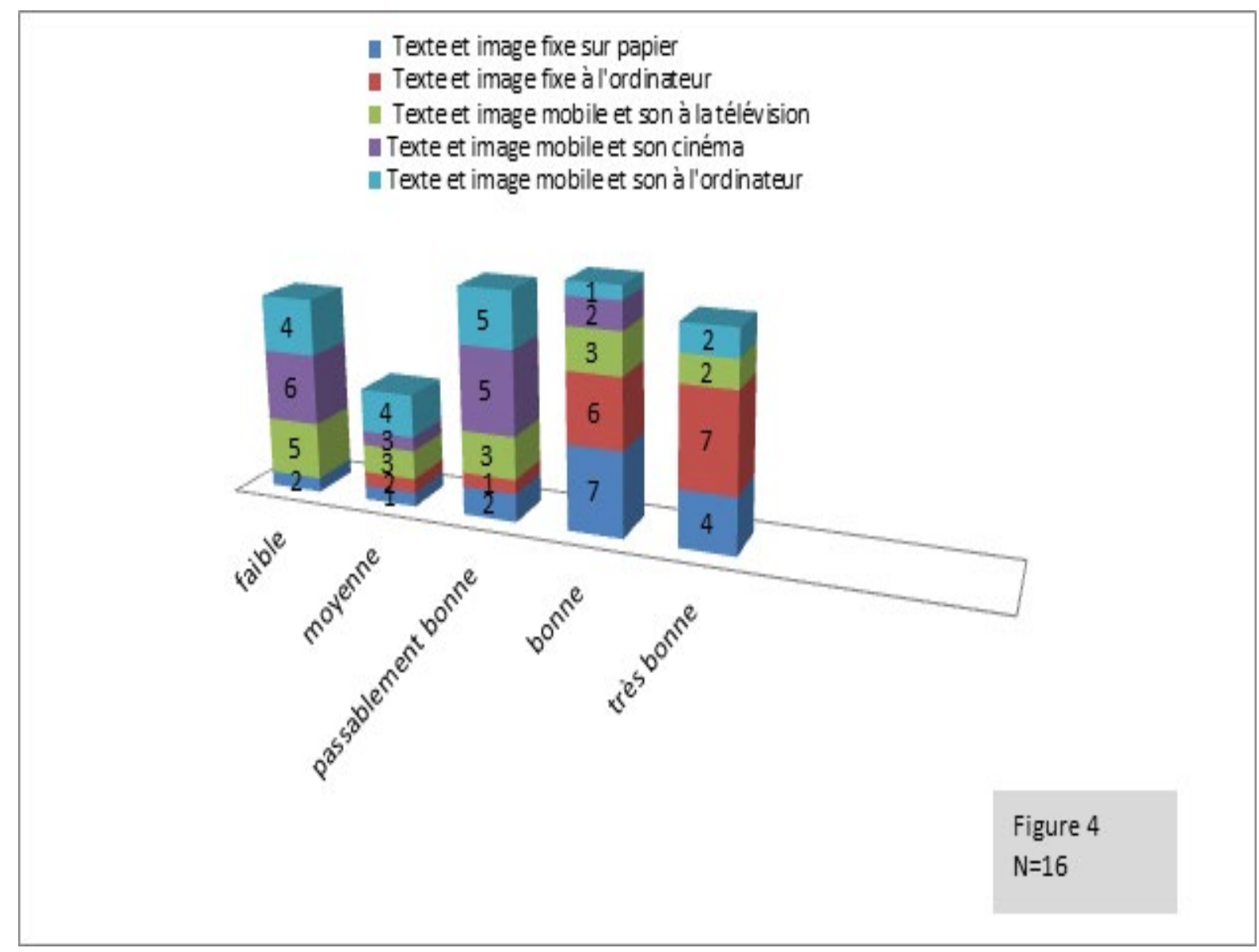

Les figures 5 et 6 s'appuient sur le traitement des données des questionnaires (catégorie des questions à développement) et illustrent l'importance accordée aux usages des outils technologiques chez les stagiaires (figure 5) ainsi que leur opinion sur l'utilité effective et l'apport de ces mêmes outils à leur pratique (figure 6). Les résultats montrent des indices très élevés d'appréciation des usages de la multimodalité chez les stagiaires qui cherchent à motiver leurs élèves qui baignent dans une culture multimodale et à varier leurs stratégies d'enseignement. Ce constat recoupe, encore une fois, les données des bilans réflexifs. 
Figure 5. Opinions des stagiaires sur l'importance de l'intégration d'outils technologiques à leur pratique pédagogique

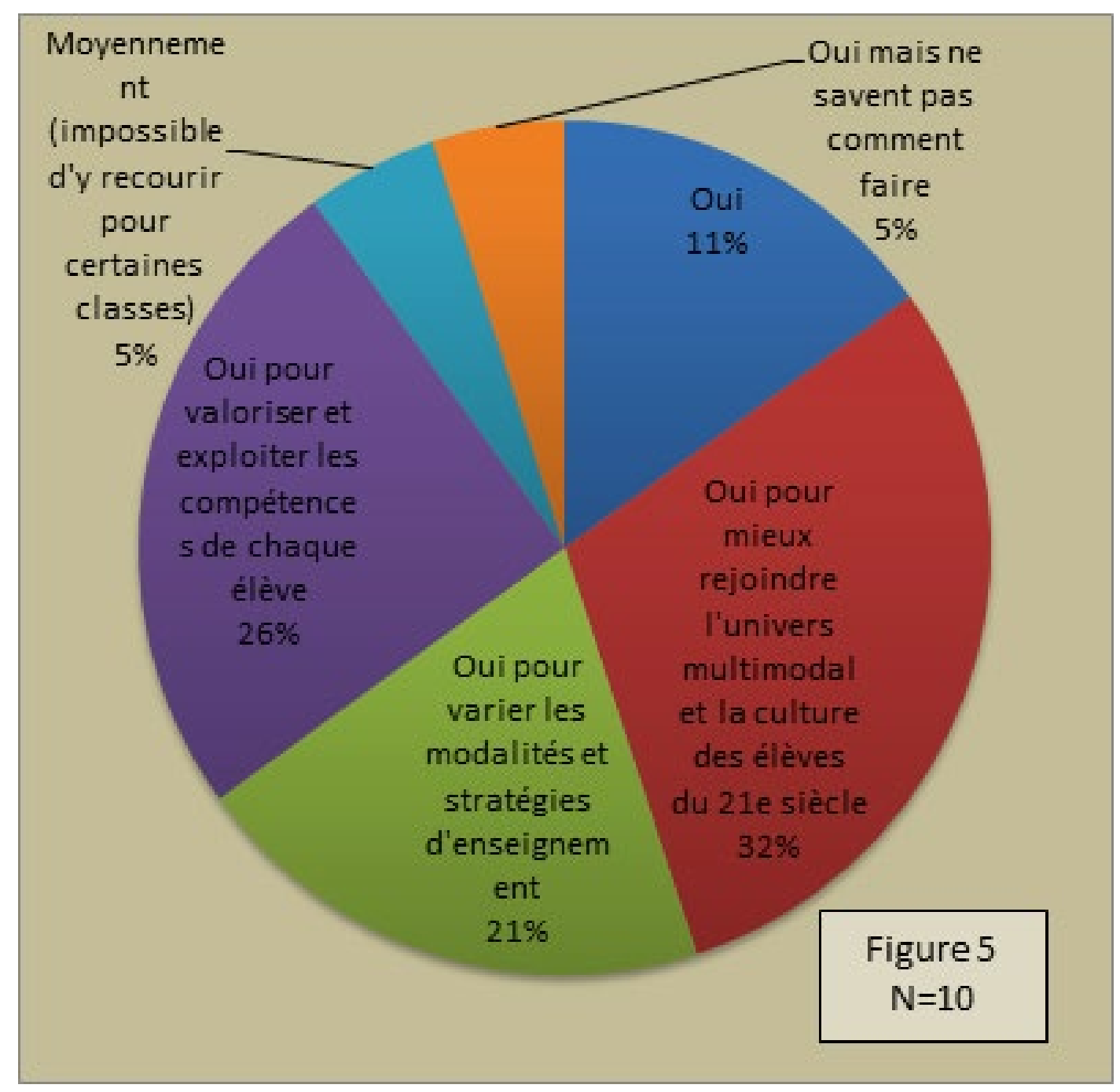


Figure 6: Opinion sur l'aide qu'apportent les supports multimodaux en enseignement-apprentissage

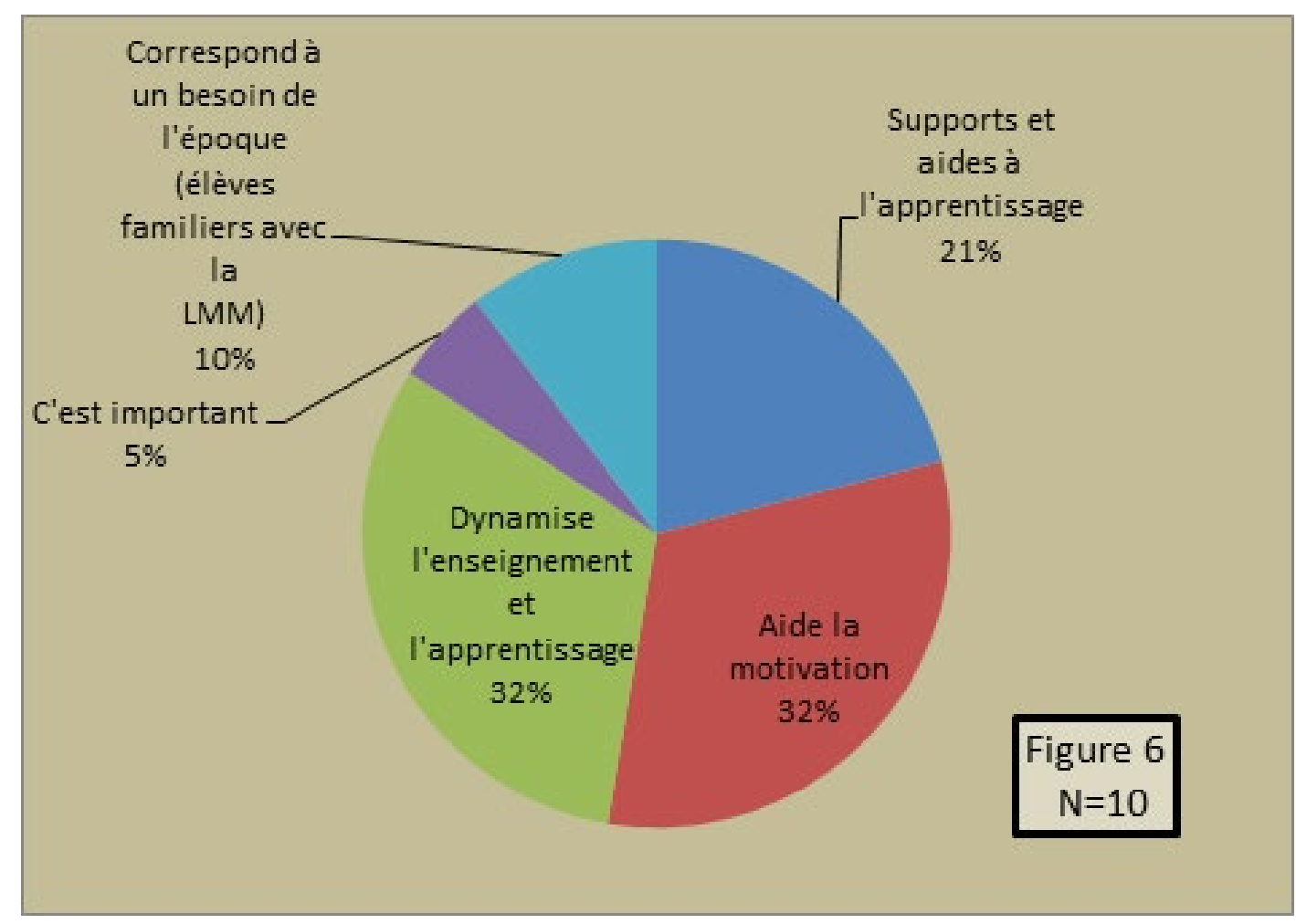

\section{La perception des retombées sur les élèves}

Dans leurs bilans, la majorité des dix stagiaires se déclarent satisfaits des objectifs atteints, mais ajoutent vouloir bonifier leurs projets afin de mieux combiner contenus, pédagogie et technologie. De plus, ils font état de changements chez les élèves, ce que des recherches sur les usages des TICE ont révélé, notamment la motivation et le rôle de la LMM dans l'appropriation du savoir (Loisier, 2011; Barette, 2004, 2005; Viau, 2005).

\section{Les indices de compréhension et d'appropriation des concepts en LMM}

Ces indices sont le fruit de la triangulation des éléments consignés par les étudiants dans leurs plans de progression du projet d'innovation, dans leurs bilans réflexifs (plan de progression du projet 
d'innovation ${ }^{16}$ ) et dans les réponses au questionnaire du prétes ${ }^{17}$. Notre analyse croisée montre une intégration très disparate de la LMM dans les projets d'innovation des dix stagiaires de notre échantillon. De plus, on remarque qu'il y a un écart entre les pratiques déclarées jugées assez moyennes et les usages réels qui sont faits de la LMM dans les projets de nos sujets qui révèlent une grande diversité d'usages.

Nous avons constaté, à la suite de l'analyse des projets, que cinq stagiaires ont eu plus de mal à arrimer tous les aspects de la multimodalité à leur pratique enseignante ${ }^{18}$.

J'ai pu développer mon habileté à utiliser différentes modalités pour la présentation de cours. C'est évident que j'appliquerais ce principe pour d'autres notions et activités. Cependant, je me rends compte, en complétant ce document, que je n'ai pas exploité les différentes modalités dans un objectif de développer des compétences chez les élèves par rapport à ces modalités. Donc, à l'avenir, j’essayerai de développer des compétences multimodales chez mes élèves. (Nora)

En effet, la multimodalité et les TIC sont surtout utilisées comme supports à l'enseignement. Les participants se sont limités à des usages des TIC pour l'enseignement (usage de logiciels et de supports et de ressources multimodales) sans nécessairement se pencher sur les compétences multimodales que les élèves doivent mobiliser pour s'approprier les savoirs disciplinaires. Et pour preuve, nous avons constaté l'absence d'évaluation des aspects relatifs à la multimodalité et à l'usage des TIC. Par opposition, nous remarquons que les cinq autres sujets, qui ont un score TPaCK élevé (figure 7) ont exploité tous les aspects collaboratifs et créatifs que peut offrir la multimodalité en enseignement et en apprentissage (les trois sphères du modèle y sont représentées) et ont, pour la plupart, intégré l'évaluation des usages de la multimodalité et des compétences en LMM à leurs projets.

Les indices d'intégration de la multimodalité dans la pratique des enseignants, selon le modèle TPaCK

Pour répondre à notre objectif de déceler des indices d'intégration de la multimodalité par les enseignants, nous avons établi un score TPaCK en partant des bilans réflexifs, des plans de présentation des projets d'innovation et des présentations finales des stagiaires. Nous avons accordé trois points pour les usages des trois dimensions en synergie dans les projets, deux points quand les seules dimensions étaient les savoirs pédagogiques et les savoirs relevant des contenus mis en œuvre et un seul point quand une seule dimension était présente.

Notre analyse des dix projets finalisés révèle une intégration partielle du modèle TPaCK par les stagiaires. En effet, cinq projets sur les dix révèlent des faiblesses quand il s'agit de considérer le

16. Voir annexe 3.

17. Administré à la fin des séminaires de formation.

18. Voir score TPaCK, supra. 
lien entre les trois pôles, soit les savoirs technologiques, les savoirs pédagogiques et les savoirs sur les contenus d'apprentissage. Ces faiblesses apparaissent quand on considère l'intersection entre le pôle technologique et le pôle pédagogique (TPK) et celui entre le pôle technologique et les contenus (TCK). Ce constat confirme les résultats des recherches antérieures qui font état des difficultés rencontrées par les enseignants à établir des liens entre les contenus à enseigner et les aspects pédagogiques et technologiques (Mishra et coll., 2009; Schmidt et coll., 2009). Le faible usage de dispositifs d'évaluation chez les enseignants croisant les compétences en LMM et en TIC (Béliveau, 2011) se confirme dans la présente recherche puisque deux seuls stagiaires y ont eu recours.

Figure 7 : Évaluation des projets d'innovation en fonction du score TPaCK.

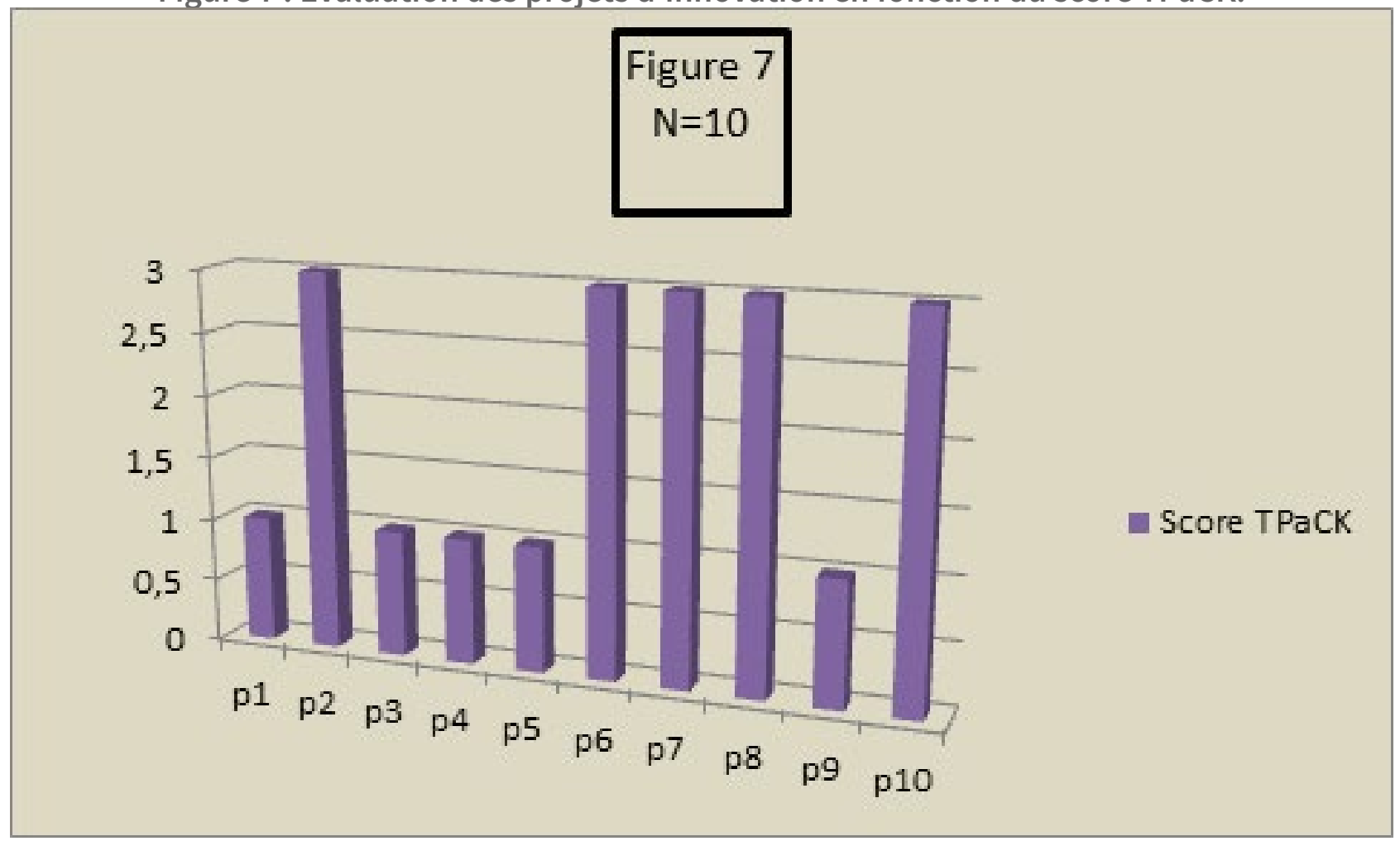

Bref, une forte tendance se dégage de nos observations : les stagiaires qui utilisent la technologie pour améliorer l'enseignement de leur discipline développent leur créativité et leur autonomie. C'est le cas des participants qui ont eu recours à des logiciels en ligne, ont réalisé un site web, ou encore ont produit une vidéo numérique d'apprentissage disciplinaire. Par exemple, un participant a réalisé un tutoriel sur support vidéo pour mieux aider ses élèves à comprendre un programme d'édition de textes en ligne.

\section{Indices d'évolution des pratiques des stagiaires}

La recherche-action-formation s'est avérée un déclencheur d'innovation des pratiques multimodales chez les participants. La formation reçue lors de séminaires universitaires a permis aux sta- 
giaires de vivre une véritable initiation à la multimodalité axée sur l'importance de créer des activités didactiques favorisant l'engagement des élèves et la mobilisation de compétences en LMM. Cela s'est confirmé dans plusieurs des projets que nous avons analysés.

Cette activité nous a permis d'échanger nos points de vue en plénière et ils (les élèves) ont aussi réalisé que le français n'est pas seulement de la grammaire, mais que tout peut devenir du français. Ils ont donc beaucoup apprécié les activités et le lien entre les élèves et moi est devenu plus fort à cause des différentes conversations que nous avons eues. (France)

Le dispositif de recherche mis en place nous a ainsi permis de réfléchir à l'impact de ce dernier sur les pratiques enseignantes des stagiaires. Nous avons aussi relevé que, de manière unanime, les stagiaires croient au potentiel motivant des TIC et au recours à des supports multimodaux en classe. Ils justifient ces usages par une pédagogie différenciée et variée appuyée par des méthodes d'enseignement-apprentissages diversifiées en lien avec la culture technologique des élèves. Ainsi, les stagiaires innovent en proposant des SAO (situation d'évaluation ouverte) adaptées aux usages de la LMM en contexte pédagogique.

J'avais envie de vivre une SAO (situation d'évaluation ouverte). Je trouve cette approche pédagogique intéressante pour la matière que j'enseigne. Je vois toutes les possibilités qui s'offrent à moi. (Paul)

Dans les projets, on note des indices de changement dans la pratique pédagogique des stagiaires qui s'appuient sur la diversification des outils technologiques des plateformes et des logiciels utilisés pour l'enseignement et l'apprentissage (figure 7). Les stagiaires citent les réseaux sociaux qui leur semblent utiles pour de futurs projets pédagogiques (Twitter, Wembly, etc.).

Concernant l'utilité de l'usage de la multimodalité en contexte pédagogique, notre analyse des bilans réflexifs et des questions à développement du questionnaire du prétest montre que presque tous les sujets expriment la nécessité d'en faire usage. Les arguments portent essentiellement sur le lien que la LMM permet d'établir avec les élèves comme moyen de les rejoindre (faire partie de leurs univers) et de les raccrocher (motiver) en faisant appel à leurs compétences informelles. Ainsi, à la question (2) du prétest:

"Comment l'utilisation des supports multimodaux peut-elle aider votre enseignement? », nous notons parmi les réponses:

Permettre aux élèves d'utiliser des supports avec lesquels ils sont familiers. (Paul)

Elle peut capter l'attention des élèves plus ou moins intéressés. Elle peut aussi permettre un intérêt chez quelques élèves. (Chantal) 
De plus, la multimodalité permettrait, selon les stagiaires, de varier les méthodes d'enseignement en diversifiant les stratégies, les outils et les activités proposées, et aussi de pouvoir s'adresser à tous les types d'apprenants en fonction de leurs intérêts, de leurs styles d'apprentissage et de leurs difficultés.

En allant rejoindre les diverses manières de comprendre chez les élèves (visuels, auditifs, kinésiques. (Myriam)

Varier les méthodes et approches en enseignement et introduire les aspects technologiques et multimodaux sont d'une grande aide pour les apprentissages des élèves. (Nora)

Dans la majorité des projets d'innovation, les participants ont fait preuve de créativité à toutes les étapes, soit lors de l'élaboration du projet d'innovation, du pilotage, des réajustements et de l'évaluation. Lors de la présentation finale des projets, nous avons noté chez les stagiaires un engagement manifeste, un investissement personnel (temps et moyens), une grande souplesse (adaptation à la situation), et ceci, malgré les contraintes des milieux d’accueil des stagiaires.

Notons aussi que, dans la partie «bilan » de projets d'innovation, les stagiaires ( $N=10)$ sont critiques quant aux usages diversifiés des outils multimodaux et à la gestion du temps.

Les bilans des dix stagiaires proposent aussi des pistes d'amélioration à leurs projets. Tous envisagent de reprendre leurs projets, mais soulignent le manque d'aide et de soutien technique.

Je pourrais le refaire en modifiant certaines équipes, en leur laissant le temps nécessaire pour réaliser le journal télévisé, possiblement leur faire confiance quant à filmer seuls dans l'école et sur son territoire extérieur, en changeant peut-être le cellulaire par un autre outil. (France)

\section{Les conditions pour la stabilisation des pratiques en LMM}

Lors des présentations finales des projets d'innovation et dans les bilans réflexifs, les stagiaires affirment avoir beaucoup appris sur leurs propres compétences en TIC et en LMM en contexte d'enseignement-apprentissage de leur discipline.

J'ai réalisé que j'étais davantage en mesure que je le pensais de concevoir des situations d'enseignement-apprentissage adaptées aux élèves et qui les intéressent. Aussi, intégrer les technologies n'a pas été un problème et même que j'ai trouvé que c'était beaucoup plus facile d'attirer l'attention des élèves. Je suis donc très fière de mon projet, même si je modifierais plusieurs choses si j'avais à le refaire. Il m’a permis de réaliser que je sous-estimais mes talents de création et que les projets de ce genre qui s'étalent sur plusieurs cours prennent beaucoup de temps à construire, mais qu'en fin de compte, ils sont très positifs et on gagne du temps à les faire.»(France) 
Durant le processus, j'ai réalisé certaines choses. Donc je vais modifier mon activité pour une prochaine fois. (Tania)

Comme nous le mentionnions plus haut, une difficulté majeure signalée par tous les stagiaires est le manque de collaboration et de soutien technique de la part des pairs et de certaines directions d'écoles (enseignants associés, professeurs ressources, personnel d'aide en technologie), et cela, en dépit du fait que la majorité des écoles qui ont accueilli les stagiaires étaient très bien dotées en termes de matériel et de locaux.

J'avais obtenu le soutien de quelques personnes pour faire les capsules. Malheureusement, au moment de filmer, pour le montage et la prise de son, plus personne n'était disponible. Je me suis retrouvé avec un éclairage médiocre et mon propre appareil photo numérique pour filmer. (Paul)

Les difficultés inhérentes aux usages de la technologique par des enseignants novices ont été rapidement compensées par les participants qui ont eu recours aux compétences en TIC et LMM de leurs propres élèves. On peut ainsi parler de co-construction des savoirs, dans un contexte hautement collaboratif, entre enseignants et élèves. Une des conditions de la stabilisation des pratiques d'intégration des TIC et de la LMM serait ainsi de concevoir une intégration des compétences informelles chez les élèves (ex. : maitrise technique des usages technologiques) dans l'élaboration même des scénarios didactiques avec l'enseignant.

Les réponses au posttest démontrent que malgré un sentiment de familiarité avec les TIC et les supports multimodaux, les participants ressentent une insécurité profonde à manipuler les modes et les supports en situation d'enseignement. Ainsi à la question : comment évaluez-vous votre compétence à utiliser la multimodalité dans vos cours?, les stagiaires ont répondu de la façon suivante: ".... oui mais, de façon restreinte et limitée » (p5) ; " Je me débrouille bien, cependant je suis moins à l'aise avec les cellulaires » (p9) ; "Je ne suis pas très à l'aise avec les outils » (p4). Les réponses aux questionnaires confirment leur réserve à devoir manipuler plusieurs outils technologiques et la LMM pour la production et l'édition vidéo ${ }^{19}$.

19. Voir figures 3, 4 et 7 pour la synthèse des résultats. 


\section{Conclusion}

L'avènement de nouvelles littératies qualifiées de multimodales auxquelles les apprenants ainsi que leurs enseignants sont de plus en plus confrontés pour accéder aux savoirs nous a amenées à réfléchir aux moyens d'intégrer la multimodalité et les TIC dans le champ de la didactique et dans celui de la formation des enseignants stagiaires. Actuellement, un grand nombre de ces jeunes enseignants stagiaires et les élèves auxquels ils font la classe sont des « digital natives » (Prensky, 2001) et se retrouvent forcément à manipuler des savoirs à l'aide de la multimodalité et des technologies. Cependant, à la lumière de notre recherche, on ne peut que déplorer une disparité et une pauvreté dans la formation effective à des usages harmonisés et efficaces de la technologie en classe, ce qui se remarque par les réticences et les craintes des participants à faire appel à la multimodalité pour innover dans leur enseignement des disciplines. Cela s'explique certainement par le manque de préparation des enseignants stagiaires en formation initiale, notamment dans les cours disciplinaires, et aussi par le cloisonnement de la compétence technologique dans les programmes de formation des maitres. De plus, nous attribuons aussi le peu de transfert de la recherche dans les pratiques enseignantes au manque de volonté des établissements scolaires qui n'ont pas encore pris la réelle mesure des recommandations émises par les recherches des dernières années sur la nécessité de soutenir les enseignants dans l'intégration des outils technologiques et multimodaux pour favoriser l'appropriation des savoirs disciplinaires. Ainsi, les enseignants qui s'essayent à des projets innovants se voient encore très peu encouragés, et les ressources mises à leur disposition sont déficitaires. Or, il apparait qu'une meilleure synergie entre la LMM, les TIC et les champs disciplinaires en formation initiale ouvre la voie à un renouvèlement des pratiques enseignantes en phase avec les recherches actuelles. Nous voyons ici des possibilités de collaborations plus étroites par le biais de la recherche-action-formation entre la recherche universitaire et la formation pratique à l'enseignement. Les avancées scientifiques viendraient ainsi soutenir les besoins des enseignants en formation identifiés à même leur expérience en classe. C'est une manière efficace de rendre les savoirs théoriques plus signifiants pour les enseignants en formation et peut-être de les motiver à poursuivre leur engagement dans la profession à travers la formation continue. 


\section{Références}

Barrette, C. (2004). Vers une métasynthèse des impacts des TIC sur l'apprentissage et l'enseignement dans les établissements du réseau collégial québécois. De la recension des écrits à l'analyse conceptuelle. Clic, 55, 8-15.

Béliveau, G. (2011). Analyse de recherches sur les TIC. Impact de l'usage des TIC au collégial. Rapport final. Département de philosophie du CÉGEP de Trois-Rivières. Québec. Repéré à http://philosophie.cegeptr.qc.ca/2011/08/analyse-des-recherches-sur-les-tice/

Bibeau, R. (2003). Taxonomie des contenus numérisés et des projets éducatifs avec les TIC. Direction des ressources didactiques, Ministère de l'Éducation du Québec. Repéré à http://eureka.ntic. org/file_download.php?lom_id=3033\&filename=taxonomie.pdf

Bussière, T. et Gluszynski, P. (2004). The impact of computer use on reading achievement of 15-yearolds. Learning Policy Directorate, Strategic Policy and Planning Branch Human. Resources and Skills Development. Canada.

Charlier, B. (2005). Parcours de recherche-action-formation. Revue des sciences de l'éducation, 31(2), 259-272.

Charlier, B. (2009). Réifier les pratiques d'enseignement: un enjeu de recherche et de formation. Dans F. Larose et A. Jaillet (dir.), Usages et mésusages de la trace informatique en enseignement et en recherche en éducation (p. 191-216). Paris : L'Harmattan.

Cope, B. et Kalantzis, M. (dir.). (2000). Multiliteracies. Literacy learning and the design of social futures, Londres : Routledge.

Dagenais, D. (2012). Littératies multimodales et perspectives critiques. Recherches en didactique des langues et des cultures: Les Cahiers de l'Acedle, 9(2), 5-46.

Foucher, A. L., (1998). Réflexions linguistiques et sémiologiques pour une écriture didactique du multimédia de la langue. ALSIC, 1(1) 3-25.

Gagnon, R. et Ziarko, H. (2011). Le texte informatif : un outil langagier pour apprendre. Québec français, 161, 50-52.

Gauthier, C. (2006). L'intégration des TIC dans les pratiques pédagogiques en milieu francophone minoritaire : tendances et défis, (collaboration de Mario Richard et Steve Bissonnette) : Fédération canadienne des enseignantes et des enseignants (FCE).

Gouvernement du Québec (2001). La formation à l'enseignement. Les orientations. Les compétences professionnelles. Québec : Ministère de l'Éducation.

Jewitt, C. (2002). The move from page to screen: the multimodal shaping of school. English. Visual communication, 1(2), 171-195. 
Koehler, M. J. et Mishra, P. (2008). Introducing TPACK. Dans AACTE Committee on Innovation \& Technology (dir.). Handbook of technological pedagogical content knowledge for educators (p. 3-29). New York : Routledge.

Kress, G. (2010). Multimodality: a social semiotic approach to contemporary communication. New York : Routledge.

Kress, G. et Van Leeuwen, T. (2001). Multimodal Discourses: The Modes and Media of Contemporary Communication. Londres: Edward Arnold.

Kress, G. et coll. (2001). Multimodal teaching and learning. The rhetorics of the science classroom. London (Continuum).

L'Écuyer, R. (1990). Méthodologie de l'analyse développementale des contenus. Méthode GPS et concept de soi. Québec: Presses de l’Université du Québec.

Lacelle, N., Lebrun, M., Boutin, J.-F., Richard M. et Martel, V. (2015). Les compétences en littératie médiatique multimodale au primaire et au secondaire: une grille d'analyse transdisciplinaire. Dans L. Lafontaine et J. Pharand (dir.), Littératie : vers une maitrise des compétences dans divers environnements (p. 168-184). Québec: Presses de l'Université du Québec,

Lebrun, M., Lacelle, N. et Boutin, J.-F. (2012a) (dir.). La littératie médiatique multimodale. De nouvelles approches en lecture-écriture à l'école et hors de l'école. Québec : Presses de l'Université du Québec.

Lebrun, M., Lacelle, N. et Boutin, J.-F. (2012b). Genèse et essor du concept de littératie médiatique multimodale. Dans Eli MacLaren (dir.), Mémoires du livre, Studies in Book Culture. Repéré à http://www.erudit.org/revue/memoires/2012/v3/n2/index.html

Loisier, J. (2011). Les nouveaux outils d'apprentissage encouragent-ils réellement la performance et la réussite des étudiants en FAD? Document préparé pour le Réseau d'enseignement francophone à distance du Canada. http://www.refad.ca/recherche/TIC/TIC_et_reussite_des_etudiants.pdf

Mishra, P. et Koehler, M. J. (2006). Technological Pedagogical Content Knowledge: A Framework for Teacher Knowledge. Teachers College Record, 108(6), 1017-1054.

Mishra, P., Koehler, M. et Harris, J. (2009). Teachers' Technological Pedagogical Content Knowledge and Learning Activity Types: Curriculum-based Technology Integration Reframed. Journal of Research on Technology in Education, 41(4), 393-416.

Herring, M. C., Mishra, P. et Koehler, M. J. (2016). Handbook of technological pedagogical knowledge (TPACK) for educators. New York: Routledge.

Prensky, M. (2001). Digital Natives, Digital Immigrants Part 1. (R. K. Belew et M. D. Vose, dir.) On the Horizon, 9(5), 1-6.

Prud'homme, L. (2007). La différenciation pédagogique: analyse du sens construit par des enseignantes et un chercheur-formateur dans un contexte de recherche-action-formation (Thèse de doctorat inédite). Université du Québec en Outaouais. 
Schmidt, D. A., Baran, E., Thompson, A. D., Mishra, P., Koehler, M. J. et Shin, T. S. (2009). Technological Pedagogical Content Knowledge (TPACK): The Development and Validation of an Assessment Instrument for Preservice Teachers. Journal of Research on Technology in Education, 42(2), 123149.

Shulman, L. S. (1986). Those who understand: Knowledge growth in teaching. Educational Researcher, 15(2), 4-14.

Street, B., Pahl, K. et Rowsell, J. (2009). Multimodality and New Literacy Studies. Dans J. Carey (dir.), The Routledge Handbook of Multimodal Analysis (p. 191-200). Londres : Routledge.

Van der Maren, J.-M. (2002). En quête d’une recherche pédagogique. Dans J. Donnay et M. Bru (dir.), Recherches, pratiques et savoirs en éducation (p. 89-104). Bruxelles : de Boeck et Larcier.

Viau, R. (2005). 12 questions sur l'état de la recherche scientifique sur l'impact des TIC sur la motivation à apprendre ». Université de Sherbrooke. Repéré à http://tecfa.unige.ch/tecfa/teaching/ LME/lombard/motivation/viau-motivation-tic.htm

Villeneuve, S., Karsenti, T., Raby, C. et Meunier, H. (2012). Les futurs enseignants du Québec sont-ils technocompétents? : une analyse en fonction de la compétence professionnelle à intégrer les TIC. Revue internationale des technologies en pédagogie universitaire, 9(1), 78-99.

Walsh, M. (2008). Worlds have collided and modes have merged: Classroom evidence of changed literacy practices. Literacy, 42(2), 101-108.

\section{Sitographie}

http://www.tpack.org/ 


\section{Annexes}

\section{Annexe 1}

Le tableau qui suit présente la liste des stagiaires et leurs projets par discipline enseignée.

\begin{tabular}{|c|c|c|c|}
\hline Projets & Discipline enseignée & Titre du projet & Classes et modalités de réalisation du projet \\
\hline Projet 1 & Français & L'histoire de l'humour & $\begin{array}{l}4 \text { classes de secondaire } 5 \text {. } \\
\text { En solo, en dyades et en grand groupe. }\end{array}$ \\
\hline Projet 2 & Français & Du populaire au littéraire & $\begin{array}{l}3 \text { groupes de secondaire } 2 . \\
\text { En équipes de } 4 \text { ou } 5 \text {. }\end{array}$ \\
\hline Projet 3 & Français & Les verbes pour conter & $\begin{array}{l}1 \text { classe de secondaire } 1 . \\
\text { En solo, en dyades et en grand groupe }\end{array}$ \\
\hline Projet 4 & Français & Les contes en rythme & $\begin{array}{l}1 \text { classe de secondaire } 3 . \\
\text { En solo, en dyades et en grand groupe. }\end{array}$ \\
\hline Projet 5 & Français & $\begin{array}{l}\text { La poésie et les jeux de mots de Marc Fa- } \\
\text { vreau et de Fred Pellerin }\end{array}$ & $\begin{array}{l}2 \text { groupes d'élèves de secondaire } 3 . \\
\text { En solo, en dyades et en grand groupe. }\end{array}$ \\
\hline Projet 6 & Sciences et technologie & L'anatomie humaine & $\begin{array}{l}1 \text { classe de secondaire } 3 . \\
\text { En science et technologie, en solo, en dyades et en } \\
\text { grand groupe }\end{array}$ \\
\hline Projet 7 & Univers social & Une image vaut mille mots & $\begin{array}{l}4 \text { groupes de secondaire } 1 . \\
\text { En solo. }\end{array}$ \\
\hline Projet 8 & Univers social & Les stars de la Renaissance & $\begin{array}{l}4 \text { groupes de secondaire } 2 . \\
\text { En solo, en dyades, autres modalités. }\end{array}$ \\
\hline Projet 9 & Univers social & Exposé des mouvements de pensée & $\begin{array}{l}6 \text { classes de secondaire } 4 . \\
\text { En solo, en dyades et en grand groupe. }\end{array}$ \\
\hline Projet 10 & Maths et physique & L’univers matériel en résumé. Maths & $\begin{array}{l}1 \text { classe de secondaire } 4 . \\
\text { En dyades. }\end{array}$ \\
\hline
\end{tabular}


Annexe 2

\section{Modalités de formation et de suivis des stagiaires}

\begin{tabular}{|c|c|c|c|}
\hline Encadrement & Durée/nombre & $\mathrm{N}$ & Objectifs \\
\hline $\begin{array}{l}\text { Séminaires de formation } \\
\text { à la multimodalité et à } \\
\text { ses usages pédagogiques } \\
\text { et didactiques. }\end{array}$ & $\begin{array}{l}4 \text { séminaires (durée de } 3 \text { h chacun) } \\
\text { séminaires et rencontres compre- } \\
\text { nant des cours magistraux et des } \\
\text { rencontres individuelles offerts aux } \\
\text { stagiaires entre janvier et avril } 2013 .\end{array}$ & $N=16$ & $\begin{array}{l}\text { Discussion des projets possibles avec chaque stagiaire. } \\
\text { Présentation des attentes du projet d'innovation et des contraintes } \\
\text { s'y rattachant. } \\
\text { Recherche d'idées et de ressources. } \\
\text { Modalités d'intégration de la multimodalité aux contenus à ensei- } \\
\text { gner. } \\
\text { Détermination de situations problèmes et conceptualisation des } \\
\text { projets. } \\
\text { Présentation du cadre de la multimodalité et formation à ses } \\
\text { usages dans le champ de la littératie et de ressources techno- } \\
\text { logiques, numériques en lien avec des usages pédagogiques } \\
\text { disciplinaires. }\end{array}$ \\
\hline Rencontres Skype & 3 rencontres d'environ une heure. & $N=1$ & $\begin{array}{l}\text { Demande d'aide et d'orientation pour le projet d'innovation. } \\
\text { Partage de difficultés inhérentes au contexte du stage (gestion de } \\
\text { classe, gestion de soucis liés aux ressources humaines et maté- } \\
\text { rielles, motivation des élèves.) } \\
\text { Adéquation des objectifs d'enseignements apprentissage avec les } \\
\text { attendus du projet d'innovation. }\end{array}$ \\
\hline Échanges courriel & 24 messages & $N=5$ & $\begin{array}{l}\text { Seulement } 5 \text { stagiaires ont sollicité de l'aide ou des éclaircisse- } \\
\text { ments par courriels. } \\
\text { Vingt-et-un (21) courriels concernant les projets d'innovation per- } \\
\text { sonnels de certains étudiants et trois (3) courriels plus généraux de } \\
\text { recadrage et d'explicitation des documents d'accompagnements } \\
\text { fournis aux stagiaires. }\end{array}$ \\
\hline
\end{tabular}


Annexe 3

\section{PLAN DU RAPPORT D’ANALYSE DU PROJET D’INNOVATION PÉDAGOGIQUE}

L'analyse de l'expérimentation d'un projet d'innovation pédagogique comportera les quatre (4) sections suivantes, chacune comprenant des étapes d'un processus de résolution de problèmes par l'innovation pédagogique.

\section{Compréhension du problème}

- Identification d'une problématique (identification d'un écart entre une situation observée et une situation désirée) et précision du problème (énoncé général);

- Description du problème (ses manifestations et ses principaux aspects);

- Recherche et localisation d'informations permettant d'y apporter une solution (courte recension d'écrits récents portant sur le problème et sa solution : minimum 3 sources);

- Objectifs pédagogiques ou didactiques poursuivis par le stagiaire.

\section{Intervention}

- Planification du projet en fonction du problème identifié et des éléments découlant de la recension des écrits;

- $\quad$ Planification des moyens envisagés pour vérifier les effets de l'intervention;

- Mise à l'essai du projet d'innovation pédagogique (description du contexte et des conditions de cet essai).

\section{Évaluation}

- Présentation des conséquences de l'intervention (quelles sont-elles?);

- Interprétation des conséquences de l'intervention (quels sont les liens entre l'intervention et ses conséquences?).

\section{Conclusion}

- Retour sur les objectifs poursuivis;

- Objectivation (apprentissages faits ou compétences développées par le stagiaire);

- $\quad$ Prospectives (réinvestissement possible dans des situations analogues). 


\section{Pour viser à réaliser un rapport de qualité, veillez à}

- la précision de l'énoncé du problème;

- la pertinence des écrits recensés;

- la précision de la planification du projet;

- la cohérence de l'intervention par rapport au contexte et aux écrits recensés;

- la clarté de la narration de l'intervention;

- l'originalité et au caractère novateur du projet;

- la précision des effets observés;

- la pertinence du jugement porté sur l'atteinte des objectifs du projet;

- la rigueur de la réflexion sur ses apprentissages;

- la richesse des pistes de réinvestissement;

- la qualité de la présentation matérielle du travail. 
TITRE: DISPOSITIF DIDACTIQUE POUR DÉVELOPPER LA LECTURE LITTÉRAIRE AU DEUXIÈME CYCLE DU SECONDAIRE

Auteur(s): MARIE-Christine BeAudry, Ph.D., UniVersité du QuÉBeC À MonTRÉAL (UQAM)

PUBLICATION: LA RECHERCHE-ACTION ET LA RECHERCHE-DÉVELOPPEMENT AU SERVICE DE LA LITTÉRATIE.

PAGES: $97-127$

Directeurs: ISABelle Carignan, Marie-Christine BeAudry et FrançoIS LAROSE

ÉdiTEUR: LES ÉDITIONS DE L'UNIVERSITÉ DE SHERBROOKE, 2016.

ISBN: 978-2-7622-0354-7

URI: HTTP://HDL.HANDLE.NET/11143/8810

DOI: HTTP://DX.DOI.ORG/10.17118/11143/8810 


\title{
Dispositif didactique pour développer la lecture littéraire au deuxième cycle du secondaire
}

\author{
Marie-Christine Beaudry, Ph.D., Université du Québec à Montréal (UQAM)
}

Résumé : Cet article fait état d'une recherche s'inscrivant dans la foulée des travaux sur la didactique de la lecture littéraire, sur l'établissement d'un rapport vivant entre le sujet lecteur et le texte littéraire. Cette recherche, qui prend la forme d'une recherche-développement, vise à élaborer un dispositif didactique en trois volets (le questionnement réciproque, l'écrit de travail et l'écriture d'invention) susceptible de développer la lecture littéraire chez des élèves du secondaire. La première version du dispositif didactique a été évaluée par cinq experts, soit des enseignants de français et des didacticiens. L'analyse de contenu de leurs commentaires a permis de procéder à l'amélioration du dispositif didactique. L'analyse a également permis de relever que le développement de la lecture littéraire effectué par des outils validés par des experts et créés en adéquation avec les avancées de la recherche et les besoins des enseignants s'avère encore crucial et nécessaire, avant même de songer à une mise en œuvre en salle de classe.

Mots-clés : Recherche-développement, dispositif didactique, lecture littéraire, enseignement secondaire. 


\section{Problématique}

\section{La didactique de la lecture littéraire : maladroitement développée et maitrisée}

Depuis les premiers travaux menés sur la lecture littéraire (notamment par Eco, 1985; Iser, 1985; Jauss, 1978 et Picard, 1986) puis sur le sujet lecteur (Langlade, 2001; Rouxel, 1996), didacticiens et enseignants de français expérimentent diverses pratiques afin de développer la lecture littéraire chez les élèves, notamment chez ceux du secondaire, niveau auquel s'est intéressée cette recherche. Bien que Dufays déplore que " jusqu'à ce jour, l'interrogation à [propos de la lecture littéraire] s'est centrée davantage sur la mise au point de modèles théoriques, de programmes d'apprentissage ou d'outils didactiques que sur l'étude des pratiques réelles des enseignants et de leurs effets en termes de perceptions et d'apprentissages chez les élèves » (Dufays, 2006, p. 80), élaborer des outils pour soutenir adéquatement le développement de la lecture littéraire s'avère encore crucial. D'une part, la « didactisation » de la lecture littéraire requiert une réflexion éprouvée et validée, autant par les didacticiens que par les enseignants, sur les outils et les pratiques à privilégier pour placer l'élève au centre de l'acte de la lecture. Bien que plusieurs didacticiens proposent différentes pratiques afin d'accorder une plus grande place au lecteur et de le développer, telles que le cercle de lecture et le journal dialogué (Hébert, 2002; Lebrun, 1996), l'écrit de travail (Tauveron, 2002), l'écriture d'invention (Rouxel, 2007) ou encore le débat interprétatif (Poslaniec, 2002), l'approche formaliste pour développer la lecture littéraire semble encore présente au secondaire. En effet, Dezutter, Larivière, Bergeron et Morissette (2007) ont relevé la présence de pratiques dans les classes de français du secondaire au Québec qui laissent moins de place au lecteur; par exemple, le questionnaire de lecture visant à vérifier la compréhension et le résumé de lecture de l'œuvre demeurent deux pratiques relativement fréquentes. Contrairement, entre autres, aux travaux de Jauss (1978) et de Picard (1986) qui soulignent le rôle primordial joué par le lecteur, ces deux pratiques ne lui laissent que peu de place et ne favorisent pas nécessairement la prise de conscience, chez l'élève, de la nature évolutive de sa compréhension et de son interprétation (Langlade, 2007a).

De surcroit, les problèmes rencontrés par les élèves du secondaire en compréhension et en interprétation, qui entravent la lecture littéraire, témoignent de la nécessité de développer des dispositifs qui contribuent à leur développement comme lecteurs littéraires (Beaudry, 2009). En effet, il ressort de diverses études que la mise en œuvre de stratégies cognitives et métacognitives pour comprendre pose problème pour l'élève du secondaire (Fayol, 1992; MÉLS, 2005; Van Grunderbeeck, Théorêt, Cartier, Chouinard et Garon, 2004), telles qu'anticiper, synthétiser les informations du texte, établir des liens entre différentes parties du texte et discriminer les informations importantes. II semble également que le genre textuel présente des défis de compréhension; en effet, certains élèves peinent à effectuer une représentation mentale du texte (Van Grunderbeeck et coll., 2004) et pour le texte narratif, en saisir la logique et les modes de narration leur est difficile (Tauveron, 2001). Enfin, certains problèmes de compréhension sont liés à la méconnaissance du sens de certains mots, engendrant 
parfois une atomisation de la lecture (Montballin, 1999). Quant à l'interprétation, processus itératif et complémentaire à la compréhension, elle pose elle aussi des défis aux élèves. La nature même de l'interprétation présente des défis de taille pour les élèves. Premièrement, activité « négociée » (Vandendorpe, 1992) dans un rapport d'égalité entre les interlocuteurs, l'interprétation implique un échange entre le lecteur et le texte, et aussi entre la communauté des lecteurs, nommée parEco (1992) la « communauté d'interprétants ». L'interprétation est donc sujette à discussion et cette possibilité d'échanges, de mises en commun des interprétations entre les élèves, qui limite et garantit les interprétations possibles d'un texte, peut aussi faire craindre à certains élèves de s'exposer (Chabanne et Bucheton, 2002) et de présenter leur interprétation. Deuxièmement, certains problèmes des élèves découlent du foisonnement de leurs interprétations et de leur difficulté à établir des limites à leurs interprétations. Par exemple, certains adoptent une distance excessive face au texte lu (Rouxel, 1996) ou, au contraire, poussent trop loin l'illusion référentielle (Beaudry et Huneault, 2011) et voient dans le texte un sens qui ne s'y prête pas. Enfin, l'interprétation est alimentée par la culture, le bagage du lecteur; le lecteur «complète » le texte en puisant dans sa culture (Langlade, 2007a), ce qui lui permet de fonder son expérience de lecture, de confirmer à la fois sa compréhension et son interprétation. Si les caractéristiques socioculturelles du lecteur influent sur son interprétation et teintent sa vision du texte, elles peuvent également nuire à une interprétation lorsque l'ignorance de connaissances culturelles aboutit à une mésinterprétation, soit à une interprétation erronée (Beaudry et Huneault, 2011). Dans ces conditions, les élèves sont-ils véritablement outillés pour effectuer une lecture littéraire?

\section{Question et objectifs de la recherche}

Le développement de la lecture littéraire chez les élèves s'avère problématique. Selon Falardeau (2003), « bien des élèves du secondaire et du collégial n'ont pas acquis l'autonomie et les compétences qui leur permettraient de saisir et d'interpréter de manière créative des œuvres littéraires difficiles tout en restant fidèles au texte» (p. 675). La dimension interprétative de la lecture littéraire demeure maladroitement maitrisée par les élèves, en raison de l'absence ou d'une méconnaissance des stratégies pour effectuer une lecture littéraire complète. De même, certaines tâches données aux élèves pourraient biaiser, influencer, voire limiter la posture adoptée par les élèves (Beaudry et Huneault, 2011). Dans le cadre de cette recherche, nous nous sommes donc demandé si un dispositif didactique, qui travaillerait à la fois la compréhension et l'interprétation, peut contribuer à développer efficacement la lecture littéraire chez des élèves du deuxième cycle du secondaire (16-17 ans). Les objectifs poursuivis par cette recherche sont donc : 1) élaborer un dispositif didactique susceptible de faciliter le développement de la compréhension et de l'interprétation des élèves du deuxième cycle du secondaire à partir d'un roman contemporain québécois; 2) évaluer ce dispositif didactique; 3) améliorer le dispositif didactique créé. 


\section{Considérations théoriques}

\section{La lecture littéraire, un va-et-vient entre la compréhension et l'interprétation}

D’emblée, précisons qu'afin d'opérationnaliser une définition de la lecture littéraire, nous nous plaçons dans une position résolument didactique. La lecture littéraire résulte d'un va-et-vient, d'une interaction entre la compréhension et l'interprétation; le sens élaboré lors de la compréhension nourrit l'interprétation et vice-versa (Dufays, Gemenne et Ledur, 1996; Falardeau, 2003). Le lecteur y joue un rôle actif, créateur et libre : le texte littéraire n'existe totalement que par son intervention. Plus précisément, la compréhension entraine la saisie d'un sens déterminé et partagé par la communauté de lecteurs; on peut la définir, en quelque sorte, comme une soumission à des normes (Dufays, 1996; Jouve, 1993). Il serait cependant réducteur de limiter la compréhension à la reconnaissance d'un sens déjà présent, totalement inscrit dans le texte (Gervais, 2006); il revient à chaque lecteur de reconstruire le sens à partir de ses connaissances antérieures, de sa culture, de son bagage de lecteur. Le lecteur expert peut adopter diverses stratégies pour comprendre, telles qu'identifier les idées principales par rapport aux idées secondaires, résumer, repérer la structure du texte, réparer les pertes de compréhension, établir des liens avec ses connaissances antérieures. Quant à l'interprétation, elle est associée à un « regard critique, distancié » (Pamfil, 2007). Le lecteur interprète, «négocie » le sens du texte, tout en restant conscient que plusieurs interprétations demeurent possibles. Comme le théorise Eco (1992), tout lecteur qui interprète peut reconnaitre plusieurs sens, qui relèvent d'intentions distinctes: auctoris (le lecteur recherche des intentions conscientes et voulues de l'auteur), lectoris (le lecteur recherche ses propres intentions, le ou les sens qu'il a projetés au début ou en cours de lecture) et operis (le lecteur recherche des significations potentielles immanentes de l'œuvre). Différentes stratégies peuvent être mises en œuvre par le lecteur expert pour interpréter: décrire son expérience de lecture, ressentir de l'empathie pour les personnages ou s'identifier à l'un deux, imaginer d'autres mondes, soutenir son raisonnement à l'aide d'éléments du texte, d'éléments issus du contexte, de l'intertextualité, du paratexte, émettre des hypothèses sur la signification d'un élément du récit ou sur le récit en son ensemble, revoir et considérer l'évolution de son point de vue, etc. Si, tout comme pour la compréhension, l'interprétation se fonde sur des stratégies, celle-ci se base également sur les connaissances, les expériences, les sentiments, les résonnances que le lecteur établit entre le texte, son bagage de connaissances et d'expériences; ''investissement subjectif du lecteur est donc sollicité, voire requis par l'interprétation (Laglande, 2007a). 


\section{La lecture littéraire, un va-et-vient entre la participation et la distanciation}

Alternance entre une lecture réglée (game) et une lecture libre (playing), c'est-à-dire entre l'aspect rationnel de la lecture et son aspect passionnel, la double dimension de la lecture littéraire permet au lecteur d'accéder au sens du texte (Dufays, 1996; Picard, 1986). Le playing renvoie aux jeux de rôle, aux identifications à un personnage, tandis que le game désigne les stratégies employées par le lecteur (Rouxel, 2002). On constate donc un dédoublement du joueur, du lecteur, en deux sujets : le «lu » et le « lectant $»^{1}$ (Picard, 1986). Le « lu » serait du côté de l'abandon du lecteur, des identifications, du plaisir ressenti en cours de lecture, tandis que le « lectant», quant à lui, concerne la réflexion, la mise en œuvre critique d'un savoir, la mise à distance du lecteur avec ce qu'il lit (Picard, 1986). Ainsi, le lecteur oscille entre une lecture participante et une lecture de distanciation (Dufays, 1996). Dans la participation, il s'identifie au texte et s'intéresse au récit (lu). Par contre, en se distanciant du texte littéraire, il adopte une attitude critique face à ce dernier grâce à ses connaissances du champ littéraire, du fonctionnement de la langue et du texte (il est alors sujet lectant). La distanciation impose un écart entre le réel et la représentation; la distanciation se compare à un certain recul qui est la condition de la réception critique. Le lecteur se trouve alors en mesure d'expliciter et d'interpréter le texte littéraire, mais aussi de faire des liens entre les textes, de les comparer et de reconnaitre l'innovation (Dufays, 1996; Falardeau, 2003). Le lecteur peutégalement établir des relations entre le texte et son vécu, passé ou présent. Sa lecture « interpelle » son expérience du monde. Il se positionne alors comme un lectant, c'est-à-dire un lecteur actif et créateur qui apprécie esthétiquement la richesse du propos, la beauté formelle à partir des œuvres (Picard, 1986; Jouve 1993).

\section{Les principes pour la didactique de la lecture littéraire}

La lecture littéraire est « un équilibre particulier et à chaque fois renégocié entre ses diverses composantes » (Gervais et Bouvet, 2007, p. 1); pour qu'elle advienne, la compréhension et l'interprétation doivent toutes deux être sollicitées. De même, les pratiques didactiques mises en place doivent contribuer le plus possible à ce que l'apprenant se perçoive comme un lecteur, autorisé à faire preuve de subjectivité et à convoquer ses expériences (Langlade, 2007a). D’abord, cela signifie, pour la didactique de la lecture littéraire, de privilégier des activités développant la compréhension de pair avec des activités requérant une interprétation (Dufays, 1997). Pour l'enseignant, cela implique qu'il accorde une importance à l'acquisition et à la maitrise des savoirs nécessaires à la saisie des caractéristiques formelles d'un texte, tout autant qu'à l'acquisition des compétences qui permettent l'élaboration, l'activation et l'analyse des stratégies de lecture (Langlade, 2001) qui servent à comprendre et à interpréter. Quant aux activités développant l'interprétation, elles doivent favoriser l'émergence des interprétations des élèves et permettre les essais et erreurs, les égarements interprétatifs; mais «si, avec des enfants de 7-8 ans, la dynamique de groupe et l'absence d'inhibition permettent des

1. Picard (1986) reconnait trois sujets lecteurs, le troisième étant le liseur. Celui-ci serait du côté du réel, du côté de la lecture à proprement parler, du lecteur qui lit, qui sent le poids du livre. 
échanges plus ouverts à propos des textes, les préjugés défavorables des adolescents et leur crainte du jugement de l'autre impliquent une participation et une animation plus actives du maitre dans le travail d'appropriation des textes par les élèves » (Falardeau, 2003, p. 676). II convient alors de privilégier des activités lors desquelles l'enseignant joue davantage le rôle d'un guide, d'un facilitateur des interprétations (Tauveron, 2002), en concomitance avec d'autres activités qui lui permettent d'expliciter des stratégies pour interpréter. Le rôle de l'enseignant se trouve alors décentré; de détenteur de la «bonne réponse », de la bonne interprétation, il devient un guide et modèle pour réaliser une lecture littéraire complète. Son interprétation ne doit donc pas être complètement dévoilée; au contraire, celle-ci doit demeurer «masquée» (Falardeau, 2003) afin de ne pas interférer dans le travail interprétatif des élèves en teintant leurs réponses. Conséquemment, favoriser les interactions entre les élèves, et entre les élèves et l'enseignant, constitue un principe primordial pour développer la lecture littéraire; en effet, « la créativité interprétative est fortement stimulée par des émotions collectives, des expériences partagées, les échanges dans la classe, les travaux de groupes » (Bucheton, Brénas, Chabanne et Dupuy, 2004, p. 13). Les interactions orales jouent alors un rôle médiateur essentiel dans la construction de l'interprétation. En outre, un élève s'autorisera davantage à explorer diverses interprétations quand le groupe, collectivement, fait également part de ses émotions et de ses interprétations (Bucheton et coll., 2004).

Enfin, la didactique de la lecture littéraire, comme tout autre apprentissage, doit s'ancrer dans les expériences de l'élève et dans ses connaissances culturelles, qui lui serviront de première référence pour sa compréhension et son interprétation (Falardeau, 2003). Sans quoi seuls « les héritiers 》 (Bourdieu et Passeron, 1985), les élèves ayant été dès leur plus jeune âge en contact avec la culture dite "savante ", seraient en mesure de comprendre et de construire une interprétation cohérente du texte littéraire. Le défi de l'enseignant consiste à guider l'élève pour qu'il passe du lieu réconfortant que constitue sa culture personnelle à une culture inconnue, nouvelle (Zakhartchouk, 1999). Pour ce faire, la sélection de romans, classiques ou contemporains, qui comportent des sentiments, des préoccupations et des gestes quotidiens qui rejoignent ceux des élèves (Zakhartchouk, 1999), constitue un moyen pour faciliter l'entrée dans la lecture littéraire des élèves. La mise en place d'activités « inaugurales » et « augurales », qui permettent de présenter l'œuvre à lire tout en favorisant l'activité interprétative (Langlade, 2002), représente aussi un autre moyen pour « amorcer l'ancrage » de la lecture littéraire dans les expériences de l'élève et contribue à ce qu'ils vivent une véritable expérience de lecture.

En fonction de ces principes pour la didactique de la lecture littéraire, il est possible de dégager des pratiques favorisant le développement de la lecture littéraire, telles que le cercle de lecture, le journal dialogué, le questionnement réciproque, l'écriture d'invention et les écrits de travail. Le cercle de lecture et le journal dialogué ayant fait l'objet de plusieurs recherches (Hébert, 2002; Lebrun, 1996; Terwagne, 2003), il sera ici question des pratiques du questionnement réciproque, des écrits de travail et de l'écriture d'invention. 
Aussi nommé Reciprocal Questioning (ReQuest) par Manzo (1969), le questionnement réciproque consiste à découper le texte en segments significatifs et, après chaque lecture, à questionner (à l'oral comme à l'écrit) les élèves en grand groupe ou en petits groupes. Qu'elles viennent de l'enseignant ou des élèves, les questions peuvent s'adresser aux pairs et à l'enseignant; les élèves révèlent leur compréhension du texte autant par leurs réponses que par leurs questions (Lebrun et Le Pailleur, 1992). Dumortier (1991) suggère même de lier « le questionnement aux objectifs et aux apprenants, pas aux textes. Ces derniers, en effet, n'imposent aucune question... et les permettent toutes » (p. 74). Un tel positionnement favoriserait l'expression des réactions des élèves, contribuerait à résoudre collectivement une incompréhension ainsi qu'à élaborer et à échanger les interprétations (Tauveron, 2002). En outre, un questionnement varié peut susciter des réponses autant créatives qu'axiologiques; il favorise alors l'engagement du lecteur (Langlade, 2007b) en permettant d'interroger le « texte de l'élève », la version personnelle et achevée du texte littéraire par l'élève. Les questions peuvent donc contribuer à faire passer les élèves d'une posture participative, d'identification au texte à une posture de «lectant» (Picard, 1986), c'est-à-dire de distanciation critique à l'égard de celui-ci. Il importe toutefois d'éviter que les questions ne vérifient qu'une compréhension de surface : un dispositif de questionnement est fertile dans la mesure où les questions ouvrent un espace d'échanges et d'argumentation (Dumortier, 1991; Langlade, 2007a). Il semblerait en effet que certaines questions favorisent peu la compréhension de l'élève puisqu'elles visent surtout à vérifier s'il a lu; par conséquent, elles l'incitent bien souvent à retenir des éléments non pertinents pour la compréhension globale du texte. Enfin, la discussion suscitée par les questions décentre l'élève et développe son habileté à prendre en compte le savoir et les opinions des autres (Lebrun et Le Pailleur, 1992). Quant à l'enseignant, il lui revient d'apporter des rectifications ou des précisions non seulement sur les réponses élaborées, mais aussi sur les stratégies employées.

\section{Les écrits de travail}

Élaborée d'abord pour la didactique des sciences, la pratique des écrits de travail se révèle fort utile en lecture en raison de la variété des écrits que l'apprenant peut rédiger et de la possibilité de travailler la compréhension et l'interprétation. Ces textes dits « intermédiaires » favorisent l'établissement de liens entre le texte lu et le lecteur, entre une première lecture et une relecture. Ils consistent à faire produire des textes, avant, pendant et après la lecture, dans lesquels l'élève note sa pensée, lui donne une forme et met en relation ses idées (Tauveron, 2002), émet un jugement et développe sa réflexivité (Houdart-Merot, 2004; Huyhn, 2004). Cette pratique n'est cependant pas sans difficulté pour l'élève du secondaire : l'analyse de commentaires littéraires de lycéens montre que souvent, la subjectivité du lecteur est absente (De Beaudrap et Benoit, 1999). En outre, de nombreuses copies présentent des difficultés au niveau de l'argumentation : la progression des idées est plus ou moins présente, les exemples tirés du texte ne figurent pas toujours et, quand ils le sont, leur pertinence pose problème. Comme le notent De Beaudrap et Benoit (1999), « la volonté de démontrer quelque chose s'affiche [...], mais elle ne permet pas de faire naitre une véritable interprétation du texte » (p. 
127). Les écrits de travail demandent donc entrainement, accompagnement et modélisation de la part de l'enseignant afin qu'ils contribuent à développer la lecture littéraire. Le tableau 1 synthétise les différents écrits de travail que peut produire un élève.

Tableau 1 - Typologie des écrits de travail (Houdart-Merot, 2004; Huyhn, 2004; Tauveron, 2002)

\begin{tabular}{|c|c|c|}
\hline Types d'écrits & Fonction & Apports pour l'élève et l'enseignant \\
\hline \multicolumn{3}{|l|}{ Avant la lecture } \\
\hline $\begin{array}{l}\text { Exprimer ses premières impressions de } \\
\text { lecture à partir de l'incipit }\end{array}$ & $\begin{array}{l}\text { Exprimer les premières impressions de } \\
\text { lecture }\end{array}$ & $\begin{array}{l}\text { Identification des erreurs de compréhension qui peuvent } \\
\text { influencer la lecture }\end{array}$ \\
\hline \multicolumn{3}{|l|}{ Pendant la lecture } \\
\hline $\begin{array}{l}\text { Rédiger des questions qui permettent de } \\
\text { comprendre le texte }\end{array}$ & $\begin{array}{l}\text { Problématiser la lecture et identifier les } \\
\text { éléments essentiels du texte }\end{array}$ & Résolution des incompréhensions \\
\hline $\begin{array}{l}\text { Reformuler le texte lu pour ensuite discu- } \\
\text { ter de la reformulation }\end{array}$ & $\begin{array}{l}\text { Reformuler et confronter des interpréta- } \\
\text { tions individuelles }\end{array}$ & $\begin{array}{l}\text { - Identification des erreurs de compréhension et d'inter- } \\
\text { prétation } \\
\text { - Enrichissement, par les échanges, des interprétations }\end{array}$ \\
\hline $\begin{array}{l}\text { Écrire, tout au long de la lecture, ses } \\
\text { impressions et ses hypothèses de lecture }\end{array}$ & $\begin{array}{l}\text { Témoigner de l'évolution de la compré- } \\
\text { hension et de l'interprétation }\end{array}$ & $\begin{array}{l}\text { - Réflexion métacognitive sur la pratique de lecture } \\
\text { - Affinage de la compréhension }\end{array}$ \\
\hline \multicolumn{3}{|l|}{ Après la lecture } \\
\hline $\begin{array}{l}\text { Exprimer ses ultimes impressions de } \\
\text { lecture }\end{array}$ & $\begin{array}{l}\text { Exprimer les ultimes impressions de } \\
\text { lecture }\end{array}$ & $\begin{array}{l}\text { - Identification des erreurs de compréhension et d'inter- } \\
\text { prétation } \\
\text { - Trace de l'interprétation }\end{array}$ \\
\hline $\begin{array}{l}\text { Réécrire l'histoire lue en changeant de } \\
\text { point de vue }\end{array}$ & $\begin{array}{l}\text { Témoigner de la compréhension du } \\
\text { texte lu }\end{array}$ & Identification des incompréhensions \\
\hline $\begin{array}{l}\text { Schématiser l'interprétation en mettant } \\
\text { en évidence certains éléments du texte }\end{array}$ & $\begin{array}{l}\text { Présenter visuellement l'interprétation et } \\
\text { hiérarchiser certains éléments du texte }\end{array}$ & Légitimation d'une ou des interprétations \\
\hline $\begin{array}{l}\text { S'exprimer sur le texte lu, sans le relire, à } \\
\text { partir des souvenirs gardés }\end{array}$ & Exprimer le souvenir de lecture & $\begin{array}{l}\text { - Appropriation personnelle du texte } \\
\text { - Consolidation de l'expérience de lecture }\end{array}$ \\
\hline $\begin{array}{l}\text { Rédiger un commentaire critique sur une } \\
\text { stratégie de lecture employée, sur un } \\
\text { autre écrit de travail, sur une rédaction } \\
\text { créative }\end{array}$ & $\begin{array}{l}\text { Justifier et critiquer des choix, établir des } \\
\text { liens, souligner ce qui a facilité ou fait } \\
\text { obstacle }\end{array}$ & $\begin{array}{l}\text { - Explicitation de la pensée, des hypothèses, des inter- } \\
\text { rogations } \\
\text { - Détection des erreurs de compréhension, des mésin- } \\
\text { terprétations }\end{array}$ \\
\hline \multicolumn{3}{|l|}{ À tout moment } \\
\hline $\begin{array}{l}\text { Rédiger un texte narratif à partir de } \\
\text { tâches partielles d'écriture }\end{array}$ & $\begin{array}{l}\text { Travailler la compréhension de l'univers } \\
\text { lu }\end{array}$ & $\begin{array}{l}\text { Compréhension du processus d'écriture et des caractéris- } \\
\text { tiques d'un genre littéraire }\end{array}$ \\
\hline $\begin{array}{l}\text { Réagir à des passages sélectionnés pour } \\
\text { ensuite confronter son interprétation }\end{array}$ & $\begin{array}{l}\text { Provoquer des interprétations diver- } \\
\text { gentes sur des passages problématiques }\end{array}$ & Découverte de la pluralité des interprétations \\
\hline
\end{tabular}




\section{L'écriture d'invention}

Lors de la pratique de l'écriture d'invention, l'élève s'inspire d'un texte littéraire ou d'un corpus de textes littéraires pour le pasticher, le transformer, le poursuivre (Houdart-Merot, 2004; Huyhn, 2004). La pratique de l'écriture d'invention vise, entre autres, à proposer une alternative au formalisme avec lequel sont souvent abordés et discutés les textes, au rapport parfois sacralisé entretenu envers le texte littéraire (Daunay et Denizot, 2003). Activité complexe et créative, l'écriture d'invention convoque à la fois le lecteur et le scripteur. Dans un premier temps, l'élève lit le texte littéraire et peut ensuite en analyser la structure; dans un second temps, il devient scripteur et procède à la rédaction de son texte d'invention. Quoique l'élève adopte une posture distante afin d'analyser les propriétés du texte (entre autres narratives et thématiques), l'écriture d'invention convoquerait davantage une posture participative : lors de la relecture, ce serait d'abord cette dernière qui serait interpelée, moins pour analyser les propriétés du texte «que pour en exploiter et en explorer les possibilités» (Petitjean, 2002, p. 16). Bien que la tâche d'écriture, notamment lorsque l'élève réalise un pastiche ou tente de se rapprocher le plus possible de l'écriture de l'auteur, teinte la lecture littéraire de l'élève ainsi que son texte d'invention (Beaudry et Huneault, 2011; Le Goff, 2004), l'analyse et l'écriture lui permettent de montrer sa compréhension et son interprétation du texte lu, mais aussi sa compétence à écrire des textes littéraires.

\section{Modèle d'action}

Qu'on l'appelle « plan pédagogique » (Lebrun et Berthelot, 1994), « désign pédagogique » (Stolovitch et LaRocque, 1983) ou « recherche-développement » (Van der Maren, 1996), ce type de recherche s'avère indispensable pour développer un système d'enseignement et d'apprentissage à la fois rigoureux et économique en termes de cout et de temps. Recherche qui met «à l'épreuve la théorie » (Guichon, 2007, p. 43), la recherche-développement crée le lien entre les théories de l'enseignement, celles de l'apprentissage et les pratiques : l'outil ou le dispositif didactique développé tient compte de tous ces éléments (Loiselle, 2001). Comme le souligne Van der Maren (1996), par la recherche-développement, le chercheur analyse «les applications pratiques possibles d'une idée [...] pour mettre au point un produit » (p. 64). On ne peut que déplorer que, malgré sa pertinence certaine, la recherche-développement « occupe une très petite part de la recherche actuelle en éducation » (Loiselle et Harvey, 2007, p. 41).

Les objectifs centraux de cette recherche-développement concernaient la création, l'évaluation et l'amélioration d'un dispositif didactique. La méthodologie adoptée pour concevoir le prototype s'inspire des étapes proposées par Van der Maren (1996) ainsi que par Lebrun et Berthelot (1994): 1) la définition du problème, l'identification des besoins et l'élaboration des objectifs didactiques poursuivis par la création du dispositif; 2) la conception du prototype; 3) son évaluation; et, enfin, 4) son amélioration pour en arriver au dispositif didactique final. Nous présentons brièvement ici les 
grandes lignes des deux premières étapes; l'évaluation et l'amélioration du prototype font l'objet de sections distinctes dans cet article.

\section{Définition du problème, identification des besoins et élaboration des objectifs didactiques poursuivis par le dispositif}

La recension des écrits a permis de soulever différentes dimensions au problème de la didactique de la lecture littéraire. De cette recension ressort nettement une préoccupation chez les didacticiens à proposer des pratiques et des outils qui favorisent une posture de lecture littéraire chez les élèves, de même qu'une difficulté pour les élèves du secondaire à réellement effectuer une lecture littéraire. Développer un outil qui contribue au développement de la lecture littéraire s'avère ainsi nécessaire. Le dispositif vise donc à amener les élèves à comprendre un texte littéraire et à l'interpréter. Le dispositif didactique s'appuie sur un roman contemporain québécois.

\section{Conception du prototype}

Pour concevoir le prototype, les objectifs didactiques spécifiques de ce dernier (amener les élèves à comprendre et à interpréter) ont été étroitement liés au cadre théorique, ce qui a amené la sélection de pratiques didactiques adéquates au développement de la lecture littéraire, selon différentes recherches théoriques et empiriques (telles que celles de Dufays, 1996; Falardeau, 2003; Hébert, 2002; Langlade, 2001;2007a; Lebrun, 1996; Rouxel, 1996; 2007 et Rosenblatt, 1995, pour ne mentionner que celles-ci). Trois critères ont été utilisés pour sélectionner les pratiques : celles-ci devaient travailler la compréhension et l'interprétation, tout en favorisant la liberté interprétative des élèves (principes didactiques). En outre, les pratiques choisies devaient représenter un certain défi cognitif pour des élèves de la fin du secondaire. Voilà pourquoi le questionnement réciproque, les écrits de travail et l'écriture d'invention ont été retenus. Enfin, ces pratiques ont moins fait l'objet de recherches que les pratiques du cercle de lecture et du journal dialogué. De nombreux essais et erreurs ont été effectués et nous ne saurions négliger l'apport de notre double rôle de chercheur et de praticien dans la conception du prototype soumis aux experts, dont la première version est présentée dans la section suivante.

\section{Justification du choix du roman}

Au Québec, il n'existe aucun corpus obligatoire ni de consensus sur les œuvres à enseigner, même si le ministère de l'Éducation recommande la lecture d'œuvres québécoises et qu'il propose quelques principes pour les exploiter. Il revient aux institutions scolaires, du primaire à l'université, de proposer un corpus d'œuvres. Nikolski, de Nicolas Dickner (2007), a été choisi pour la conception du prototype pour plusieurs raisons. Premièrement, la complexité de ce roman permet de développer la lecture littéraire : cette complexité relève entre autres des traits spécifiques du roman (trois points de vue de narration, cinq personnages importants et tous liés, différentes époques, lieux divers...) et 
de la richesse des expériences de lecture qu'il peut générer (de nombreux blancs sont à combler, la quête de chacun des personnages peut interpeler le lecteur). II revient au lecteur, hors de tout doute, d'achever Nikolski. Ce roman permet donc de développer la compréhension (faire des inférences est nécessaire à de nombreuses reprises) et l'interprétation (par exemple, la fin ouverte et les trois histoires n'offrent pas beaucoup de réponses au lecteur). D'ailleurs, il semblerait que le développement de stratégies qui aident à la compréhension serait davantage favorisé lorsque les apprenants sont en contact avec des textes plus difficiles (Falardeau, 2003). L'aspect socioculturel constitue un autre critère qui justifie que Nikolski ait été retenu : nous souhaitions travailler une œuvre valorisée par l'institution culturelle et qui sert de référence dans la société (Dufays, Gemenne et Ledur, 1996), mais qui peut susciter des discussions sur sa valeur littéraire. Soulignons que Nikolski a été cinq fois récompensé : il a reçu le Prix des libraires du Québec $15^{e}$ anniversaire (2008), le Prix des libraires du Québec (2006), le Prix des collégiens (2006), le Prix Anne-Hébert (2006) ainsi que le Prix Printemps des lecteurs-Lavinal (2007). Choisir un roman contemporain, de surcroit le premier roman d'un jeune auteur, élargit le champ littéraire dans lequel s'effectue l'enseignement de la lecture littéraire. Par les activités mises en place en classe, il devient alors possible d'engager l'élève dans le processus de « littérarisation » d'un roman contemporain (Privat, 2005) et même de remettre en question cette valeur littéraire. Cela peut contribuer à rendre l'élève attentif à la production romanesque actuelle, et à ainsi le sensibiliser à la culture (aux productions culturelles, aux pratiques culturelles) dans laquelle il évolue. Troisièmement, nous souhaitions accorder la priorité aux titres québécois, notamment à la littérature contemporaine québécoise (Thérien, 1997). Enfin, mentionnons qu'un roman contemporain offre l'avantage indéniable, pour un enseignant de français, de n'être précédé que de quelques commentaires et analyses.

\section{Prototype}

Dans la version intégrale du prototype soumise à l'évaluation par des experts, la durée, la pratique employée, la composante de la lecture littéraire sollicitée (compréhension, interprétation), la notion à l'étude, le type de connaissances ainsi que les documents à remettre à l'élève et ceux utiles à l'enseignant étaient précisés et fournis pour chacun des 11 cours (75 minutes chacun). Nous présentions également les tâches réalisées par l'enseignant et celles des élèves. Toutes les activités proposées sont organisées autour du roman Nikolski. Les grandes lignes constituant le prototype soumis aux experts sont présentées dans le tableau 2. 
Tableau 2 - Résumé du prototype

COURS 1
Entrée dans la lecture littéraire de Nikolski et présentation des tâches à réaliser

Composante de la lecture littéraire

Compréhension

Interprétation

\section{Pratique didactique}

Questionnement réciproque

\section{Résumé du cours}

1. Anticipation, puis entrée dans la lecture du roman

1.1 En grand groupe, l'enseignant questionne les élèves sur le potentiel significatif des titres de films et de romans: Titanic, Harry Potter et la chambre des sorciers, Le Diner de cons, Star Wars, Bon cop bad cop, The Godfather, C.R.A.Z.Y, Quatre filles et un jeans, Les misérables, Le parfum... Ces titres sont-ils représentatifs du contenu du film ou du roman? Nous donnent-ils une bonne idée du récit? Nous aident-ils à comprendre l'histoire racontée?

1.2 Lecture individuelle du titre du roman, de l'incipit et de la table des matières (présente dans ce roman) et anticipation de la part des élèves, d'abord réalisée de manière individuelle dans un carnet de notes.

1.3 L'enseignant demande aux élèves de partager collectivement leur anticipation du récit et leurs premières impressions.

2. Présentation des tâches et échéances

2.1 L'enseignant présente l'auteur aux élèves, leur fournit des reproductions des cartes géographiques du Canada, du Québec, de Montréal et de l'Alaska, cartes nécessaires à la compréhension du roman, puis présente les grandes lignes du travail à réaliser et les échéances : différents écrits, questions et discussions en équipe et en grand groupe qui seront évalués de manière formative; des commentaires critiques et une rédaction créative inspirée du roman qui feront l'objet d'une évaluation sommative. On peut compter une semaine et demie pour la lecture du roman.

2.2 L'enseignant présente le travail spécifique qui doit être fait pendant la lecture du roman : les élèves sont regroupés en équipe et chacune se voit attribuer des questions de compréhension par rapport au roman. Tout au long de la lecture du roman, chaque membre d'une équipe doit répondre aux questions attribuées. Les notes de lecture sont à consigner dans un carnet de lecture. Les élèves doivent préparer des mini-leçons, c'est-à-dire qu'ils devront expliquer aux autres élèves de la classe le contenu dont ils étaient responsables (cours 3 et 4).

\section{COURS 2}

Étude de Nikolski - une fois que le roman est lu

\section{Composante de la lecture littéraire}

Compréhension

Interprétation

\section{Pratique didactique}

Questionnement réciproque

\section{Résumé du cours}

L'enseignant rappelle le travail : les élèves doivent mettre en commun leurs réponses et poursuivre le travail. L'enseignant circule d'équipe en équipe et aide à parfaire leur compréhension et leur interprétation.

\section{COURS 3 ET COURS 4}

Mise en commun du travail d'étude de Nikolski

\section{Composante de la lecture littéraire}

Compréhension

Interprétation

\section{Pratique didactique}

Questionnement réciproque

\section{Résumé du cours}

L'enseignant invite les élèves à présenter à la classe les réponses aux questions auxquelles chaque équipe devait répondre. L'enseignant en profite pour rectifier et soulever d'autres questions. 
COURS 5

Réflexion sur le processus de littérarisation d'une œuvre

Composante de la lecture littéraire

Interprétation

\section{Pratique didactique}

Questionnement réciproque

Écrits de travail

\section{Résumé du cours}

1. L'enseignant demande aux élèves de se regrouper en équipe de 4 et leur demande de répondre aux questions suivantes : 1) Qu'est-ce qui confère à un texte sa valeur « littéraire »? 2) Qu'est-ce que la littérature?

2. Collectivement, les élèves mettent en commun les critères trouvés. Il est important de leur faire découvrir qu'il existe un flou, une absence de critères précis et stables.

3. L'enseignant rappelle aux élèves les cinq prix reçus par le roman: Prix des libraires du Québec 15e anniversaire (2008), Prix des libraires du Québec (2006), le Prix des collégiens (2006), le Prix Anne-Hébert (2006) ainsi que le Prix Printemps des lecteurs-Lavinal (2007). Ensuite, il distribue les documents "Quelques informations sur les prix littéraires », «Quelques critiques de Nikolski » et «Entrevue avec Nicolas Dickner ». Après avoir lu individuellement les trois documents, l'enseignant demande aux élèves de poursuivre leur réflexion, individuellement, en répondant aux questions suivantes: Quels sont les éléments du roman qui, d’après vous, légitiment de donner un ou des prix littéraires à Nikolski? Le roman contient-il, au contraire, des éléments qui ne légitiment pas de lui donner des prix? Donnez des arguments précis appuyés par des exemples, des citations tirées du roman. Votre point de vue peut être partagé. Les élèves auront à rédiger un écrit de travail lors du prochain cours sur ces questions spécifiques et ce texte fera l'objet d'une évaluation sommative.

\section{COURS 6}

Réflexion sur le processus de littérarisation d'une œuvre

\section{Composante de la lecture littéraire}

Interprétation

\section{Pratique didactique}

Écrits de travail

\section{Résumé du cours}

1. En équipe de 4, les élèves échangent leur point de vue quant aux questions travaillées lors du cours 5.

2. À la lumière de la discussion, les élèves rédigent un bref commentaire critique.

\section{COURS 7}

Début de la rédaction créative

\section{Composante de la lecture littéraire}

Compréhension

Interprétation

\section{Pratique didactique}

Questionnement réciproque

Écriture d'invention

\section{Résumé du cours}

1. L'enseignant présente aux élèves la tâche à réaliser : écrire une rédaction créative inspirée du roman : À la manière de Nicolas Dickner, choisissez l'un des personnages suivants et imaginez son parcours, en situant l'action dans une ou des divisions du roman (1989, 1990, 1994, 1995, 1999), comme si vous ajoutiez un chapitre. Vous devez insérer au moins une allusion culturelle et développer votre récit autour d'un des thèmes abordés. Minimum 250 mots.

- Sarah: ce qu'elle fait pendant l'absence de son fils, les endroits où elle passe, son parcours géographique et émotif.

- Jonas Doucet: ce qu'il a fait sur l'ile Nikolski, comment il s'y est rendu, son parcours géographique et émotif.

Cette rédaction fera l'objet d'une évaluation sommative.

2. Individuellement, les élèves notent quelques idées dans leur carnet, puis se regroupent en équipe de 4 élèves afin d'effectuer un remue-méninge, de valider et de rectifier leurs idées.

3. L'enseignant invite les élèves à commencer, individuellement, le plan et le brouillon de leur rédaction. 


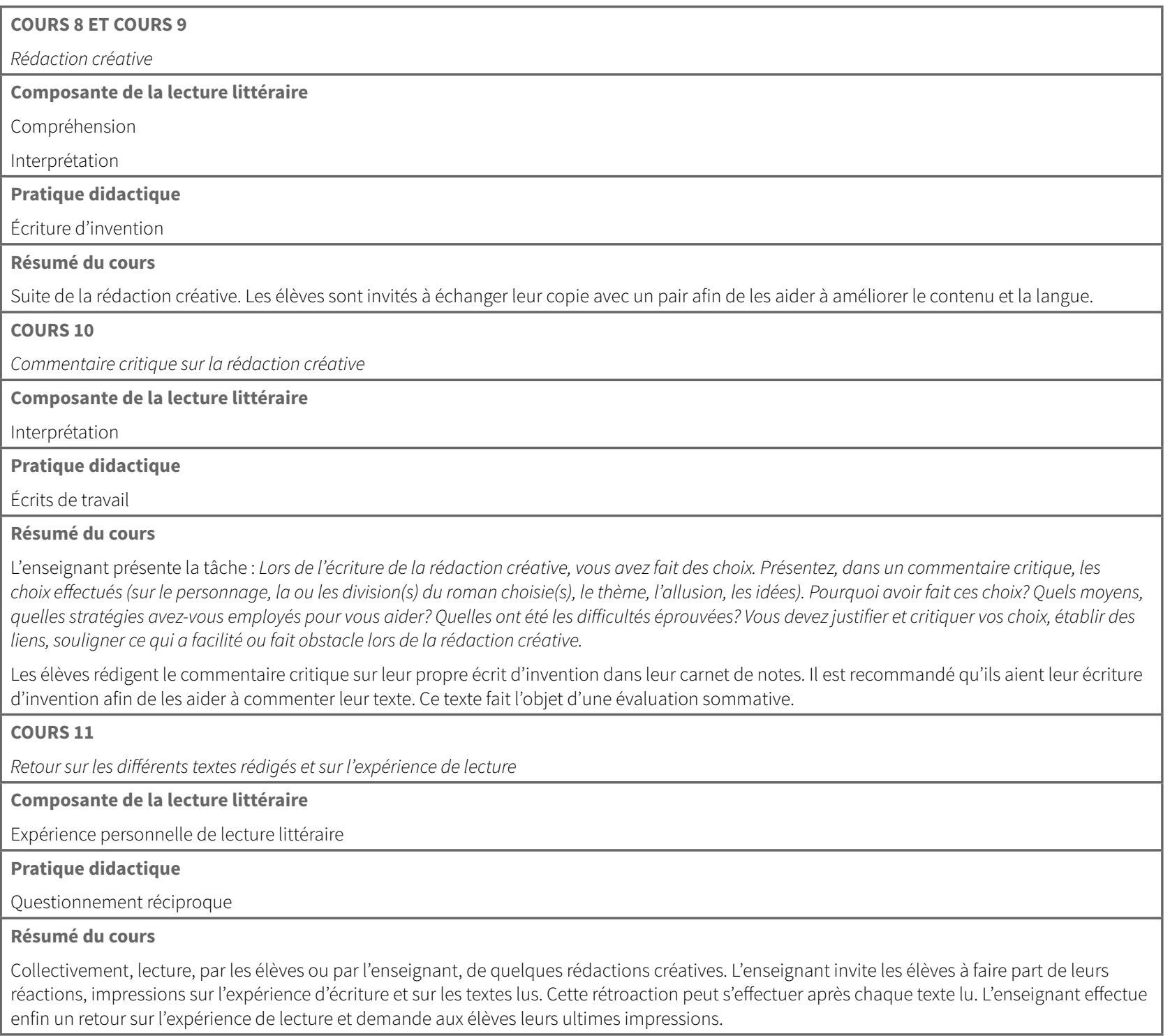

\section{Mise à l'essai du prototype avec des experts}

L'outil développé dans le cadre d'une recherche-développement peut faire l'objet d'une évaluation par des experts, puis d'une mise à l'essai formelle en classe, ou alors uniquement d'une évaluation, aussi nommée mise à l'essai avec des experts. Lors de l'évaluation de cet outil, « la constitution d'une équipe regroupant le chercheur-développeur et des gens du milieu parait souhaitable afin d'assurer une meilleure adéquation entre le produit développé et les besoins du milieu » (Loiselle et Harvey, 2007, p. 52) et nous ajoutons avec la réalité du « terrain », de la pratique; voilà pourquoi cinq experts du milieu de l'enseignement ont été sollicités. Cette évaluation permet, entre autres, d'évaluer le cadre théorique qui sous-tend les choix effectués (Loiselle, 2001), de vérifier l'adéquation du dispositif avec les résultats les plus récents de la recherche théorique (Loiselle et Harvey, 2007) et, enfin, d'identifier les améliorations à apporter au dispositif avant de songer à une mise à l'essai auprès 
d'élèves du secondaire. Cette évaluation par plusieurs experts permet d'obtenir une précieuse rétroaction, relativement objective, afin d'améliorer le dispositif.

Deux catégories d'experts ont été sollicitées afin de procéder à l'évaluation du prototype. Dans la première catégorie, deux enseignantes ont évalué le prototype. La première enseigne le français en cinquième secondaire depuis plus de 12 ans, en milieu multiethnique, dans une école de Montréal. La seconde enseigne elle aussi le français en cinquième secondaire, depuis plus de 25 ans, dans une école en Abitibi-Témiscamingue. II nous importait de recevoir des commentaires de deux enseignantes qui évoluent dans un milieu différent: ainsi, dans le but de permettre une relative généralisation et de répondre le plus possible aux besoins du plus grand nombre d'élèves et d'enseignants, nous avons amélioré notre dispositif didactique. Dans la deuxième catégorie d'experts, trois didacticiens-professeurs ont évalué notre prototype. Les deux premiers didacticiens sont spécialistes de la lecture, l'un respectivement en Belgique et l'autre en France. Quant au troisième expert, il est chercheur en didactique du français en France et s'intéresse particulièrement à la culture, dimension présente dans notre recherche, mais dont il n'est pas fait mention dans cet article. II nous semblait primordial de faire appel à des experts en didactique de la lecture et de la culture et qui œurrent ailleurs qu'au Québec : leurs connaissances théoriques et pratiques nous ont permis de vérifier l'adéquation du dispositif didactique avec les résultats les plus récents de la recherche théorique.

\section{Collecte des données}

Un questionnaire écrit a été employé pour recueillir les données. Nous avons remis à nos experts le prototype, la maquette de la recherche et le questionnaire. La maquette présente la problématique et le cadre théorique de la recherche. Quant au questionnaire, composé de trois sections, il vise à vérifier la pertinence, la validité, la clarté et la cohérence du prototype. Les deux premières sections contiennent des questions préformées accompagnées d'échelles de Likert, suivies d'espace pour commenter (Lessard-Hébert, Goyette et Boutin, 1996). Composée de six questions, la première section porte sur les assises théoriques du prototype, notamment sur l'exploitation des fondements théoriques sur la lecture littéraire et sa didactique, ainsi que leur adéquation avec le dispositif. La deuxième section comporte 14 questions sur le contenu du dispositif didactique : le réalisme du dispositif didactique en fonction du degré de difficulté des tâches pour les élèves, des notions à l'étude, du découpage dans le temps; le degré de compréhension et de précision des activités, autant pour l'enseignant que pour l'élève; le réalisme en terme d'engagement cognitif de la part des élèves dans les activités proposées; le degré de contrôle par les élèves dans les activités proposées; l'utilité du dispositif didactique pour l'enseignant; son degré d'intérêt pour l'enseignant et pour les élèves. Enfin, la troisième section permet aux experts d'inscrire leurs commentaires généraux. 


\section{Analyse des données}

Les données recueillies ont fait l'objet d'une analyse qualitative de contenu (Bardin, 2007) ; les commentaires, codés sous différentes catégories telles que théorie d'enseignement-apprentissage, culture (qui ne font pas l'objet de cet article), écriture d'invention, questionnement réciproque, écrits de travail, lecture littéraire-compréhension, lecture littéraire-interprétation, dispositif didactique en général, points forts et points à améliorer, ont servi à améliorer le prototype afin d'en arriver à une version « provisoirement » définitive, tant et aussi longtemps que le dispositif didactique n'aura pas été testé en classe, ce qui fera l'objet d'une recherche ultérieure. Afin de limiter les biais possibles, un didacticien du français, également spécialiste de la lecture et chercheur universitaire au Québec, mais non recruté comme expert pour la validation, a également codé les données recueillies et s'est assuré de la prise en compte des commentaires dans la seconde version du prototype.

\section{Résultats et discussion des résultats}

En raison de la nature des données recueillies et de leur portée, qui vise à améliorer le prototype, nous présentons les résultats regroupés, puis nous enchainons directement avec la discussion des résultats en nous concentrant principalement sur les éléments devant faire l'objet d'une amélioration. Certains cours faisant l'objet d'une modification sont indiqués entre parenthèses. Les commentaires recueillis concernent surtout l'interprétation, la pratique de l'écriture d'invention et le dispositif didactique en lui-même.

\section{Le développement de la compréhension et de l'interprétation}

Selon tous les experts, le public cible, c'est-à-dire des élèves qui éprouvent peu de problèmes de compréhension, mais qui ont davantage de mal à interpréter, est bien pris en compte par le dispositif didactique et sera en mesure de réaliser les activités proposées. Selon le deuxième expert, l'enseignant doit contextualiser davantage les apprentissages déjà faits, notamment pour mieux favoriser le développement de la lecture littéraire et ancrer son développement dans les connaissances et les expériences des élèves. Cette contextualisation permettra également de pallier certaines des difficultés rencontrées par les élèves, telles que synthétiser les informations importantes et repérer la structure d'un texte (Van Grunderbeeck et coll., 2004), stratégies de lecture favorisant la compréhension. Dans la version améliorée du dispositif, nous nous sommes assurés que les apprentissages soient davantage mis en contexte et réactualisés, et ce, à intervalles réguliers. Par exemple, lors du premier cours, l'enseignant établit un lien entre les réponses des élèves et leurs connaissances antérieures utiles à la lecture du roman Nikolski (notamment les relais de narration ainsi que les types de narrateurs). II en profite pour rectifier les informations erronées. Puis, à la fin de cette discussion, il demande aux élèves de résumer les informations apprises pendant le cours. Par ailleurs, le deuxième expert, qui « [croit] beaucoup au tutorat réciproque » déplore que cette stratégie ne soit pas employée systé- 
matiquement dans tout le prototype. À cela nous opposons deux arguments. Premièrement, nous ne souhaitons pas accentuer une pesanteur et une lourdeur reconnues au prototype en ajoutant d'autres tâches en équipe qui allongeraient indument la durée de certaines activités, par exemple de rédaction, et en alourdiraient la gestion. Secondement, le but que nous poursuivons avec les activités réalisées en équipe consiste à créer une communauté de lecteurs (Eco, 1985) qui favorisera les échanges des interprétations et la construction de la compréhension (Bucheton et coll., 2004); implanter systématiquement l'apprentissage par les pairs, notamment lors des tâches de rédaction, ne répondrait pas aux deux objectifs didactiques poursuivis par le prototype. II revient évidemment à l'enseignant de s'approprier le dispositif en y apportant les modifications qui lui paraissent souhaitables.

Quant à l'interprétation, les experts soulignent le souci d'acceptation de la complexité de l'interprétation dans l'élaboration du dispositif didactique. Bien que nous relevions des commentaires des experts que le dispositif réponde à l'objectif d'amener les élèves à interpréter un texte littéraire, il ressort des commentaires des deux enseignantes et d'un didacticien une nette insuffisance de la différenciation dans les tâches interprétatives proposées aux élèves, et ce, quelle que soit la pratique didactique employée. En raison de la complexité du roman choisi, Nikolski, et de la grande latitude laissée aux élèves pour explorer différentes interprétations, il convient de différencier davantage : comme le mentionne le troisième expert, «si cette latitude peut s'avérer stimulante pour des élèves plus habiles à interpréter, des élèves faibles ou moins habiles peuvent éprouver des difficultés ». Nous n'avons effectivement pas abordé ce principe d'enseignement dans nos fondements didactiques; nous nous sommes assurés, dans la version améliorée, que certaines tâches conviennent davantage à des élèves plus habiles et d'autres, à des élèves qui éprouvent plus de difficultés interprétatives. Pour les activités liées au questionnement réciproque (cours 2 et cours 5), les élèves pourront choisir des questions qui conviennent à leur niveau d'habileté, ils pourront répondre à une ou plusieurs questions ou encore élaborer leur(s) propre(s) question(s) d’interprétation. Nous proposons également aux élèves plus forts d'aider ceux qui éprouvent davantage de difficultés interprétatives (cours 3). Ici encore, la communauté de lecteurs est mise de l'avant afin d'aider les élèves à interpréter, notamment par la présence de modèles autres que l'enseignant. Tel que le souligne aussi le deuxième expert, « [il] faut prévoir un moyen d'« occuper» les plus rapides (on peut aussi envisager l'entraide entre groupes, pour ceux qui ont mieux compris, ou de confier la tâche d'approbation aux élèves les plus compétents) ». Nous souscrivons à cette remarque. La constitution des équipes est aussi l'occasion de favoriser davantage la différenciation, notamment en constituant des équipes assez homogènes, mais devant accomplir des tâches plus ou moins difficiles selon leur compétence.

Toujours en ce qui concerne l'interprétation, selon quelques-uns des experts, l'accent est surtout mis sur l'interprétation; la compréhension s'avère secondaire dans tout le prototype. Selon les deux enseignantes, il faudrait accroitre les tâches liées à la compréhension. Or, la recension des écrits scientifiques effectuée lors de la définition du problème et l'identification des besoins a permis de constater que de nombreuses pratiques mises en place ne suscitent pas ou alors peu l'activité inter- 
prétative des élèves; voilà pourquoi nous avons mis l'accent, dans le prototype, sur l'interprétation. Rappelons également que tout lecteur doit comprendre le sens du récit pour être en mesure de l'interpréter et de justifier son interprétation (Dufays et coll., 1996). Néanmoins, nous nous sommes assurés, dans la version améliorée du prototype, de mettre en place un peu plus d'activités favorisant la compréhension du roman (cours 1, 2, 5 et 10).

Par ailleurs, que les experts soient enseignants ou didacticiens, ils déplorent que l'enseignant n'agisse que peu souvent comme modèle pour interpréter et qu'il ne donne pas son interprétation du roman. On pourrait alléguer, avec l'expert numéro un, que « la cohérence d'un discours critique explicite de l'enseignant est utile aux élèves pour construire leur propre discours sur l'œuvre»; cependant, toute modélisation a des limites. Au risque de déplaire au premier expert, nous croyons que toute interprétation sur le même roman abordé avec les élèves de la part de l'enseignant doit rester incomplète de peur d'entraver toute liberté interprétative (Falardeau, 2003) de ces derniers. Leur donner voix au chapitre et les rendre habiles à justifier leurs dires constituent des objectifs essentiels. C'est pourquoi nous souhaitons laisser une large place à la liberté interprétative des élèves, tout en les guidant (cours 2). L'enseignant pourrait également donner son interprétation aux élèves et, par le fait même, la modéliser, en s'appuyant sur un autre roman que Nikolski ou alors en ciblant les passages faisant l'objet de son interprétation. Ces commentaires soulèvent, en filigrane, une question. Si l'on met trop l'accent sur l'expérience du lecteur, risque-t-on un certain « dérapage »? Compte tenu des choix didactiques, il se peut que certaines décisions prises désavantagent certains élèves. Selon qu'il choisira des tâches qui privilégient une lecture empathique d'identification ou une lecture distanciée, il faut craindre que le didacticien ou l'enseignant ne puisse répondre aux besoins de tous les élèves. Enfin, il peut s'avérer difficile de « contrôler » les possibles débordements subjectifs des élèves; cela est-il dû au dispositif ou aux limites mêmes de l'interprétation (Eco, 1992)?

\section{L'écriture d'invention, le questionnement réciproque et les écrits de travail}

Quant à la pratique de l'écriture d'invention, les cinq experts s'entendent pour dire qu'elle est bien choisie pour des élèves de la cinquième secondaire. Cependant, l'écriture d'invention devrait davantage être exploitée pour développer la lecture littéraire et la réflexion métacognitive sur celle-ci, notamment en demandant aux élèves de repérer les « effets littéraires » et d'expliquer en quoi ce qu'ont écrit leurs camarades est littéraire, mais aussi en leur demandant d'expliciter en quoi cette pratique permet de mieux s'approprier une démarche de lecture littéraire (deuxième expert). Pour favoriser le développement de la lecture littéraire ainsi qu'une réflexion métacognitive (Le Goff, 2004), nous reprenons, dans la version améliorée du prototype, les pistes de réflexion suggérées par le deuxième expert (cours 13). De plus, trois experts déplorent que la créativité ne fasse pas l'objet d'une évaluation et ceux-ci suggèrent que l'on attribue « une note artistique et une note technique » (deuxième expert). Il est vrai que la qualité d'un texte littéraire tient aussi au fait que ce texte contient des idées développées avec originalité. Dans la grille de correction de l'écriture d'invention, nous ajouterons un critère afin que la créativité soit évaluée, même si cette dernière est fortement limitée 
par les contraintes imposées par cet exercice (cours 10). Par ailleurs, les cinq experts confirment que le questionnement réciproque et les écrits de travail constituent deux pratiques bien choisies pour des élèves de la cinquième secondaire et que, de la façon dont elles sont exploitées, elles permettent de développer la lecture littéraire des élèves.

Ces remarques sur les trois pratiques didactiques choisies amènent à s'interroger sur les pratiques choisies pour développer la lecture littéraire. Certaines pratiques qui auraient pu se révéler plus économiques en terme de temps se sont nécessairement trouvées occultées par nos choix (Van der Maren, 1996). D’ailleurs, deux experts, soit le deuxième et le cinquième, déplorent l'absence de certaines pratiques ou consignes, telles que les cercles de lecture et, pour la pratique de l'écriture d'invention, les consignes de reformulation ou de transposition (par exemple, d'une partie du roman en article de presse). Il est vrai que nous ne les avons pas retenues : certaines d'entre elles sont assez connues et employées, notamment le cercle de lecture qui a fait l'objet de recherches au niveau du primaire et du secondaire (Hébert, 2002; Terwagne, 2003).

\section{Le dispositif didactique, les tâches des élèves et de l'enseignant}

De 11 cours prévus au départ, le dispositif didactique final comporte 13 cours (annexe 1). Les experts soulignent que les élèves sont actifs et engagés cognitivement par le dispositif didactique. De nombreux commentaires provenant tant des enseignants que des didacticiens relèvent que le dispositif didactique, tant dans les activités que dans les évaluations, est particulièrement ambitieux, parfois irréaliste : les élèves « peuvent trouver lassant et répétitif de travailler aussi longtemps un roman » (quatrième expert). Le prototype élaboré dans sa première version est effectivement trop long. Les activités incontournables au développement de la lecture littéraire ont donc été séparées des activités qui semblent facultatives. En outre, il se dégageait des remarques des enseignantes que les évaluations sont trop nombreuses et alourdissent la tâche de l'enseignant qui souhaiterait mettre en place ce dispositif didactique. Nous avons donc réduit les évaluations, notamment en omettant une des deux évaluations sommatives sur l'écriture d'invention.

Enfin, il ressort de l'analyse des commentaires qu'il faut préciser davantage les tâches, les priorités et la manière dont l'enseignant anime les activités : « définir les tâches à faire par les élèves est important, mais prévoir aussi celles de [l'enseignant] et les contenus d'intervention l'est aussi » (premier expert). Dans la version améliorée, nous avons spécifié davantage les tâches de l'enseignant et la manière de les accomplir. Par exemple, lors du cours sur la littérarité (cours 6), nous avons fourni des questions que peut poser l'enseignant à ses élèves. Afin de le guider dans ses réflexions et ses interventions, nous lui proposons aussi quelques informations sur les notions qu'il aurait à aborder. Cependant, tout dispositif didactique, si complet soit-il, ne saurait répondre de façon exhaustive aux divers aspects de l'hétérogénéité du milieu (économique, culturel, ethnique, linguistique...), des élèves et de l'enseignant. Toute didactique est « virtuelle » : elle est pensée en fonction d'élèves précis qui ont sensiblement tous le même niveau de connaissances, voire même en fonction d'une 
représentation des élèves qui peut parfois être irréelle (par exemple, méconnaissance de leurs préoccupations ou de leur motivation), d'un espace-temps parfait dans lequel il y a peu d'imprévus. Enfin, les interactions entre les élèves et avec l'enseignant jouent un rôle déterminant que la didactique ne pourrait entièrement prévoir (Halté, 1992).

De l'analyse des données se dégagent deux constatations majeures qui dépassent largement le cadre du dispositif didactique élaboré. D'une part, le choix des œuvres avec lesquelles développer la lecture littéraire pose encore problème, tant pour les didacticiens que pour les enseignants. Quelles œuvres proposer? Bien que la littérature puisse « nous faire mieux comprendre le monde » et « nous aider à vivre » (Todorov, 2007, p. 72), certains élèves peuvent ne pas être touchés ou interpelés par les textes donnés à lire en raison, par exemple, d'intérêts différents, voire divergents de la majorité de la classe, d'une absence de motivation, de difficultés de compréhension qui freinent l'identification au récit... Si l'enseignant part des intérêts de ses élèves pour sélectionner des œuvres (Zakhartchouk, 1999), doit-on craindre d'oublier certains textes qui piqueraient quand même leur curiosité? Si l'enseignant contribue à transmettre une culture, quel équilibre doit-on garder entre le familier et la nouveauté (Falardeau et Simard, 2007)? De surcroit, la didactique de la lecture littéraire pose problème, comme nous l'avons souligné dans la problématique : dans le contexte scolaire, avec quelles expériences, représentations et connaissances l'élève aborde-t-il sa lecture littéraire d'un texte? Comment en tenir compte concrètement? Comment ce bagage bonifie-t-il, transforme-t-il sa lecture littéraire? Entre la nécessité de développer la lecture littéraire et d'évaluer, est-ce possible d'exploiter cet « achèvement » par le lecteur, d'exploiter le texte du lecteur (Trouvé, 2004)?

\section{Conclusion}

Par cette recherche-développement, nous souhaitions élaborer un dispositif didactique susceptible de développer la compréhension et l'interprétation des élèves du secondaire. Par l'utilisation de ce dispositif didactique amélioré à la suite des commentaires des experts, il reste à souhaiter que des retombées puissent être observées chez les élèves, quel que soit leur milieu, sous l'aspect d'une compétence plus grande en lecture littéraire : facilité accrue à lire un texte littéraire, facilité à expliquer un texte littéraire. Bien que, dans l'immédiat, le dispositif ne fasse pas l'objet d'une expérimentation, nous croyons qu'ainsi modifié, il répond aux besoins des enseignants et des élèves dans le développement de la lecture littéraire. Néanmoins, ce dispositif devra faire l'objet d'une mise en pratique formelle dans une recherche ultérieure : il existe un écart difficilement contrôlable entre ce qu'un enseignant planifie réaliser avec ses élèves et ce qu'il réalise concrètement en classe. En outre, plusieurs paramètres ont influencé la création de ce dispositif et devraient être pris en compte lors de sa mise à l'essai : les aptitudes moyennes des élèves ciblés, leur familiarité avec ce genre d'activités, l'utilisation qui en est faite (à des fins d'évaluation sommative ou encore formative) ainsi que le moment de l'année où l'enseignant met en place le dispositif. C'est en alliant recherches théoriques, recherches-développements et recherches empiriques que des pratiques et outils efficaces seront proposés pour développer la lecture littéraire. 


\section{Références}

Bardin, L. (2007). L'analyse de contenu. Paris : Presses universitaires de France.

Beaudry, M.-C. (2009). Enseigner les stratégies de lecture littéraire au secondaire: une recherche-développement autour du roman «Nikolski » de Nicolas Dickner (Thèse de doctorat inédite). Université de Montréal.

Beaudry, M.-C. et Huneault, M. (2011). L'écriture d'invention pour développer le sujet lecteur de texte littéraire chez des élèves de la cinquième secondaire. Dans M.-C. Beaudry, I. Carignan et N. Lacelle (dir.), Revue pour la recherche en éducation (p. 84-97). Actes de colloque de l'ACFAS. Repéré à http://revue-recherche-education.com/Acfas_2010.php

Bourdieu, P. et Passeron, J.-C. (1985). Les héritiers : les étudiants et la culture. Paris : De Minuit.

Bucheton, D., Brénas, Y., Chabanne, J.-C. et Dupuy, C. (2004). Parier sur la créativité. Cahiers pédagogiques, 420, 11-13.

Chabanne, J.-C. et Bucheton, D. (2002). L'activité réflexive dans les écrits intermédiaires : quels indicateurs? Dans J.-C. Chabanne et D. Bucheton (dir.), Parler et écrire pour penser, apprendre et se construire: l'écrit et l'oral réflexifs (p. 25-51). Paris : Presses universitaires de France.

Daunay, B. et Denizot, N. (2003). Construction d'un « objet didactique » : l'écriture d'invention au collège et au lycée. Compte-rendu d'une recherche en cours. Les cahiers Théodile, 4, 81-101.

De Beaudrap, A.-R. et Benoit, J.-P. (1999). Quand des lycéens commentent un texte : bilan des nouvelles épreuves du commentaire littéraire au baccalauréat. Enjeux, 46, 116-128.

Dezutter, O., Larivière, I., Bergeron, M.-D. et Morissette, C. (2007). Les pratiques déclarées des enseignants québécois dans la sélection et l'exploitation des œuvres complètes inscrites au programme de lecture des élèves. Dans É. Falardeau, C. Fisher, C. Simard et N. Sorin (dir.), La didactique du français: les voies actuelles de la recherche (p. 83-100). Québec: Les Presses de l'Université Laval.

Dickner, N. (2007). Nikolski. Québec: Alto.

Dufays, J.-L. (2006). La lecture littéraire, des « pratiques du terrain » aux modèles théoriques. Lidil, 33, 79-101.

Dufays, J.-L. (1997). Lire au pluriel. Pour une didactique de la diversité des lectures à l'usage des 14-15 ans. Pratiques, 95, 31-52.

Dufays, J.-L. (1996). Culture/compétence/plaisir : la nécessaire alchimie de la lecture littéraire. Dans J.-L. Dufays, L. Gemenne et D. Ledur (dir.), Pour une lecture littéraire 2, Bilan et confrontations (p. 167-175). Actes du colloque de Louvain-la-Neuve, Bruxelles: De Boeck et Duculot.

Dufays, J.-L., Gemenne, L. et Ledur, D. (1996). Pour une lecture littéraire 2, Bilan et confrontations. Actes du colloque de Louvain-la-Neuve, Bruxelles : De Boeck et Duculot.

Dumortier, J.-L. (1991). Questionnaires en question. Enjeux, 23, 71-87. 
Eco, U. (1992). Les limites de l'interprétation. Paris : Grasset.

Eco, U. (1985). Lector in fabula : le rôle du lecteur. Paris : Grasset.

Falardeau, É. (2003). Compréhension et interprétation : deux composantes complémentaires de la lecture littéraire. Revue des sciences de l'éducation, 29(3), 673-694.

Falardeau, É. et Simard, D. (2007). Le rapport à la culture des enseignants de français et son rôle dans l'articulation de la culture avec les contenus disciplinaires. Dans É. Falardeau, C. Fisher, C. Simard et N. Sorin. (dir.), La didactique du français. Les voies actuelles de la recherche (p. 147-164). Québec: Presses de l'Université Laval.

Fayol, M. (1992). Comprendre ce qu'on lit: de l'automatisme au contrôle. Dans M. Fayol, J-E. Gombert, P. Lecocq et L. Sprenger-Charolles (dir.), Psychologie cognitive de la lecture (p. 73-105). Paris: Presses universitaires de France.

Gervais, B. (2006). À l'écoute de la lecture. Québec: Nota Bene.

Gervais, B. et Bouvet, R. (2007). Introduction. Dans B. Gervais et R. Bouvet (dir.), Théories et pratiques de la lecture littéraire (p. 1-7). Québec: Presses de l'Université du Québec.

Guichon, N. (2007). Recherche-développement et didactique des langues. Les Cahiers de l'Acedle, Journées $\mathrm{NeQ}$, Méthodologie de recherche en didactique des langues, 4. Repéré à http://hal.archives-ouvertes.fr/docs/00/35/58/13/PDF/Guichon_r_d2007.pdf

Halté, J.-F. (1992). La didactique du français. Paris : Presses universitaires de France.

Hébert, M. (2002). Co-élaboration du sens dans les cercles littéraires entre pairs en première secondaire : étude des relations entre les modalités de lecture et de collaboration (Thèse de doctorat inédite). Université de Montréal.

Houdart-Merot, V. (2004). Les enjeux de l'écriture d'invention. Cahiers pédagogiques, 420, $37-38$.

Huyhn, J.-A. (2004). Écriture d'invention et « identité » du sujet lecteur. Dans A. Rouxel et G. Langlade (dir.), Le sujet lecteur: lecture subjective et enseignement de la littérature (p. 305-316). Rennes: Presses universitaires de Rennes.

Iser, W. (1985). L'acte de lecture : théorie de l'effet esthétique. Bruxelles: Pierre Mardaga.

Jauss, H. R. (1978). Pour une esthétique de la réception. Paris : Gallimard.

Jouve, V. (1993). La lecture. Paris : Hachette.

Langlade, G. (2007a). L'activité fictionnalisante du lecteur. Modernités, 23. Repéré à http://www.uhb.fr/labos/celam/didactique/langlade.pdf

Langlade, G. (2007b). La lecture subjective. Québec français, 145, 71-73.

Langlade, G. (2002). Lire des œuvres intégrales au collège et au lycée. Toulouse : CRDP Midi-Pyrénées.

Langlade, G. (2001). Et le sujet lecteur dans tout ça? Enjeux, 51-52, 53-62. 
Lebrun, M. (1996). Littérature de jeunesse et journal dialogué : les deux font la paire. Dans P.-M. Beaude, A. Petitjean et J.-M. Privat (dir.), La scolarisation de la littérature de jeunesse (p. 347-358). Actes du colloque international «La scolarisation de la littérature de jeunesse : enjeux et effets », Metz : Université de Metz.

Lebrun, N. et Berthelot, S. (1994). Plan pédagogique : une démarche systématique de planification de l'enseignement. Ottawa : Nouvelles AMS.

Lebrun, M. et Le Pailleur, M. (1992). De la lecture efférente à la lecture esthétique des récits. Dans C. Préfontaine et M. Lebrun (dir.), La lecture et l'écriture, enseignement et apprentissage (p. 183200). Montréal : Logiques.

Le Goff, F. (2004). Métadiscours en écriture d'invention et modes d'investissement du sujet lecteur. Dans A. Rouxel et G. Langlade (dir.), Le sujet lecteur : lecture subjective et enseignement de la littérature (p. 293-304). Rennes : Presses universitaires de Rennes.

Lessard-Hébert, M., Goyette, G. et Boutin, G. (1996). La recherche qualitative: fondements et pratiques. Montréal : Éditions Nouvelles.

Loiselle, J. (2001). La recherche-développement en éducation : sa nature et ses caractéristiques. Dans M. Anadón (dir.), Nouvelles dynamiques de recherche en éducation (p. 77-97). Sainte-Foy: Presses de l'Université Laval.

Loiselle, J. et Harvey, S. (2007). La recherche-développement en éducation : fondements, apports et limites. Recherches qualitatives, 27(1), 40-59.

Manzo, A. V. (1969). The ReQuest procedure. Journal of Reading, 13(2), 123-126.

Ministère de l'Éducation, du Loisir et du Sport, MÉLS (2005). Les profils de lecteurs chez les jeunes du secondaire. Rapport d'action concertée pour le soutien à la recherche en lecture.

Montballin, M. (1999). La compréhension des textes littéraires : attitudes «à risque » et procédures régulatrices. Enjeux, 46, 57-73.

Pamfil, A. (2007). Une mise en forme de la compétence littéraire. Le français aujourd'hui, 157, 101-109.

Petitjean, A. (2002). Écriture d'invention et nouveaux programmes des lycées. Dans J.-F. Inisan et É. Nonnon (dir.), Écriture et invention (p. 19-31). IUFM Nord - Pas de Calais - Centre d'Arras. Repéré à http://www.lille.iufm.fr/IMG/pdf/1_-_Mise_en_perspective.pdf

Picard, M. (1986). La lecture comme jeu. Paris : Minuit.

Poslaniec, C. (2002). Vous avez dit « littérature »? Paris : Hachette.

Privat, J.-M. (2005). Socio-logiques des didactiques de la lecture. Dans J.-L. Chiss, J. David et Y. Reuter (dir.), Didactique du français : fondements d'une discipline (p. 119-134). Bruxelles : De Boeck.

Rosenblatt, L. M. (1995). Literature as exploration. New York: Modern Language Association of America. 
Rouxel, A. (2007). Pratiques de lecture : quelles voies pour favoriser l'expression du sujet lecteur? Le français d'aujourd'hui, 157, 65-73.

Rouxel, A. (2002). Qu'entend-on par lecture littéraire? Dans C. Tauveron (dir.), La lecture et la culture littéraires au cycle des approfondissements (p. 12-22). Paris: Actes de l'université d'automne. Repéré à http://www.eduscol.education.fr/D0126/lecture_culture_litteraires.pdf

Rouxel, A. (1996). Enseigner la lecture littéraire. Rennes: Presses universitaires de Rennes.

Stolovitch, H. D. et LaRocque, G. (1983). Introduction à la technologie de l'instruction. Saint-Jean-surRichelieu : Éditions Préfontaine.

Tauveron, C. (2002). Lire la littérature à l'école : pourquoi et comment conduire cet apprentissage spécifique? Paris : Hatier.

Tauveron, C. (2001). Relations conjugales dans le couple infernal compréhension/interprétation: " un autre drame très parisien ». Dans C. Tauveron (dir.), Comprendre et interpréter le littéraire à l'école et au-delà (p. 5-24). Paris: INRP.

Terwagne, S. (2003). Les cercles de lecture : interagir pour développer ensemble des compétences de lecteurs. Bruxelles: De Boeck.

Thérien, M. (1997). De la définition du littéraire et des œuvres à proposer aux jeunes. Dans M. NoëlGaudreault (dir.), Didactique de la littérature : bilans et perspectives (p. 19-32). Montréal : Nuit blanche.

Todorov, T. (2007). La littérature en péril. Paris : Flammarion.

Trouvé, A. (2004). Le roman de la lecture. Critique de la raison littéraire. Wavre : Pierre Mardaga.

Vandendorpe, C. (1992). Comprendre et interpréter. Dans C. Préfontaine et M. Lebrun (dir.), La lecture et l'écriture, enseignement et apprentissage (p. 159-181). Montréal : Logiques.

Van Grunderbeeck, N., Théorêt, M., Cartier, S., Chouinard, R. et Garon, R. (2004). Étude longitudinale et transversale des conditions scolaires favorables au développement des habitudes et des compétences en lecture chez des élèves du secondaire, rapport final, Action concertée du Fonds FCAR-CQRS-MCC-MEQ-MFE, Programme pour le soutien de la recherche en lecture, Montréal, Université de Montréal.

Van der Maren, J.-M. (1996). Méthodes de recherche pour l'éducation. Montréal : Les Presses de l'Université de Montréal.

Zakhartchouk, J.-M. (1999). L'enseignant, un passeur culturel. Paris : E.S.F. 


\section{Annexe}

\section{Annexe 1 - Version améliorée du dispositif didactique}

Voici le contenu exhaustif des 13 cours élaborés à la suite des commentaires des experts. Le matériel fourni est ici absent. Les cours qui peuvent être facultatifs sont indiqués; il revient à l'enseignant de faire le dispositif didactique au complet (13 cours) ou de ne réaliser que les tâches liées aux écrits de travail (cours 6 à 9) ou celles de l'écriture d'invention (cours 10 à 13).

\section{Cours 1 : Entrée dans la lecture littéraire de Nikolski}

\begin{tabular}{|c|c|}
\hline Pratique & Questionnement réciproque \\
\hline Composante de la lecture littéraire sollicitée & Compréhension, interprétation \\
\hline TÂCHES DE L'ENSEIGNANT & TÂCHES DES ÉLÈVES \\
\hline $\begin{array}{l}\text { 1. Écrire au tableau les titres de films et de romans suivants. Ques- } \\
\text { tionner les élèves sur le potentiel significatif de ces titres populaires } \\
\text { et moins populaires. Titres: Titanic, Harry Potter et la chambre des } \\
\text { sorciers, Le Diner de cons, Star Wars, Bon cop bad cop, The Godfather, } \\
\text { C.R.A.Z.Y, Quatre filles et un jeans,... Questions : D’après vous, quel est le } \\
\text { récit pour chacun de ces titres? Ces derniers sont-ils représentatifs du } \\
\text { contenu du film ou du roman? Nous aident-ils à comprendre l'histoire } \\
\text { racontée? }\end{array}$ & $\begin{array}{l}\text { 1. Puiser dans leurs connaissances antérieures, dans leur culture, afin } \\
\text { de répondre aux questions posées par l'enseignant. }\end{array}$ \\
\hline $\begin{array}{l}\text { 2. À partir du titre du roman Nikolski, poser la question suivante aux } \\
\text { élèves: Que vous suggère le titre du roman que nous allons lire, } \\
\text { Nikolski? Demander aux élèves de justifier leurs réponses. }\end{array}$ & $\begin{array}{l}\text { 2. Anticiper le récit à partir du titre du roman, partager et justifier leurs } \\
\text { opinions. }\end{array}$ \\
\hline $\begin{array}{l}\text { 3. Distribuer le document intitulé « } 1.1 \text { Avant de lire Nikolski » et deman- } \\
\text { der de répondre individuellement aux cinq questions. Il est également } \\
\text { possible de demander aux élèves de lire à haute voix les incipits des } \\
\text { différentes parties du roman, puis de répondre collectivement ou indivi- } \\
\text { duellement aux questions. }\end{array}$ & $\begin{array}{l}\text { 3. À l'aide du roman et du document «1.1 Avant de lire Nikolski », } \\
\text { prendre connaissance de la table des matières et des incipits des } \\
\text { chapitres sélectionnés afin de mieux anticiper le contenu du roman, } \\
\text { puis répondre par écrit, individuellement, aux cinq questions (sur le } \\
\text { document } 1.1 \text { ou sur des feuilles). }\end{array}$ \\
\hline $\begin{array}{l}\text { 4. Collectivement, demander aux élèves de partager leurs réponses. } \\
\text { Au fur et à mesure que les élèves répondent, établir un lien avec les } \\
\text { connaissances déjà apprises et qui sont utiles à la lecture de Nikolski : } \\
\text { les relais de narration (ce qui explique la présence de trois narrateurs } \\
\text { différents), les types de narrateurs (absent, présent), les principaux lieux } \\
\text { où se déroule le récit. Rectifier également les informations erronées. Il } \\
\text { importe de demander aux élèves de noter ces informations pertinentes } \\
\text { pour la compréhension du récit. À la fin de cette discussion, résumer } \\
\text { les informations ou demander à un élève de le faire : « Nous lirons un } \\
\text { roman qui se déroule dans plusieurs lieux en Amérique et qui met en } \\
\text { scène trois personnages principaux, soit « Je », Joice et Noah... ». }\end{array}$ & $\begin{array}{l}\text { 4. Partager leurs réponses et noter les informations supplémentaires } \\
\text { fournies par l'enseignant (sur le document } 1.1 \text { ou sur des feuilles). }\end{array}$ \\
\hline $\begin{array}{l}\text { 5. Distribuer le document «1.2 Pour bien comprendre Nikolski». Pré- } \\
\text { senter les cartes géographiques et situer Nikolski, Tête-à-la-baleine et } \\
\text { le marché Jean-Talon. Puis, présenter la biographie de Nicolas Dickner. } \\
\text { Ensuite, expliciter les stratégies de compréhension et d'interprétation } \\
\text { qui peuvent aider lors de la lecture. Allouer deux semaines pour la } \\
\text { lecture du roman. }\end{array}$ & $\begin{array}{l}\text { 5. Prendre connaissance, en classe et à la maison, des cartes géogra- } \\
\text { phiques. Identifier les principaux lieux du roman sur les cartes. Utiliser } \\
\text { les stratégies de compréhension et d'interprétation s'ils éprouvent des } \\
\text { difficultés dans leur lecture (tout ceci se trouve dans le document 1.2). } \\
\text { Les élèves disposent de deux semaines pour lire le roman. }\end{array}$ \\
\hline
\end{tabular}




\section{Cours 2 : Étude de Nikolski}

\begin{tabular}{|c|c|}
\hline Pratique & Questionnement réciproque \\
\hline Composante de la lecture littéraire sollicitée & Compréhension, interprétation \\
\hline TÂCHES DE L'ENSEIGNANT & TÂCHES DES ÉLÈVES \\
\hline $\begin{array}{l}\text { 1. La première activité vise à montrer aux élèves un modèle du travail } \\
\text { à effectuer. Il est important de le mentionner. - Lire, avec les élèves, le } \\
\text { passage de la page } 17 \text { dans lequel il est question de Richard Brautigan. - } \\
\text { Demander s'ils connaissent Richard Brautigan, si des éléments du texte } \\
\text { permettent de deviner qui est cet homme (on devine qu'il est poète : " } \\
\text { [...] elle offrait aux passants des poèmes de Richard Brautigan [...]»). - } \\
\text { Demander quelle stratégie de compréhension les a aidés à trouver cette } \\
\text { réponse (à l'aide du document «1.2 Pour bien comprendre Nikolski », } \\
\text { remis au cours 1). }\end{array}$ & $\begin{array}{l}\text { 1. Approfondir l'allusion à Richard Brautigan : lire un passage du } \\
\text { roman et repérer les éléments qui permettent de dire qui est Brau- } \\
\text { tigan (en consultant le document } 1.2 \text { remis au cours } 1 \text { ). Puis, lire les } \\
\text { diverses informations sur cet auteur et la beat generation et relever les } \\
\text { informations pertinentes pour expliquer cette allusion (document 2.1). } \\
\text { Finalement, interroger sa signification et sa portée. }\end{array}$ \\
\hline $\begin{array}{l}\text { - Distribuer le document « } 2.1 \text { Informations sur l'allusion à Richard } \\
\text { Brautigan » et préciser qu'il contient le fruit de recherches. Laisser le } \\
\text { temps de lire (individuellement ou collectivement). }\end{array}$ & \\
\hline $\begin{array}{l}\text { - Demander aux élèves de résumer oralement les informations retenues } \\
\text { sur Richard Brautigan (" étranges recueils de poèmes », années cin- } \\
\text { quante et soixante, San Francisco, écriture originale...) et la beat gene- } \\
\text { ration (côte ouest américaine, mouvement poétique, révolte, hippies...) } \\
\text { et les noter au tableau. }\end{array}$ & \\
\hline $\begin{array}{l}\text { - Questionner les élèves : L'effet aurait-il été le même si Dickner avait } \\
\text { écrit que la mère de " Je » vendait des poèmes de Victor Hugo? Pourquoi? } \\
\text { Quel est le parallèle qui existe entre la mère de « Je » et Richard Brautigan } \\
\text { (contemporains, l'un rédige de la poésie, l'autre la vend, tous les deux } \\
\text { font partie du même mouvement...)? }\end{array}$ & \\
\hline $\begin{array}{l}\text { - Faire remarquer la présence des références, des textes sources et la } \\
\text { documentation variée. }\end{array}$ & \\
\hline $\begin{array}{l}\text { 2. Distribuer le document « } 2.2 \text { Questionnaire sur Nikolski » et présenter } \\
\text { le travail à réaliser lors du prochain cours : - Sélectionner une question; } \\
\text { - Effectuer une recherche afin d’y répondre; } \\
\text { - Préparer une mini-leçon en exploitant les documents sources de la } \\
\text { même manière que l'a fait l'enseignant. }\end{array}$ & $\begin{array}{l}\text { 2. Prendre connaissance du document « } 2.2 \text { Questionnaire sur Nikolski» } \\
\text { et compléter les consignes si les élèves le jugent nécessaire. }\end{array}$ \\
\hline $\begin{array}{l}\text { 3. Demander aux élèves de se regrouper en équipe de deux (l'ensei- } \\
\text { gnant peut laisser les élèves se placer avec qui ils veulent ou alors } \\
\text { imposer des coéquipiers), puis de choisir une question en fonction de } \\
\text { leurs intérêts et habiletés. Les élèves qui le désirent peuvent choisir } \\
\text { deux questions. Celle-ci doit être donnée à l'enseignant (une équipe par } \\
\text { question). Préciser que le prochain cours, qui aura lieu à la biblio- } \\
\text { thèque, sera consacrè à la recherche et à l'élaboration de la réponse. }\end{array}$ & $\begin{array}{l}\text { 3. Se regrouper en équipe de deux, puis choisir une question en fonc- } \\
\text { tion de leurs intérêts et de leurs habiletés (à l'aide du document 2.2). }\end{array}$ \\
\hline
\end{tabular}




\section{Cours 3 : Mise en commun du travail d'étude de Nikolski}

\begin{tabular}{|c|c|}
\hline Pratique & Questionnement réciproque \\
\hline Composante de la lecture littéraire sollicitée & Compréhension, interprétation \\
\hline TÂCHES DE L'ENSEIGNANT & TÂCHES DES ÉLÈVES \\
\hline $\begin{array}{l}\text { 1. Rappeler les tâches à réaliser pendant le cours, qui se déroule, idéale- } \\
\text { ment, à la bibliothèque: } \\
\text { - Trouver les textes sources évoqués par Dickner et prendre en note les } \\
\text { références bibliographiques; } \\
\text { - Élaborer sa réponse en expliquant, en établissant des liens avec le } \\
\text { roman et les textes sources (selon le modèle présenté en classe lors du } \\
\text { cours 2); } \\
\text { - S’il reste du temps, sinon ce sera à réaliser à la maison, élaborer la } \\
\text { mini-leçon: qui dira quoi, comment... }\end{array}$ & $\begin{array}{l}\text { 1. Trouver des documents qui permettent de répondre à la question (ou } \\
\text { les questions, le cas échéant) dont les élèves sont responsables, puis } \\
\text { élaborer la réponse. Si le temps le permet, créer la mini-leçon, sinon, } \\
\text { terminer le tout à la maison (à l'aide du document « } 2.2 \text { Questionnaire } \\
\text { sur Nikolski »). }\end{array}$ \\
\hline $\begin{array}{l}\text { 2. Circuler et aider les élèves à parfaire leur compréhension et interpré- } \\
\text { tation. }\end{array}$ & $\begin{array}{l}\text { 2. Les élèves qui auraient terminé le travail avant la fin de la période } \\
\text { montrent leur résultat à l'enseignant et aident les autres dans leurs } \\
\text { recherches. }\end{array}$ \\
\hline
\end{tabular}

\section{Cours 4 : Mise en commun du travail d'étude de Nikolski}

\begin{tabular}{|l|l|}
\hline Pratique & Questionnement réciproque \\
\hline Composante de la lecture littéraire sollicitée & Compréhension, interprétation \\
\hline \multicolumn{1}{|c|}{ TÂCHES DE L'ENSEIGNANT } & TÂCHES DES ÉLÈVES \\
\hline $\begin{array}{l}\text { 1. Rappeler la tâche à réaliser pendant le cours : présenter à tour de } \\
\text { rôle la mini-leçon préparée par l'équipe en suivant l'ordre des questions } \\
\text { (document 2.2). Rectifier, soulever d'autres questions... }\end{array}$ & $\begin{array}{l}\text { 1. Présenter devant la classe la mini-leçon. Pendant ce temps, les autres } \\
\text { leurs camarades et l'enseignant). }\end{array}$ \\
\hline
\end{tabular}

\section{Cours 5 : Questionnement}

\begin{tabular}{|c|c|}
\hline Pratique & Questionnement réciproque \\
\hline Composante de la lecture littéraire sollicitée & Compréhension, interprétation \\
\hline TÂCHES DE L'ENSEIGNANT & TÂCHES DES ÉLÈVES \\
\hline $\begin{array}{l}\text { 1. Présenter les consignes: les élèves disposent d'une dizaine de } \\
\text { minutes pour rédiger, en équipe de deux élèves, une ou deux questions } \\
\text { qui permettent de comprendre et d'interpréter le roman. Ces questions } \\
\text { seront soumises aux élèves de la classe pendant la période. }\end{array}$ & $\begin{array}{l}\text { 1. Prendre connaissance de la tâche à réaliser. Ensuite, lire les docu- } \\
\text { ments } 1.2 \text { (remis au cours 1) et } 5.1 \text { et formuler, collectivement, une } \\
\text { question qui sert de modèle. }\end{array}$ \\
\hline $\begin{array}{l}\text { - Tout d'abord, demander de prendre le document « } 1.2 \text { Pour bien } \\
\text { comprendre Nikolski» remis au cours } 1 \text { et d'aller à la section sur les } \\
\text { stratégies de compréhension et d'interprétation. Puis, distribuer « } 5.1 \\
\text { Balises pour formuler des questions sur Nikolski». Il est important de } \\
\text { mentionner que ces deux documents présentent des pistes pour formu- } \\
\text { ler des questions. }\end{array}$ & \\
\hline $\begin{array}{l}\text { - À l'aide de ces documents, demander de formuler, collectivement, } \\
\text { un exemple de questions. Noter cette question au tableau (la question } \\
\text { formulée sert de modèle : elle ne doit pas demander aux élèves de } \\
\text { repérer). }\end{array}$ & \\
\hline $\begin{array}{l}\text { 2. Laisser une dizaine de minutes pour que les élèves rédigent une ou } \\
\text { deux questions. Approuver les questions et demander aux élèves plus } \\
\text { habiles d'aider les autres. }\end{array}$ & $\begin{array}{l}\text { 2. À l'aide des deux documents et du roman, composer une ou deux } \\
\text { questions de compréhension ou d'interprétation. La soumettre ensuite } \\
\text { à l'enseignant. }\end{array}$ \\
\hline $\begin{array}{l}\text { 3. Inviter à tour de rôle les équipes à présenter leur question, qui peut } \\
\text { être notée au tableau. En profiter pour rectifier, soulever d'autres } \\
\text { questions... }\end{array}$ & $\begin{array}{l}\text { 3. Collectivement, répondre aux questions formulées. Questionner de } \\
\text { nouveau, apporter des précisions... }\end{array}$ \\
\hline
\end{tabular}




\section{Cours 6 : Réflexion sur le processus de littérarisation (cours facultatif)}

\begin{tabular}{|c|c|}
\hline Pratique & Questionnement, écrits de travail \\
\hline Composante de la lecture littéraire sollicitée & Compréhension, interprétation \\
\hline TÂCHES DE L'ENSEIGNANT & TÂCHES DES ÉLÈVES \\
\hline \multicolumn{2}{|l|}{$\begin{array}{l}\text { Auparavant, consulter le document « } 6.1 \text { Notes sur le texte littéraire, la } \\
\text { lecture littéraire et les prix reçus par le roman Nikolski » pour obtenir } \\
\text { quelques informations sur ces notions et alimenter sa propre réflexion. }\end{array}$} \\
\hline $\begin{array}{l}\text { 1. Annoncer la tâche : discussion sur ce qu'est un texte littéraire, une } \\
\text { lecture littéraire et un prix. Cette discussion permettra de rédiger un } \\
\text { commentaire critique. } \\
\text { - Demander aux élèves de se regrouper en équipe de quatre et de } \\
\text { choisir 1) qui rapportera leurs propos, 2) qui gardera le temps et 3) qui } \\
\text { posera les questions à l'enseignant, ceci afin de mener une discussion } \\
\text { efficace. } \\
\text { - Laisser une vingtaine de minutes pour répondre aux deux questions } \\
\text { qui suivent : Selon vous, qu'est-ce qu'un texte littéraire? Selon vous, } \\
\text { qu'est-ce qu'une lecture littéraire? Donnez des exemples, détaillez. } \\
\text { - Proposer les pistes suivantes pour alimenter la réflexion : Quel est le } \\
\text { rapport qu'entretient un texte littéraire avec le genre littéraire dans lequel } \\
\text { il s'inscrit (innovation, tradition, présences ou non de formes types...)? } \\
\text { Reconnait-on la présence ou non, à un certain degré, de l'intertextualité } \\
\text { sous forme, par exemple, d'allusions, de commentaires, de parodies? } \\
\text { Quel est l'accueil réservé au texte littéraire par la critique, la communauté } \\
\text { de lecteurs, les enseignants... et la valeur qui lui est accordée, l'impact du } \\
\text { texte littéraire sur l'imaginaire du lecteur, d'une communauté de lecteurs? }\end{array}$ & $\begin{array}{l}\text { 1. En équipe de quatre, choisir celui qui rapportera les propos lors de } \\
\text { la plénière, celui qui s'assurera que le temps est bien exploité et celui } \\
\text { qui posera des questions à l'enseignant si nécessaire. Puis, réfléchir à } \\
\text { ce qu'est un texte littéraire et une lecture littéraire. Noter les éléments } \\
\text { trouvés. }\end{array}$ \\
\hline $\begin{array}{l}\text { 2. En grand groupe, animer une plénière : les élèves-rapporteurs } \\
\text { mettent en commun les éléments trouvés. Il est important de leur faire } \\
\text { découvrir qu'il existe un flou, une absence de critères précis et stables, } \\
\text { et ce, tant pour le texte littéraire que pour la lecture littéraire. }\end{array}$ & $\begin{array}{l}\text { 2. En grand groupe, partager les informations trouvées. Compléter les } \\
\text { notes, questionner... }\end{array}$ \\
\hline $\begin{array}{l}\text { 3. Poursuivre la discussion en interrogeant les élèves sur les éléments } \\
\text { suivants: Selon vous, quels sont les critères pour attribuer un prix à un } \\
\text { livre? Est-ce qu'attribuer un prix à un texte signifie que ce texte est litté- } \\
\text { raire? Quelles sont les conséquences? } \\
\text { Il est important de faire comprendre aux élèves que prix ne signifie pas } \\
\text { nécessairement littérarité et qu'il n'existe pas de critères définitifs pour } \\
\text { définir ce qui est littéraire et ce qui ne l'est pas. De plus, il faut leur faire } \\
\text { comprendre que les critères pour attribuer des prix ne sont jamais très } \\
\text { précis. }\end{array}$ & 3. Répondre aux questions de l'enseignant et compléter les notes. \\
\hline $\begin{array}{l}\text { 4. Demander aux élèves de répondre individuellement à la question } \\
\text { suivante, sur laquelle ils travailleront lors du cours } 7 \text {. Préciser que ceci } \\
\text { est l'étape du remue-méninge. Selon vous, Nikolski mérite-t-il un ou des } \\
\text { prix littéraires? Pourquoi en mérite-t-il? Pourquoi n'en mérite-t-il pas? } \\
\text { Donnez des éléments précis du roman. Vous pouvez le comparer avec } \\
\text { d'autres romans. }\end{array}$ & $\begin{array}{l}\text { 4. Noter la question donnée et commencer la réflexion sous forme de } \\
\text { remue-méninges. Poursuivre cette étape à la maison. }\end{array}$ \\
\hline
\end{tabular}




\section{Cours 7 et 8 : Rédaction d'un commentaire critique sur Nikolski (cours facultatifs)}

\begin{tabular}{|c|c|}
\hline \multicolumn{1}{|c|}{ Pratique } & \multicolumn{1}{|c|}{ Écrits de travail } \\
\hline \multicolumn{1}{|c|}{ Composante de la lecture littéraire sollicitée } & \multicolumn{1}{c|}{ Compréhension, interprétation } \\
\hline \multicolumn{1}{|c|}{ TÂCHES DE L'ENSEIGNANT } & \multicolumn{1}{c|}{ TÂCH DES ÉLÈVES } \\
\hline $\begin{array}{l}\text { 1. Présenter le travail à réaliser : poursuivre la réflexion sur Nikolski à } \\
\text { partir du document «7.1 Nikolski mérite-t-il des prix? ». Remettre ce } \\
\text { document et expliciter les critères de correction. }\end{array}$ & $\begin{array}{l}\text { 1. Individuellement, poursuivre la réflexion amorcée au cours 6. Ce } \\
\text { texte, qui doit être remis à la fin de la période, fera l'objet d'une évalua- } \\
\text { tion sommative. Les élèves disposent de deux périodes. }\end{array}$ \\
\hline
\end{tabular}

\section{Cours 9 : Retour sur le commentaire critique sur Nikolski (cours facultatif)}

\begin{tabular}{|c|c|}
\hline Pratique & Questionnement réciproque \\
\hline Composante de la lecture littéraire sollicitée & Compréhension, interprétation \\
\hline TÂCHES DE L'ENSEIGNANT & TÂCHES DES ÉLÈVES \\
\hline $\begin{array}{l}\text { 1. Collectivement, interroger les élèves : D’après eux, « Nikolski» mérite- } \\
\text { t-il des prix ou non? Pourquoi? Puis, mentionner les cinq prix reçus par } \\
\text { Nikolski : le Prix des libraires du Québec } 15^{e} \text { anniversaire (2008), le Prix } \\
\text { des libraires du Québec (2006), le Prix des collégiens (2006), le Prix An- } \\
\text { ne-Hébert (2006) ainsi que le Prix Printemps des lecteurs-Lavinal (2007). } \\
\text { Leur opinion est-elle modifiée? Pourquoi? Ces prix ont-ils un impact, selon } \\
\text { eux, sur la valeur littéraire de Nikolski? Il importe de montrer aux élèves } \\
\text { que les opinions peuvent diverger... }\end{array}$ & $\begin{array}{l}\text { 1. Partager et expliciter leur opinion sur Nikolski, revoir leur point de vue } \\
\text { le cas échéant. }\end{array}$ \\
\hline
\end{tabular}

\section{Cours 10 : Étude de l'écriture de Nicolas Dickner (cours facultatif)}

\begin{tabular}{|l|l|}
\hline \multicolumn{1}{|c|}{ Pratique } & \multicolumn{1}{|c|}{ Questionnement réciproque, écriture d'invention } \\
\hline \multicolumn{1}{|c|}{ TÂCHES DE L'ENSEIGNANT } & \multicolumn{1}{c|}{ Compréhension, interprétation } \\
\hline \multicolumn{1}{|c|}{ TÂCHES DES ÉLÈVES } \\
\hline $\begin{array}{l}\text { 1. Présenter la tâche : rédiger une rédaction créative inspirée du roman. } \\
\text { Puis, distribuer le document « 10.1 Écriture d'invention » et examiner } \\
\text { avec les élèves les consignes et les critères de correction. }\end{array}$ & $\begin{array}{l}\text { 1. Prendre connaissance des consignes de la rédaction créative (docu- } \\
\text { ment 10.1). }\end{array}$ \\
\hline $\begin{array}{l}\text { 2. Collectivement, demander de faire ressortir les caractéristiques de } \\
\text { l'écriture de Dickner (allusions, présences de différents types de textes, } \\
\text { relais de narrations, narrateurs différents, temps des verbes...). Il est } \\
\text { important de bien expliciter chacune des caractéristiques et de référer } \\
\text { les élèves aux notes de cours prises lors des mini-leçons. }\end{array}$ & $\begin{array}{l}\text { 2. Relever les caractéristiques de l'écriture de Dickner, avec l'aide de } \\
\text { l'enseignant, et les noter sur le document 10.1. }\end{array}$ \\
\hline $\begin{array}{l}\text { 3. Inviter les élèves à effectuer individuellement l'étape du remue-mé- } \\
\text { ninge. }\end{array}$ & $\begin{array}{l}\text { 3. Individuellement, effectuer l'étape du remue-méninge sur le docu- } \\
\text { ment 10.1. }\end{array}$ \\
\hline $\begin{array}{l}\text { 4. S'il dispose d'assez de temps, demander de se regrouper en équipe } \\
\text { de quatre élèves et de mettre en commun les idées afin de les appro- } \\
\text { fondir, les nuancer. }\end{array}$ & 4. En équipe de quatre, échanger les idées. Les améliorer, les modifier. \\
\hline
\end{tabular}

\section{Cours 11 et 12 : Rédaction créative sur Nikolski (cours facultatif)}

\begin{tabular}{|c|c|}
\hline Pratique & Écriture d'invention \\
\hline Composante de la lecture littéraire sollicitée & Compréhension, interprétation \\
\hline TÂCHES DE L'ENSEIGNANT & TÂCHES DES ÉLÈVES \\
\hline $\begin{array}{l}\text { 1. Réserver cette période pour poursuivre la rédaction. Circuler et } \\
\text { apporter son aide, le cas échéant. }\end{array}$ & $\begin{array}{l}\text { 1. Individuellement, poursuivre la rédaction du brouillon (document } \\
10.1 \text { remis au cours 10) et corriger le texte (contenu et langue). }\end{array}$ \\
\hline
\end{tabular}




\section{Cours 13 : Retour sur les différents textes rédigés et sur l'expérience de lecture (cours facultatif)}

\begin{tabular}{|c|c|}
\hline Pratique & Écriture d'invention, questionnement réciproque \\
\hline Composante de la lecture littéraire sollicitée & Compréhension, interprétation \\
\hline TÂCHES DE L'ENSEIGNANT & TÂCHES DES ÉLÈVES \\
\hline $\begin{array}{l}\text { 1. Lecture, par les élèves ou par l'enseignant, de quelques rédactions } \\
\text { créatives transposées sur transparent ou photocopiées. Pour chacun } \\
\text { des textes lus, questionner les élèves: }\end{array}$ & $\begin{array}{l}\text { 1. Prendre connaissance des textes écrits par leurs camarades. Réfléchir } \\
\text { aux effets littéraires de chacun des textes et aux « retombées », aux « } \\
\text { effets » de l'écriture d'invention. }\end{array}$ \\
\hline \multicolumn{2}{|l|}{$\begin{array}{l}\text { - Repérez les effets littéraires: En quoi ce qu'a écrit l'auteur de ce texte } \\
\text { est-il littéraire? }\end{array}$} \\
\hline $\begin{array}{l}\text { - En quoi l'écriture d'invention permet-elle de mieux effectuer une lecture } \\
\text { littéraire, c'est-à-dire en quoi permet-elle de mieux comprendre et inter- } \\
\text { préter le roman? Ceci peut être fait collectivement ou en équipe. }\end{array}$ & \\
\hline
\end{tabular}

